\title{
GCU
}

Glasgow Caledonian

University

University for the Common Good

\section{Biogeography-based optimization with covariance matrix based migration}

Chen, Xu; Tianfield, Huaglory; Du, Wenli; Liu, Guohai

Published in:

Applied Soft Computing

DOI:

10.1016/j.asoc.2016.04.022

Publication date:

2016

Document Version

Author accepted manuscript

Link to publication in ResearchOnline

Citation for published version (Harvard):

Chen, X, Tianfield, H, Du, W \& Liu, G 2016, 'Biogeography-based optimization with covariance matrix based migration', Applied Soft Computing, vol. 45, pp. 71-85. https://doi.org/10.1016/j.asoc.2016.04.022

\section{General rights}

Copyright and moral rights for the publications made accessible in the public portal are retained by the authors and/or other copyright owners and it is a condition of accessing publications that users recognise and abide by the legal requirements associated with these rights.

Take down policy

If you believe that this document breaches copyright please view our takedown policy at https://edshare.gcu.ac.uk/id/eprint/5179 for details of how to contact us. 


\title{
Biogeography-based Optimization with Covariance Matrix based Migration
}

\author{
Xu Chen ${ }^{\text {a, }}$, Huaglory Tianfield ${ }^{\mathrm{b}}$, Wenli Duc ${ }^{\mathrm{c}}$, Guohai Liu ${ }^{\mathrm{a}}$ \\ ${ }^{a}$ School of Electrical and Information Engineering, Jiangsu University, Zhenjiang 212013, China \\ ${ }^{b}$ School of Engineering and Built Environment, Glasgow Caledonian University, Glasgow G4 OBA, UK \\ ${ }^{c}$ Key Laboratory of Advanced Control and Optimization for Chemical Processes, Ministry of Education, East \\ China University of Science and Technology, Shanghai, 200237, China \\ Email:xuchen@ujs.edu.cn, h.tianfield@gcu.ac.uk,wldu@ecust.edu.cn, ghliu@ujs.edu.cn \\ * Corresponding authors: Xu Chen
}

\begin{abstract}
Biogeography-based optimization (BBO) is a new evolutionary algorithm. The major problem of basic BBO is that its migration operator is rotationally variant, which leaves BBO performing poorly in non-separable problems. To overcome this drawback of $\mathrm{BBO}$, in this paper, we propose the covariance matrix based migration (CMM) to relieve BBO's dependence upon the coordinate system so that BBO's rotational invariance is enhanced. By embedding the CMM into $\mathrm{BBO}$, we put forward a new BBO approach, namely biogeography-based optimization with covariance matrix based migration, called CMM-BBO. Specifically, CMM-BBO algorithms are developed by the CMM operator being randomly combined with the original migration in various existing BBO variants. Numeric simulations on 37 benchmark functions show that our CMM-BBO approach effectively improves the performance of the existing $\mathrm{BBO}$ algorithms.
\end{abstract}

Keyword: biogeography-based optimization; covariance matrix based migration; global numeric optimization; evolutionary algorithm; rotational invariance

\section{Introduction}

Inspired from the nature, a variety of evolutionary algorithms (EAs) has been developed to effectively tackle global optimization problems, for example, genetic algorithms (GA)[1], evolution strategies (ES)[2], differential evolution (DE)[3-5], particle swarm optimization (PSO) $[6,7]$ and so on. EAs have the advantages such as robustness, reliability, global search capability and little or no prior knowledge required.

Biogeography-based optimization (BBO), proposed by Simon[8], is a new EA based on biogeographic evolution. $\mathrm{BBO}$ has proven itself a competitive heuristic to other EAs on a wide range of problems [8-12].

To improve the performance of basic $\mathrm{BBO}$, a number of $\mathrm{BBO}$ variants have been proposed, which generally fall into three categories, i.e., (i) $\mathrm{BBO}$ with new or multiple migration or mutation operators, (ii) BBO hybrid with other EAs, and (iii) $\mathrm{BBO}$ with multiple populations or local topologies.

BBO with new or multiple migration or mutation operators: Gong et al. [13] proposed a real-coded BBO (called rcBBO) with three kinds of mutation operators, namely Gaussian mutation, Cauchy mutation, and Lévy mutation. Li and Yin [14] proposed a multi-operator BBO (called moBBO) with generalized migration operator using multi-parent migration model. Xiong et al.[15] proposed a $\mathrm{BBO}$ with polyphyletic migration operator and orthogonal learning strategy, called polBBO. Li et al.[16] proposed a perturbation optimization based BBO (called pBBO) with perturbation migration operator using sinusoidal migration model. Ma and Simon [17] proposed a blended BBO, for constrained optimization, with blended migration operator by analogue to the blended crossover operator in GA. Simon et al. [18] proposed a BBO with linearized migration that makes the migration more rotationally invariant.

BBO hybrid with other EAs: Du et al. [19] incorporated the elitism mechanism of evolutionary strategy and a new immigration refusal scheme into $\mathrm{BBO}$ and proposed a $\mathrm{BBO} / \mathrm{ES} / \mathrm{RE}$ algorithm. Gong et al. [20] incorporated DE's mutation operator with BBO's 
migration operator and proposed a DE/BBO algorithm, taking advantage of BBO's exploitation ability and DE's exploration ability. Boussaid et al. [21] incorporated DE with BBO through a two-stage updating mechanism and proposed a DE hybrid BBO algorithm. Kundra and Sood [22] combined PSO with BBO to optimize shortest path problems. Savsani et al. [23] incoprporated artificial immune algorithm and ant colony optimization with BBO and proposed four hybrid BBO variants.

BBO with multiple populations or local topologies: Zheng et al. [24] integrated three different local topologies (i.e., ring, square, and random) in BBO to enhance BBO's exploration ability, and proposed a localized BBO. Zheng et al. [25] divided the whole population into multiple sub-populations with each sub-population being evolved through a separate $\mathrm{BBO}$, and proposed a cooperative coevolutionary biogeography-based optimizer (called cBBO). Ma et al. [26] proposed a BBO with an ensemble of migration models using three parallel populations, each implementing a different migration model.

In addition to the three categories of BBO variants above, Ergeze et al. [27] proposed an oppositional BBO using opposition-based learning. Saremi et al. [28] proposed a chaotic BBO using ten chaotic maps to define selection, emigration, and mutation probabilities.

In $\mathrm{BBO}$ algorithms as mentioned above, either basic $\mathrm{BBO}$ or variants, the migration operator is crucial. In fact, it is through the migration operator that multiple parents contribute towards generating an offspring. However, the migration operators in the existing $\mathrm{BBO}$ algorithms are heavily dependent upon the coordinate systems, which leaves poor performance in dealing with non-separable problems[18]. A non-separable problem is one the fitness of which depends upon the variables combinatorially rather than individually. In other words, variables in a non-separable problem are tightly intermeshed with one another.

Simon et al pointed out [18] that a major drawback of BBO is that it treats each solution feature independently, which leaves BBO rotationally variant. Rotational variance means that BBO generally performs poorly when applied to non-separable problems. However, most real-world problems are non-separable. Thus, rotational variance restricts BBO's applicability to wider problems.

To address this drawback of $\mathrm{BBO}$, the key question is: how to relieve BBO's dependence upon the coordinate system and enhance BBO's rotational invariance?

Covariance Matrix Learning (CML) was first adopted in covariance matrix adaptation evolution strategy (CMAES) [2]. CML effectively adapts the search according to the landscape of the optimization function. Basically, CML rotates the coordinate system to make the problem pseudo-separable. CML employed in DE makes the crossover rotationally invariant [29, 30], which significantly improves the performance of DE.

In this paper we will propose the covariance matrix based migration (CMM) to relieve BBO's dependence upon the coordinate system so that BBO's rotational invariance is enhanced. By use of our proposed CMM operator, the original coordinate system is rotated into an eigenvectorbased one, in which solutions can share their information more efficiently.

By embedding the CMM into $\mathrm{BBO}$, we put forward a new $\mathrm{BBO}$ approach, namely biogeography-based optimization with covariance matrix based migration, called CMM-BBO. Specifically, CMM-BBO algorithms are developed by the CMM operator being randomly combined with the original migration in various exsiting $\mathrm{BBO}$ algorithms.

The remainder of the paper is arranged as follows. Section 2 proposes the covariance matrix based migration and puts forward the CMM-BBO approach. Section 3 conducts thorough performance evaluations of four CMM-BBO algorithms through numeric simulations on 37 benchmark functions and comparisons with other EAs. Lastly, Section 4 draws the conclusions.

\section{BBO with Covariance Matrix Based Migration}

\subsection{Preliminary: Basic BBO}

$\mathrm{BBO}[8]$ is a new population-based, biogeographically inspired global optimization algorithm. In BBO, each individual is regarded as a "habitat" or "island" with a Habitat Suitability Index (HSI), which is similar to the fitness in EAs. A good solution means a habitat with a high HSI, while a poor solution indicates a habitat with a low HSI.

A solution can be represented by a set of Suitability Index Variables (SIV). In BBO's 
migration process, high HSI solutions should share their features with low HSI ones; while low HIS solutions take in new features from high HIS ones. In BBO, each individual has its own immigration rate $\lambda$ and emigration rate $\mu$, which can be calculated based on HSI. A high HSI habitat has a high species emigration rate $\mu$ while a low HSI habitat has a high species immigration rate $\lambda$. For example, in a linear model of species richness, a habitat $H_{i}$ 's immigration rate $\lambda_{i}$ and emigration rate $\mu_{i}$ can be calculated as follows.

$$
\begin{aligned}
\lambda_{i} & =I\left(1-\frac{i}{n}\right) \\
\mu_{i} & =E\left(\frac{i}{n}\right)
\end{aligned}
$$

where $I$ is the maximum immigration rate, $E$ the maximum emigration rate, $n$ the population size, $i$ the index of the individual in order, where $i=1$ denoting the worst individual while $i=n$ denoting the best. Eq. (1) and (2) are called linear migration model of the migration rates.

Migration modifies habitats by mixing the features within a population. BBO also uses a mutation operator to change the SIV of a habitat itself, and thus increases the diversity of a population. For each habitat $H_{i}$, species count probability $P_{i}$, computed from $\lambda_{i}$ and $\mu_{i}$, measures the a priori likelihood that the habitat is expected to become a solution to the problem. In reality, either a very high HSI habitat or a very low HSI habitat is rarely probable, but most probable is a medium HSI habitat. A habitat's mutation rate $\pi_{i}$ is inversely proportional to its probability, i.e.,

$$
\pi_{i}=\pi_{\max }\left(1-\frac{P_{i}}{P_{\max }}\right)
$$

where $\pi_{\max }$ is a control parameter and $P_{\max }$ the maximum habitat probability in a population.

Basic $\mathrm{BBO}$ can be formulated as in Algorithm 1, where $D$ is the dimension of the optimization problem, $l_{d}$ and $u_{d}$ the lower and upper bounds of the $d$-th dimension, respectively, and rand a random number function uniformly distributed in $[0,1]$.

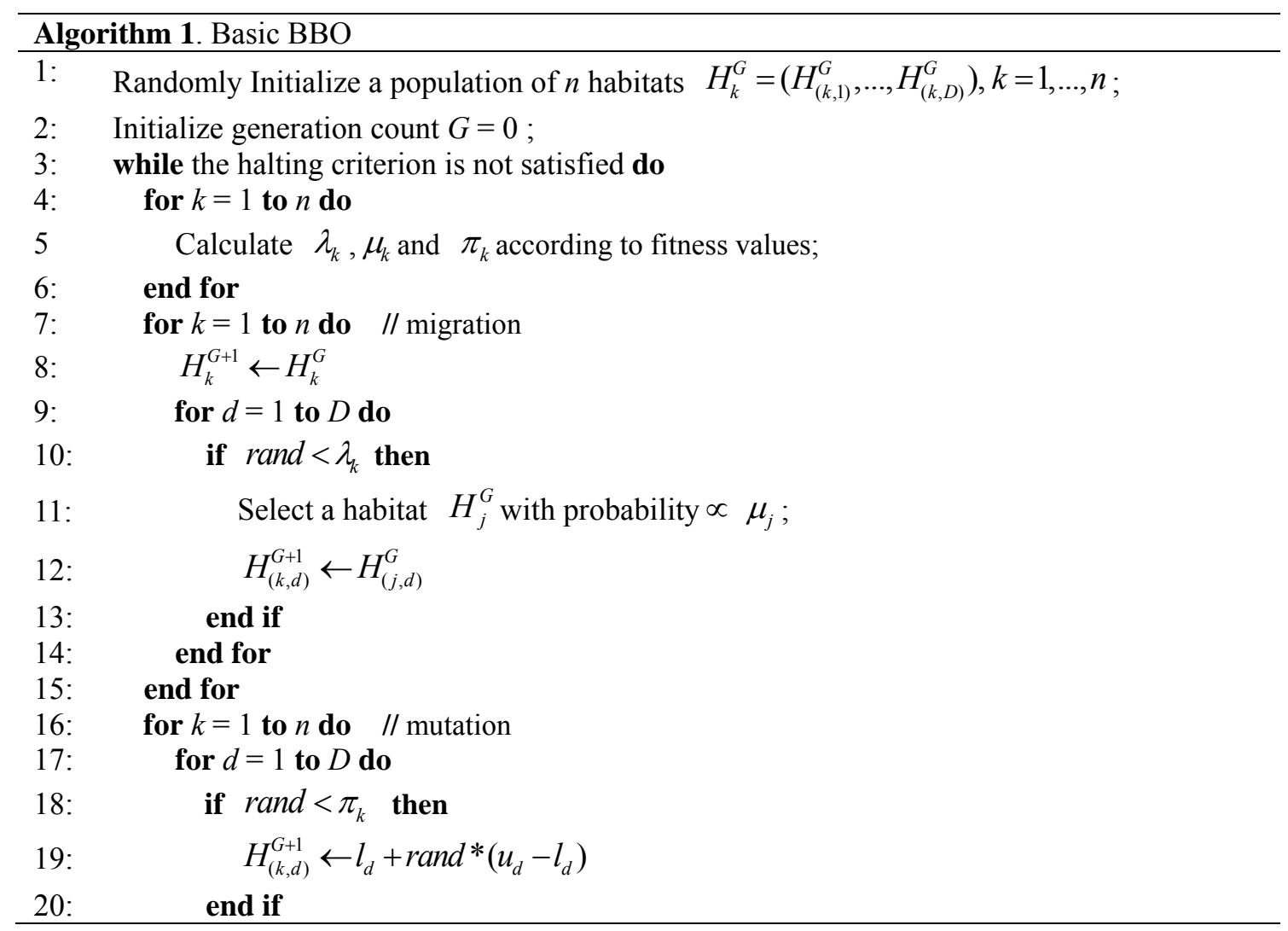




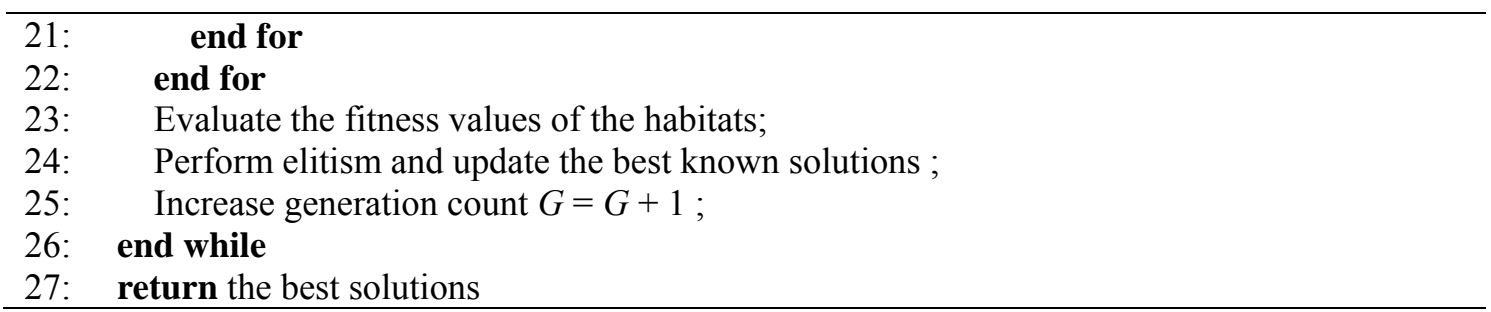

\subsection{Covariance Matrix Based Migration}

The core of the covariance matrix based migration is the original coordinate system being rotated into an eigenvectorbased one, in which habitants can share their information more efficiently. Fig. 1 illustrates in contour plots the original migration and the covariance matrix based migration, respectively. The migration for $\mathrm{BBO}$ can be carried out more efficiently in the eigenvectorbased coordinate system.

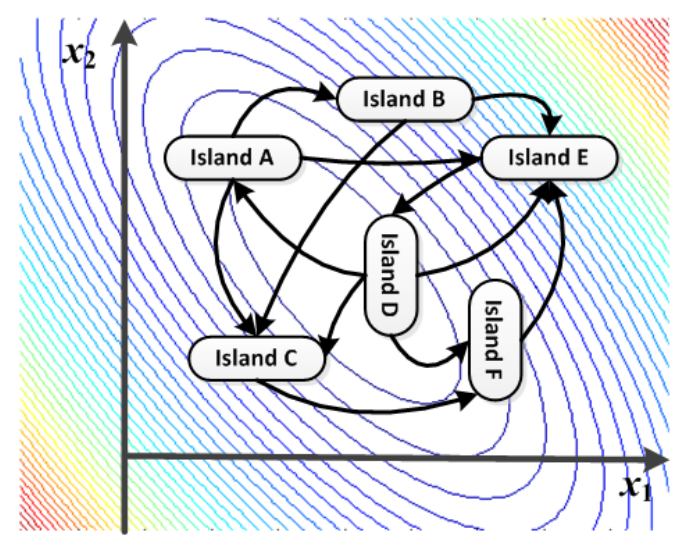

(a)

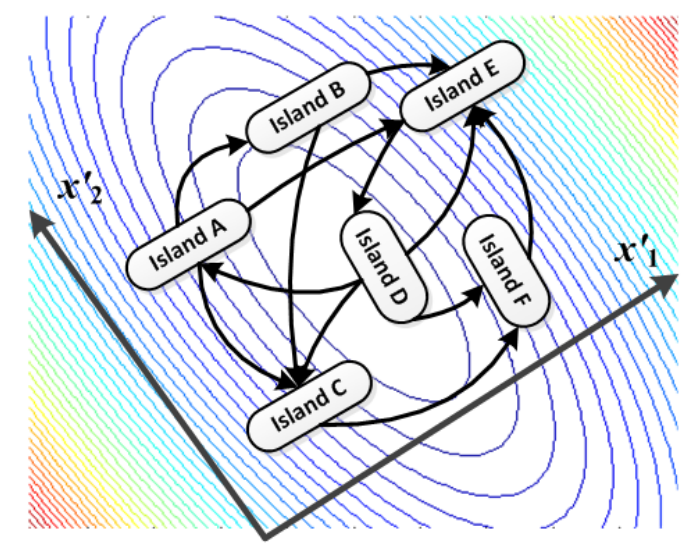

(b)

Fig. 1. (a) Original migration, (b) Covariance matrix based migration

Let's consider a population $\mathrm{H}$,

$$
\left.\begin{array}{l}
H=\left[H_{1}^{G}, \ldots, H_{k}^{G}, \ldots, H_{n}^{G}\right]^{T} \\
H_{k}^{G}=\left[H_{(k, 1)}^{G}, \ldots, H_{(k, j)}^{G}, \ldots, H_{(k, D)}^{G}\right]
\end{array}\right\} k=1, \ldots, n ; j=1, \ldots, D
$$

where $H$ is a $n \times D$ matrix, $n$ the population size, $D$ the number of independent variables, $G$ the generation count, and $H_{k}^{G}$ the habitant with index $k$.

The covariance between the $i$-th and the $j$-th dimensions of the population in the $G$-th generation is defined as below:

$$
\operatorname{cov}(i, j)=\frac{\sum_{k=1}^{n}\left(H_{(k, i)}^{G}-\bar{H}_{i}^{G}\right)\left(H_{(k, j)}^{G}-\bar{H}_{j}^{G}\right)}{n-1}, i=1, \ldots, D ; j=1, \ldots, D
$$

where $\bar{H}_{i}^{G}=\sum_{k=1}^{n} H_{(k, i)}^{G} / n$ denotes the means of the variables in the $i$-th dimension. The covariance matrix $\operatorname{Cov}(H)$ can be defined in terms of the covariance, i.e.,

$$
\operatorname{Cov}(H)=\left[c_{i j}\right]_{D \times D}=[\operatorname{cov}(i, j)]_{D \times D}
$$

In order to compute the eigenvectors, we factorize the covariance matrix $\operatorname{Cov}(H)$ into its canonical form, i.e.,

$$
\operatorname{Cov}(H)=Q_{H} \Lambda_{H} Q_{H}{ }^{T}
$$

where $Q_{H}$ is the $D \times D$ matrix that has the eigenvector of $\operatorname{Cov}(H)$ as its $i$-th column, and $\Lambda_{H}$ the diagonal matrix that has the corresponding eigenvalues as its diagonal entries, respectively. 
Factorizing a matrix into its canonical form is called eigenvalue decomposition.

After the eigenvalue decomposition, the habitant in the original coordinate system can be rotated into the eigenvector-based one as follows.

$$
\left.\begin{array}{l}
e i g H_{k}^{G}=H_{k}^{G} * Q_{H} \\
e i g H_{k}^{G}=\left(e i g H_{(k, 1)}^{G}, \ldots, e i g H_{(k, j)}^{G}, \ldots, e i g H_{(k, D)}^{G}\right)
\end{array}\right\} k=1, \ldots, n ; j=1, \ldots, D
$$

where $e i g H_{k}^{G}$ denotes the rotated habitant, and $e i g H_{(k, j)}^{G}$ the rotated SIV in the eigenvector-based coordinate system.

Let's call the migration in the eigenvectorbased coordinate system as covariance matrix based migration. Now, we can perform the BBO migration in the eigenvector-based coordinate system.

Let's denote the new migrated habitant after applying the covariance matrix based migration as $e i g H_{k}^{G+1}$. It can then be rotated back into the original coordinate system as follows.

$$
H_{k}^{G+1}=e i g H_{k}^{G+1} * Q_{H}^{T}
$$

To sum up, the covariance matrix based migration (CMM) consists of the eigenvalue decompostition based rotation of the original coordinate system and the migration in the eigenvector-based coordinate system. CMM can be formulated as in Algorithm 2, called CMM operator.

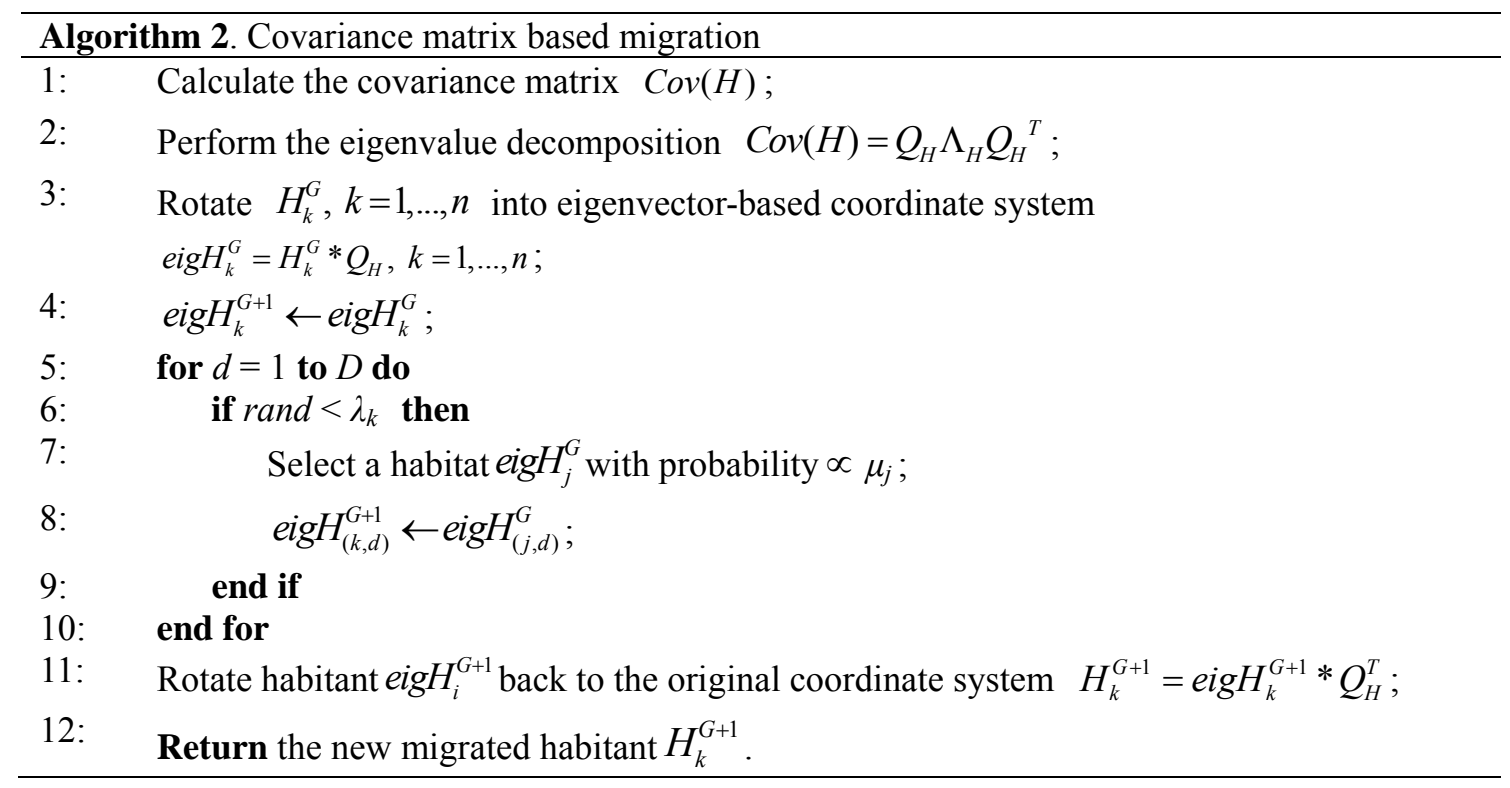

\subsection{CMM-BBO Algorithms}

By embedding the covariance matrix based migration into BBO, we can put forward a new $\mathrm{BBO}$ approach, namely biogeography-based optimization with covariance matrix based migration, called CMM-BBO. In particular, to prevent ineffective behavior caused by the rotational variance, we devise a parameter $P_{e}$ in CMM-BBO to control the ratio of CMM to the original migration. The algorithmic structure of CMM-BBO can be illustrated in Fig. 2. Since the switch is based on a random number, i.e., whether rand $<P_{e}$ or not, the covariance matrix based migration is being randomly combined with the original migration.

It should be pointed out that the $\mathrm{BBO}$ in $\mathrm{CMM}-\mathrm{BBO}$ can be either the basic $\mathrm{BBO}$ algorithm or any $\mathrm{BBO}$ variant algorithm, and whatever $\mathrm{BBO}$ algorithm is used, the framework of CMM-BBO remains the same.Furthermore, normally, $0<P_{e}<1$. There are two extreme cases. When $P_{e}=1$, CMM-BBO only uses the covariance matrix based migration to generate offspring. When $P_{e}=0, \mathrm{CMM}-\mathrm{BBO}$ reverts to the basic $\mathrm{BBO}$ with the original migration. In this sense, our proposed CMM-BBO approach also serves as a unified framework between basic $\mathrm{BBO}$ and $\mathrm{BBO}$ variant algorithms. 


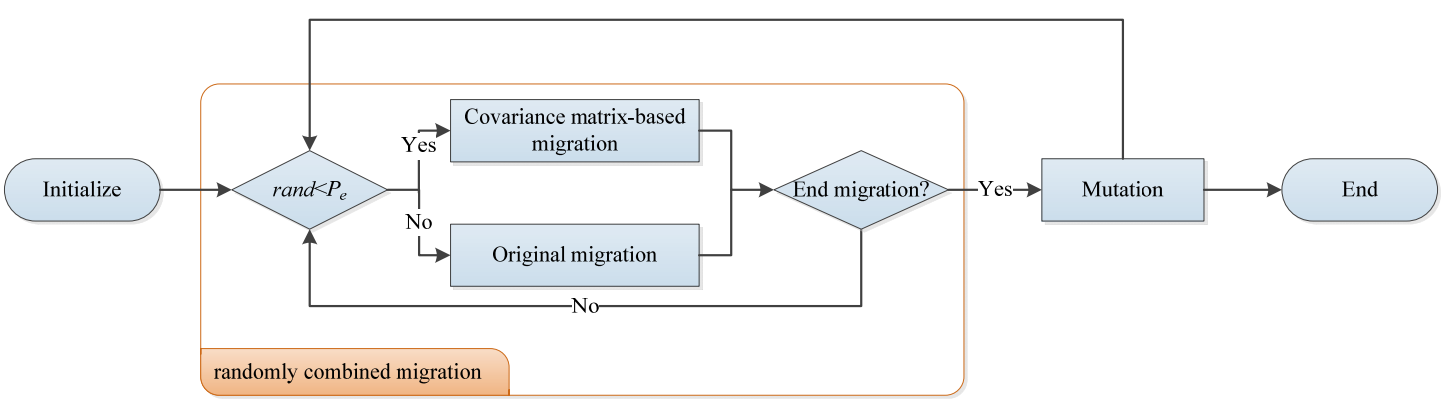

Fig. 2. Framework of CMM-BBO

CMM-BBO can be formulated as in Algorithm 3. Various CMM-BBO algorithms can be developed by the CMM operator being randomly combined with the original migration in various $\mathrm{BBO}$ algrothims. In this paper, we have selected four existing representative $\mathrm{BBO}$ variants, namely, basic real-code $\mathrm{BBO}(\mathrm{rcBBO})$ [13], real-code $\mathrm{BBO}$ with Gaussian mutation (rcBBOg) [13], perturbation based $\mathrm{BBO}$ with Gaussian mutation (pBBO)[16], and $\mathrm{BBO}$ hybrid with $\mathrm{DE}$ (DE/BBO)[20]. The CMM-BBO algorithms correspondingly developed are denoted as CMM-rcBBO, CMM-rcBBOg, CMM-pBBO, and CMM-DE/BBO, respectively.

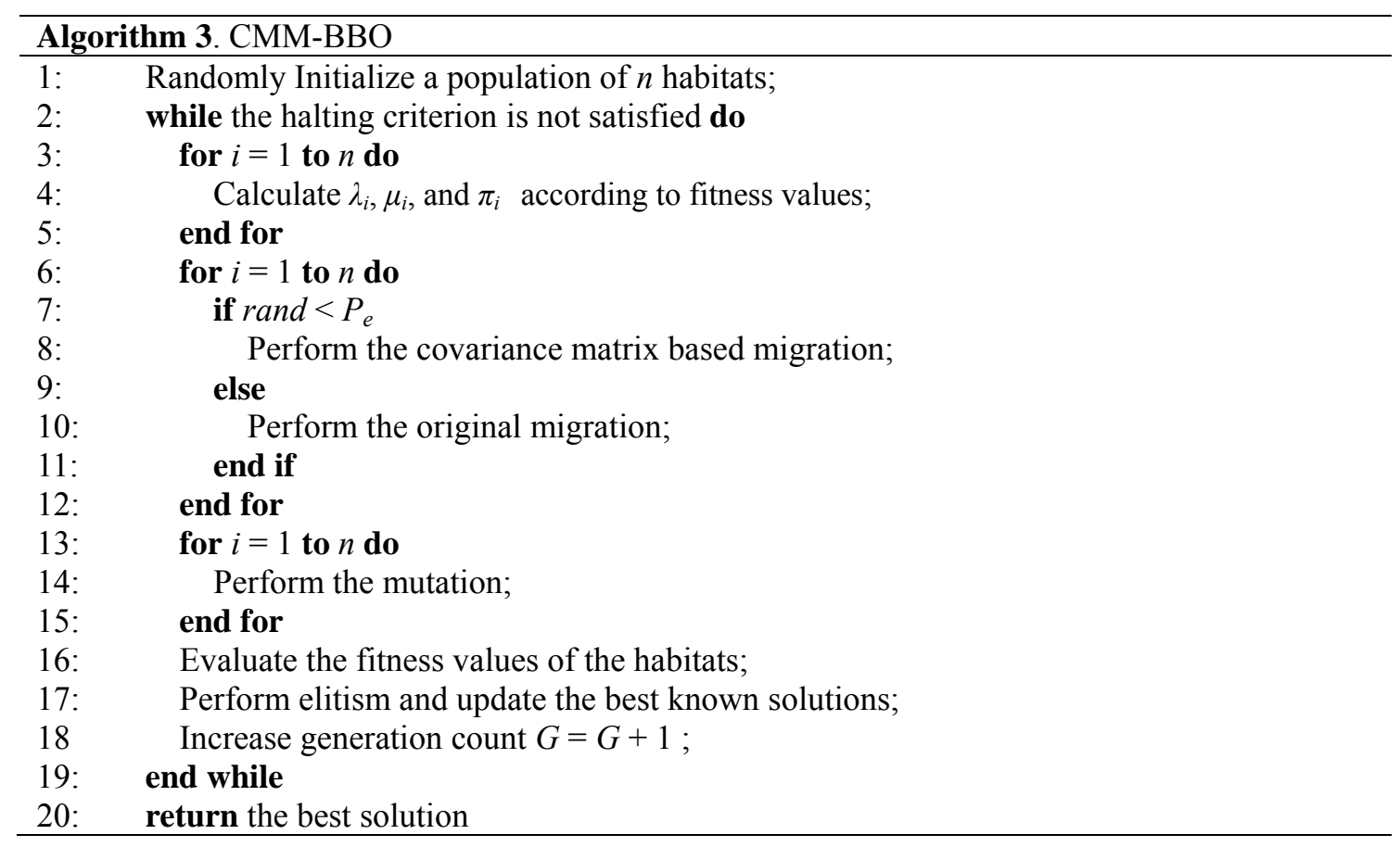

\section{Performance Evaluations}

To conduct the performance evaluations, we employ 37 benchmark functions, as listed in Table 1. The first 23 functions, f01 f23, are the same as in Yao et al. [31], while the rest 14 functions, F01 F14, from the same as in CEC2005 [32]. Functions f01 f04 and F01 F05 are unimodal, while the rest 28 functions are multimodal, in particular, F06 F12 are basic multimodal functions, and F13 F14 expanded multimodal functions. Functions f01 f04 are high-dimensional unimodal functions. Function f05 is a multimodal function when $D>3$. Function $\mathrm{f} 6$ is a high-dimensional discontinuous step function with one minimum. Function $\mathrm{f} 7$ is a high-dimensional function with noisy perturbation. Functions $\mathrm{f} 8 \sim \mathrm{fl} 3$ are high-dimensional multimodal functions where the number of local minima grows exponentially with increased dimensions. Functions f14 $\sim$ f23 are low-dimensional functions with only a few local minima. Among all the functions, 9 of them, i.e., f01, f06 f11, F01 and F09, are separable, while the rest 28 non-separable. 
Table 1. Benchmark functions for the numeric simulations

\begin{tabular}{|c|c|c|c|c|c|c|}
\hline & Name & & Dimension & Search space & Optima & Max_FEs \\
\hline f01 & Sphere model & separable & 30 & {$[-100,100]^{\mathrm{D}}$} & 0 & $1.50 \mathrm{E}+05$ \\
\hline $\mathrm{f} 02$ & Schwefel's problem 2.22 & non-separable & 30 & {$[-10,10]^{\mathrm{D}}$} & 0 & $2.00 \mathrm{E}+05$ \\
\hline f03 & Schwefel's problem 1.2 & non-separable & 30 & {$[-100,100]^{\mathrm{D}}$} & 0 & $5.00 \mathrm{E}+05$ \\
\hline f04 & Schwefel's problem 2.21 & non-separable & 30 & {$[-100,100]^{\mathrm{D}}$} & 0 & $5.00 \mathrm{E}+05$ \\
\hline f05 & Generalized Rosenbrock's functions & non-separable & 30 & {$[-30,30]^{\mathrm{D}}$} & 0 & $5.00 \mathrm{E}+05$ \\
\hline f06 & Step function & separable & 30 & {$[-100,100]^{\mathrm{D}}$} & 0 & $1.50 \mathrm{E}+05$ \\
\hline f07 & Quartic function & separable & 30 & {$[-1.28,1.28]^{\mathrm{D}}$} & 0 & $3.00 \mathrm{E}+05$ \\
\hline f08 & Generalized Schwefel's problem 2.26 & separable & 30 & {$[-500,500]^{\mathrm{D}}$} & -12569.5 & $3.00 \mathrm{E}+05$ \\
\hline f09 & Generalized Rastrigin's function & separable & 30 & {$[-5.12,5.12]^{\mathrm{D}}$} & 0 & $3.00 \mathrm{E}+05$ \\
\hline f10 & Ackley's function & separable & 30 & {$[-32,32]^{\mathrm{D}}$} & 0 & $1.50 \mathrm{E}+05$ \\
\hline f11 & Generalized Griewank function & separable & 30 & {$[-600,600]^{\mathrm{D}}$} & 0 & $2.00 \mathrm{E}+05$ \\
\hline $\mathrm{f} 12$ & Generalized Penalized function 1 & non-separable & 30 & {$[-50,50]^{\mathrm{D}}$} & 0 & $1.50 \mathrm{E}+05$ \\
\hline f13 & Generalized Penalized function 2 & non-separable & 30 & {$[-50,50]^{\mathrm{D}}$} & 0 & $1.50 \mathrm{E}+05$ \\
\hline f14 & Shekel's Foxholes function & non-separable & 2 & {$[-65.536,65.536]^{\mathrm{D}}$} & 0.99800383779445 & $1.00 \mathrm{E}+04$ \\
\hline f15 & Kowalik's function & non-separable & 4 & {$[-5,5]^{\mathrm{D}}$} & 0.0003075 & $4.00 \mathrm{E}+05$ \\
\hline f16 & Six-Hump Camel-Back function & non-separable & 2 & {$[-5,5]^{\mathrm{D}}$} & -1.03162845348988 & $1.00 \mathrm{E}+04$ \\
\hline f17 & Branin Function & non-separable & 2 & {$[-5,10] *[0,15]$} & 0.397887357729738 & $1.00 \mathrm{E}+04$ \\
\hline f18 & Glodstein-Price function & non-separable & 2 & {$[-2,2]^{\mathrm{D}}$} & 2.999999999999992 & $1.00 \mathrm{E}+04$ \\
\hline f19 & Hartman's function 1 & non-separable & 3 & {$[0,1]^{\mathrm{D}}$} & -3.86278214782076 & $1.00 \mathrm{E}+04$ \\
\hline $\mathrm{f} 20$ & Hartman's function 2 & non-separable & 6 & {$[0,1]^{\mathrm{D}}$} & -3.32199517158424 & $2.00 \mathrm{E}+04$ \\
\hline $\mathrm{f} 21$ & Shekel's Function 1 & non-separable & 4 & {$[-0,10]^{\mathrm{D}}$} & -10.153199679 & $1.00 \mathrm{E}+04$ \\
\hline $\mathrm{f} 22$ & Shekel's Function 2 & non-separable & 4 & {$[-0,10]^{\mathrm{D}}$} & -10.4029405667869 & $1.00 \mathrm{E}+04$ \\
\hline $\mathrm{f} 23$ & Shekel's Function 3 & non-separable & 4 & {$[-0,10]^{\mathrm{D}}$} & -10.5364 & $1.00 \mathrm{E}+04$ \\
\hline F01 & Shifted sphere & separable & 30 & {$[-100,100]^{\mathrm{D}}$} & -450 & $3.00 \mathrm{E}+05$ \\
\hline F02 & Shifted Schwefel's problem 1.2 & non-separable & 30 & {$[-100,100]^{\mathrm{D}}$} & -450 & $3.00 \mathrm{E}+05$ \\
\hline F03 & Shifted rotated high conditioned elliptic & non-separable & 30 & {$[-100,100]^{\mathrm{D}}$} & -450 & $3.00 \mathrm{E}+05$ \\
\hline F04 & Shifted Schwefel's problem 1.2 & non-separable & 30 & {$[-100,100]^{\mathrm{D}}$} & -450 & $3.00 \mathrm{E}+05$ \\
\hline F05 & Schwefel's problem 2.6 & non-separable & 30 & {$[-100,100]^{\mathrm{D}}$} & -310 & $3.00 \mathrm{E}+05$ \\
\hline F06 & Shifted Rosenbrock & non-separable & 30 & {$[-100,100]^{\mathrm{D}}$} & 390 & $3.00 \mathrm{E}+05$ \\
\hline F07 & Shifted rotated Griewank's function & non-separable & 30 & {$[0,600]^{\mathrm{D}}$} & -180 & $3.00 \mathrm{E}+05$ \\
\hline F08 & Shifted rotated Ackley's function & non-separable & 30 & {$[-32,32]^{\mathrm{D}}$} & -140 & $3.00 \mathrm{E}+05$ \\
\hline F09 & Shifted Rastrigin & separable & 30 & {$[-100,100]^{\mathrm{D}}$} & -330 & $3.00 \mathrm{E}+05$ \\
\hline F10 & Shifted rotated Rastrigin & non-separable & 30 & {$[-5,5]^{\mathrm{D}}$} & -330 & $3.00 \mathrm{E}+05$ \\
\hline F11 & Shifted rotated weierstrass & non-separable & 30 & {$[-0.5,0.5]^{\mathrm{D}}$} & 90 & $3.00 \mathrm{E}+05$ \\
\hline F12 & Schwefel's problem 2.13 & non-separable & 30 & {$[-\pi, \pi]^{\mathrm{D}}$} & -590 & $3.00 \mathrm{E}+05$ \\
\hline F13 & Expanded extended F8 plus F2 & non-separable & 30 & {$[-3,1]^{\mathrm{D}}$} & -130 & $3.00 \mathrm{E}+05$ \\
\hline F14 & Rotated expanded extended Scaffe's F6 & non-separable & 30 & {$[-100,100]^{\mathrm{D}}$} & -300 & $3.00 \mathrm{E}+05$ \\
\hline
\end{tabular}

Three performance criteria are adopted from [32] as follows.

- Error: The error of a solution $x$ is defined as $f(x)-f(x *)$, where $x *$ is the global minimum provided in [33]. The minimum error is recorded when the maximum number of functional evaluations (Max_FEs) is reached in 30 independent runs. The Max_FEs values for the 37 functions are set the same as in [20], [32]. The mean and standard deviation of the errors are calculated for analysis.

- SR (number of successful runs): The successful run of an algorithm manifests the ability of the algorithm to obtain an optimization result no worse than the required accuracy level (RAL) before the search is terminated by the Max_FEs condition. For functions f01 f06, f08 f23, and F01 F14, RAL $=10^{-8}$; for functions f07, RAL $=10^{-2}$ as in [20], [32].

- Convergence: The convergence shows the mean error of the best solution over the total runs, in the respective experiments.

The parameter settings of the four existing BBO variants are the same as in the literature, 
respectively, as presented in Table 2 .

Table 2. Parameter settings of the four existing BBO algorithms

\begin{tabular}{ll}
\hline Algorithm & Parameters \\
\hline rcBBO & $n=100, I=E=1, \pi_{\text {max }}=0.005$, elitism parameter $K=2$ \\
rcBBOg & $n=100, I=E=1, \pi_{\text {max }}=0.005, K=2$ \\
pBBO & $n=100, I=E=1, \pi_{\text {max }}=0.005, K=2$ \\
DE/BBO & $n=100, I=E=1, \pi_{\text {max }}=0.005, K=2$, scaling factor $F=\operatorname{rand}(0.1,1)$, crossover probability $C R=0.9$. \\
\hline
\end{tabular}

For all the four CMM-BBO algorithms, $P_{e}$ is set as 0.5 . This is based on the thorough sensitivity analysis we have conducted to determine a proper value for parameter $P_{e}$. For details, see Appendix A. It should be noted, though, that it is impractical to have a universal optimal setting of parameter $P_{e}$ as it basically depends upon the specific problems.

\subsection{Performances of CMM-BBO Algorithms}

Table 3 compares the errors between the existing $\mathrm{BBO}$ variants and the corresponding CMM-BBO algorithms on the 37 benchmark functions. For each pair of the existing BBO and its CMM-BBO, the better performance in terms of mean error is highlighted in boldface. A nonparametric statistical test, called Wilcoxon's rank-sum test, between the existing BBO and its CMM-BBO is conducted at a 5\% significance level, so as to see whether the results obtained with the better performing algorithm significantly exhibit superior performance. "+ ", "-", and "=" symbolize the performance of the CMM-BBO algorithm being better or worse than, and similar to that of the existing $\mathrm{BBO}$, respectively.

Compared with rcBBO, of the 37 benchmark functions, CMM-rcBBO achieves significantly better performances on 33 ( 9 separable and 24 non-separable), not statistically different performances on 3, and poorer performance on only one non-separable function (F12). Therefore, it is fair to say that the CMM operator is able to enhance BBO's capability on non-separable as well as separable functions.

Our proposed CMM operator has remarkably improved the performance of rcBBOg. Compared with rcBBOg, of the 37 benchmark functions, CMM-rcBBOg exhibits significantly better performances on 33 ( 7 separable and 26 non-separable), not statistically different performances on 3 functions ( 1 separable and 2 non-separable), and poorer performance on only one separable function $\mathrm{f} 8$. What is more, on most of the non-separable functions, CMM-rcBBOg outperforms rcBBOg. In one word, the benefit of the $\mathrm{CMM}$ operator for rcBBOg is apparent.

The CMM operator improves the performance of $\mathrm{pBBO}$ as well. Compared with $\mathrm{pBBO}$, of the 37 benchmark functions, CMM-pBBO exhibits significantly better performances on 25 functions (4 separable and 21 non-separable), not statistically different performances on 9 functions ( 3 separable and 6 non-separable) and poorer performances on only the rest 3 functions (2 separable and 1 non-separable).

The performance of $\mathrm{DE} / \mathrm{BBO}$ is substantially improved by the CMM operator. Compared with $\mathrm{DE} / \mathrm{BBO}$, of the 37 benchmark functions, CMM-DE/BBO achieves statistically significant improvements on 22 functions ( 3 separable and 19 non-separable), not statistically different performances on 10 functions (4 separable and 6 non-separable), and poorer performances on only 5 functions ( 2 separable and 3 non-separable).

Table 4 presents the SR values of all the existing $\mathrm{BBO}$ variants and the corresponding CMM-BBO algorithms on the 37 benchmark functions. "+ ", "-", and "=" symbolize the SR value of the CMM-BBO algorithm being better or worse than, and similar to that of the existing BBO variant, respectively. As shown in Table 5, the CMM operator has improved the performances of all the existing four BBO variants in terms of SR values on most of the benchmark functions. This manifests that the CMM-BBO algorithms are more effective than the existing $\mathrm{BBO}$ variants. 
Table 3. Comparison of the errors between the existing $\mathrm{BBO}$ variants and the corresponding CMM-BBO algorithms

\begin{tabular}{|c|c|c|c|c|c|c|c|c|c|c|c|}
\hline \multirow{2}{*}{$\begin{array}{l}\text { Algorithm } \\
\text { Function }\end{array}$} & & \multicolumn{3}{|c|}{$\mathrm{rcBBO}$} & \multicolumn{2}{|c|}{ CMM-rcBBO } & \multicolumn{3}{|c|}{$\mathrm{rcBBOg}$} & \multicolumn{2}{|c|}{ CMM-rcBBOg } \\
\hline & & mean & SD & & mean & SD & mean & SD & & mean & SD \\
\hline f01 & separable & $2.10 \mathrm{E}+00$ & 7.45E-01 & + & $4.49 \mathrm{E}-11$ & 2.53E-11 & $5.26 \mathrm{E}-04$ & $2.14 \mathrm{E}-04$ & + & $4.81 \mathrm{E}-15$ & 1.83E-15 \\
\hline $\mathrm{f} 02$ & non-separable & $3.92 \mathrm{E}-01$ & $5.59 \mathrm{E}-02$ & + & $6.90 \mathrm{E}-07$ & 1.67E-07 & $5.14 \mathrm{E}-02$ & $9.81 \mathrm{E}-03$ & + & 7.98E-08 & 2.23E-08 \\
\hline f03 & non-separable & $3.74 \mathrm{E}+03$ & $1.26 \mathrm{E}+03$ & + & $2.04 \mathrm{E}+00$ & $2.69 \mathrm{E}+00$ & $2.32 \mathrm{E}+01$ & $9.12 \mathrm{E}+00$ & + & $1.16 \mathrm{E}+00$ & $5.95 \mathrm{E}-01$ \\
\hline f04 & non-separable & $1.39 \mathrm{E}+00$ & $3.10 \mathrm{E}-01$ & + & 6.75E-03 & 2.07E-02 & $6.49 \mathrm{E}-02$ & $2.75 \mathrm{E}-02$ & + & 1.12E-02 & $5.38 \mathrm{E}-03$ \\
\hline $\mathrm{f} 05$ & non-separable & $1.19 \mathrm{E}+02$ & $3.89 \mathrm{E}+01$ & + & $3.73 E+01$ & $2.43 E+01$ & $9.31 \mathrm{E}+01$ & $1.34 \mathrm{E}+02$ & $=$ & $3.44 \mathrm{E}+01$ & $2.29 \mathrm{E}+01$ \\
\hline f06 & separable & $2.23 \mathrm{E}+00$ & $1.38 \mathrm{E}+00$ & + & $0.00 \mathrm{E}+00$ & $0.00 \mathrm{E}+00$ & $0.00 \mathrm{E}+00$ & $0.00 \mathrm{E}+00$ & $=$ & $0.00 \mathrm{E}+00$ & $0.00 \mathrm{E}+00$ \\
\hline $\mathrm{f} 07$ & separable & $5.49 \mathrm{E}-03$ & $2.84 \mathrm{E}-03$ & + & 2.05E-03 & 7.62E-04 & 4.87E-03 & $1.72 \mathrm{E}-03$ & + & 2.08E-03 & $6.38 \mathrm{E}-04$ \\
\hline f08 & separable & $1.52 \mathrm{E}+00$ & $6.66 \mathrm{E}-01$ & + & $1.34 \mathrm{E}-02$ & 3.75E-11 & $4.76 \mathrm{E}+02$ & $2.24 \mathrm{E}+02$ & - & $3.14 \mathrm{E}+03$ & $6.47 \mathrm{E}+02$ \\
\hline f09 & separable & $2.41 \mathrm{E}-01$ & $1.00 \mathrm{E}-01$ & + & 8.41E-12 & $6.16 \mathrm{E}-12$ & $1.44 \mathrm{E}-02$ & $7.82 \mathrm{E}-03$ & + & $1.22 \mathrm{E}-13$ & $5.96 \mathrm{E}-14$ \\
\hline $\mathrm{f} 10$ & separable & $6.05 \mathrm{E}-01$ & $1.44 \mathrm{E}-01$ & + & 1.49E-06 & 4.64E-07 & $1.53 \mathrm{E}-02$ & $2.86 \mathrm{E}-03$ & + & 4.35E-08 & 1.53E-08 \\
\hline $\mathrm{f} 11$ & separable & $8.12 \mathrm{E}-01$ & $1.02 \mathrm{E}-01$ & + & 2.47E-04 & 1.35E-03 & $2.99 \mathrm{E}-01$ & $2.65 \mathrm{E}-01$ & + & 2.05E-03 & $4.30 \mathrm{E}-03$ \\
\hline $\mathrm{f} 12$ & non-separable & $1.06 \mathrm{E}-02$ & $1.13 \mathrm{E}-02$ & + & 2.11E-13 & 1.33E-13 & $6.26 \mathrm{E}-01$ & 7.61E-01 & + & 4.03E-17 & 3.12E-17 \\
\hline f13 & non-separable & 1.09E-01 & $2.58 \mathrm{E}-02$ & + & $2.80 \mathrm{E}-12$ & $2.39 \mathrm{E}-12$ & $1.18 \mathrm{E}-04$ & $1.23 \mathrm{E}-04$ & + & 1.14E-15 & $9.92 \mathrm{E}-16$ \\
\hline f14 & non-separable & $1.43 \mathrm{E}+00$ & $2.47 \mathrm{E}+00$ & + & 7.08E-01 & $1.69 \mathrm{E}+00$ & $3.02 \mathrm{E}+00$ & $4.07 \mathrm{E}+00$ & + & $1.59 \mathrm{E}+00$ & $2.55 \mathrm{E}+00$ \\
\hline $\mathrm{f} 15$ & non-separable & $2.62 \mathrm{E}-03$ & 4.87E-03 & + & 8.33E-04 & $5.40 \mathrm{E}-04$ & $4.11 \mathrm{E}-03$ & $6.37 \mathrm{E}-03$ & + & $6.88 \mathrm{E}-04$ & $5.34 \mathrm{E}-04$ \\
\hline f16 & non-separable & $2.35 \mathrm{E}-02$ & $2.66 \mathrm{E}-02$ & + & 1.26E-04 & $3.00 \mathrm{E}-04$ & $1.13 \mathrm{E}-02$ & $2.24 \mathrm{E}-02$ & + & $1.84 \mathrm{E}-04$ & 6.43E-04 \\
\hline $\mathrm{f} 17$ & non-separable & $1.19 \mathrm{E}-02$ & $1.83 \mathrm{E}-02$ & + & 1.57E-03 & 2.71E-03 & $8.76 \mathrm{E}-03$ & $1.44 \mathrm{E}-02$ & + & $1.08 \mathrm{E}-03$ & 3.02E-03 \\
\hline $\mathrm{f} 18$ & non-separable & $2.12 \mathrm{E}+00$ & $5.50 \mathrm{E}+00$ & + & 2.27E-03 & 1.22E-02 & $1.34 \mathrm{E}+00$ & $2.99 \mathrm{E}+00$ & + & 2.13E-02 & 1.05E-01 \\
\hline f19 & non-separable & $7.51 \mathrm{E}-03$ & $9.87 \mathrm{E}-03$ & + & 1.03E-04 & $2.56 \mathrm{E}-04$ & $1.21 \mathrm{E}-02$ & $1.37 \mathrm{E}-02$ & + & 1.17E-04 & 4.07E-04 \\
\hline $\mathrm{f} 20$ & non-separable & 4.97E-02 & $5.75 \mathrm{E}-02$ & + & 3.17E-02 & 5.35E-02 & $5.02 \mathrm{E}-02$ & $6.09 \mathrm{E}-02$ & + & $1.98 \mathrm{E}-02$ & 4.51E-02 \\
\hline $\mathrm{f} 21$ & non-separable & $5.36 \mathrm{E}+00$ & $2.93 \mathrm{E}+00$ & + & $2.26 \mathrm{E}+00$ & $3.11 \mathrm{E}+00$ & $3.91 \mathrm{E}+00$ & $3.33 \mathrm{E}+00$ & + & $1.83 E+00$ & $3.14 \mathrm{E}+00$ \\
\hline $\mathrm{f} 22$ & non-separable & $4.50 \mathrm{E}+00$ & $2.67 \mathrm{E}+00$ & + & $1.43 \mathrm{E}+00$ & $2.92 \mathrm{E}+00$ & $3.07 \mathrm{E}+00$ & $3.13 \mathrm{E}+00$ & + & $1.32 \mathrm{E}+00$ & $2.71 \mathrm{E}+00$ \\
\hline $\mathrm{f} 23$ & non-separable & $4.91 \mathrm{E}+00$ & $2.53 \mathrm{E}+00$ & + & $1.01 \mathrm{E}+00$ & $2.62 \mathrm{E}+00$ & $3.51 \mathrm{E}+00$ & $3.19 \mathrm{E}+00$ & + & 9.73E-01 & $2.53 E+00$ \\
\hline F01 & separable & $5.71 \mathrm{E}-01$ & $2.10 \mathrm{E}-01$ & + & 3.59E-12 & 2.13E-12 & $8.94 \mathrm{E}-05$ & $4.18 \mathrm{E}-05$ & + & 4.73E-16 & $1.94 \mathrm{E}-16$ \\
\hline F02 & non-separable & $6.88 \mathrm{E}+03$ & $2.59 \mathrm{E}+03$ & + & $3.37 \mathrm{E}+02$ & $1.59 \mathrm{E}+02$ & $1.88 \mathrm{E}+02$ & $8.67 \mathrm{E}+01$ & + & $3.67 \mathrm{E}+01$ & $1.80 \mathrm{E}+01$ \\
\hline F03 & non-separable & $1.65 \mathrm{E}+07$ & $7.83 \mathrm{E}+06$ & + & $2.78 \mathrm{E}+06$ & $7.70 \mathrm{E}+05$ & $3.44 \mathrm{E}+06$ & $1.47 \mathrm{E}+06$ & + & $1.71 \mathrm{E}+06$ & $5.72 E+05$ \\
\hline F04 & non-separable & $1.67 \mathrm{E}+04$ & $6.05 \mathrm{E}+03$ & + & $1.94 E+03$ & $3.53 \mathrm{E}+02$ & $2.02 \mathrm{E}+04$ & $8.00 \mathrm{E}+03$ & + & $5.04 \mathrm{E}+03$ & $1.89 E+03$ \\
\hline F05 & non-separable & $6.23 \mathrm{E}+03$ & $1.15 \mathrm{E}+03$ & + & $4.54 \mathrm{E}+03$ & $4.43 E+02$ & $6.42 \mathrm{E}+03$ & $1.03 \mathrm{E}+03$ & + & $5.02 E+03$ & $8.49 E+02$ \\
\hline F06 & non-separable & $8.72 \mathrm{E}+02$ & $2.55 \mathrm{E}+03$ & + & $5.40 \mathrm{E}+02$ & $1.64 \mathrm{E}+03$ & $4.11 \mathrm{E}+03$ & $5.76 \mathrm{E}+03$ & + & $2.82 E+02$ & $3.93 E+02$ \\
\hline F07 & non-separable & $5.34 \mathrm{E}+03$ & $1.16 \mathrm{E}+02$ & + & $3.71 \mathrm{E}+02$ & $1.01 E+02$ & $2.24 \mathrm{E}+03$ & $7.71 \mathrm{E}+01$ & + & $2.50 \mathrm{E}+02$ & $1.01 \mathrm{E}+02$ \\
\hline F08 & non-separable & $2.09 \mathrm{E}+01$ & $1.04 \mathrm{E}-01$ & $=$ & $2.09 \mathrm{E}+01$ & $6.12 \mathrm{E}-02$ & $2.07 \mathrm{E}+01$ & $1.29 \mathrm{E}-01$ & + & $2.06 \mathrm{E}+01$ & $9.38 \mathrm{E}-02$ \\
\hline F09 & separable & $2.86 \mathrm{E}-01$ & $1.12 \mathrm{E}-01$ & + & $1.49 \mathrm{E}-11$ & 2.63E-11 & $1.76 \mathrm{E}-02$ & $8.89 \mathrm{E}-03$ & + & $1.08 \mathrm{E}-13$ & 6.07E-14 \\
\hline F10 & non-separable & $5.12 \mathrm{E}+01$ & $1.48 \mathrm{E}+01$ & $=$ & $4.70 \mathrm{E}+01$ & $1.72 \mathrm{E}+01$ & $5.92 \mathrm{E}+01$ & $2.03 \mathrm{E}+01$ & $=$ & $5.05 \mathrm{E}+01$ & $1.64 \mathrm{E}+01$ \\
\hline F11 & non-separable & $3.23 \mathrm{E}+01$ & $3.51 \mathrm{E}+00$ & + & $1.59 \mathrm{E}+01$ & $2.37 \mathrm{E}+00$ & $3.13 \mathrm{E}+01$ & $3.16 \mathrm{E}+00$ & + & $1.51 \mathrm{E}+01$ & $3.20 \mathrm{E}+00$ \\
\hline F12 & non-separable & $1.66 \mathrm{E}+00$ & $1.01 \mathrm{E}+00$ & - & $7.60 \mathrm{E}+03$ & $7.30 \mathrm{E}+03$ & $1.95 \mathrm{E}+04$ & $1.54 \mathrm{E}+04$ & + & $1.03 E+00$ & $1.13 E+00$ \\
\hline F13 & non-separable & $1.26 \mathrm{E}+00$ & $3.08 \mathrm{E}-01$ & $=$ & $1.14 \mathrm{E}+00$ & $1.80 \mathrm{E}-01$ & $1.24 \mathrm{E}+00$ & 1.44E-01 & + & $1.11 E+00$ & 2.17E-01 \\
\hline F14 & non-separable & $1.32 \mathrm{E}+01$ & $3.90 \mathrm{E}-01$ & + & $1.26 \mathrm{E}+01$ & 3.96E-01 & $1.36 \mathrm{E}+01$ & $2.59 \mathrm{E}-01$ & + & $1.30 \mathrm{E}+01$ & 5.16E-01 \\
\hline$+/=/-$ & & & & & & & & & & & \\
\hline
\end{tabular}


Table 3. (Continued)

\begin{tabular}{|c|c|c|c|c|c|c|c|c|c|c|c|}
\hline \multirow{2}{*}{$\begin{array}{c}\text { Algorithm. } \\
\text { Function }\end{array}$} & & \multicolumn{2}{|c|}{ pBBO } & & \multicolumn{2}{|c|}{ CMM-pBBO } & \multicolumn{2}{|c|}{$\mathrm{DE} / \mathrm{BBO}$} & & \multicolumn{2}{|c|}{ CMM-DE/BBO } \\
\hline & & mean & SD & & mean & SD & mean & SD & & mean & SD \\
\hline f01 & separable & 7.74E-08 & $3.28 \mathrm{E}-07$ & + & 6.31E-11 & 3.18E-11 & $9.92 \mathrm{E}-21$ & $5.31 \mathrm{E}-21$ & + & $2.90 \mathrm{E}-25$ & 1.59E-25 \\
\hline f02 & non-separable & 3.32E-05 & $9.24 \mathrm{E}-05$ & + & 7.98E-06 & 2.17E-06 & 2.15E-18 & $6.99 \mathrm{E}-19$ & - & $7.82 \mathrm{E}-17$ & $2.54 \mathrm{E}-17$ \\
\hline $\mathrm{f} 03$ & non-separable & $2.15 \mathrm{E}-01$ & $1.11 \mathrm{E}-01$ & + & 2.29E-07 & 1.39E-07 & $6.32 \mathrm{E}+02$ & $2.21 \mathrm{E}+02$ & + & 1.53E-23 & 4.13E-23 \\
\hline f04 & non-separable & 7.87E-03 & $2.36 \mathrm{E}-03$ & + & $7.50 \mathrm{E}-06$ & $1.40 \mathrm{E}-06$ & $1.71 \mathrm{E}-07$ & $5.25 \mathrm{E}-08$ & + & 2.14E-15 & 1.33E-15 \\
\hline f05 & non-separable & $4.55 \mathrm{E}+01$ & $9.26 \mathrm{E}+01$ & $=$ & $2.50 \mathrm{E}+01$ & $1.38 \mathrm{E}+01$ & $1.80 \mathrm{E}+01$ & $3.73 \mathrm{E}-01$ & + & 2.14E-01 & 5.12E-01 \\
\hline f06 & separable & $0.00 \mathrm{E}+00$ & $0.00 \mathrm{E}+00$ & $=$ & $0.00 \mathrm{E}+00$ & $0.00 \mathrm{E}+00$ & $0.00 \mathrm{E}+00$ & $0.00 \mathrm{E}+00$ & $=$ & $0.00 \mathrm{E}+00$ & $0.00 \mathrm{E}+00$ \\
\hline f07 & separable & 2.04E-03 & 7.00E-04 & - & $9.15 \mathrm{E}-03$ & $2.97 \mathrm{E}-03$ & $6.05 \mathrm{E}-03$ & $1.54 \mathrm{E}-03$ & + & 2.66E-03 & $7.46 \mathrm{E}-04$ \\
\hline f08 & separable & $3.63 E+02$ & $1.96 \mathrm{E}+02$ & - & $1.97 \mathrm{E}+03$ & $6.34 \mathrm{E}+02$ & $1.34 \mathrm{E}-02$ & $0.00 \mathrm{E}+00$ & $=$ & $1.34 \mathrm{E}-02$ & $0.00 \mathrm{E}+00$ \\
\hline f09 & separable & $4.30 \mathrm{E}-07$ & $1.17 \mathrm{E}-06$ & + & 4.93E-10 & 1.73E-10 & $0.00 \mathrm{E}+00$ & $0.00 \mathrm{E}+00$ & - & 2.27E-01 & $6.74 \mathrm{E}-01$ \\
\hline $\mathrm{f} 10$ & separable & $3.32 \mathrm{E}-05$ & $6.85 \mathrm{E}-05$ & $=$ & $5.45 \mathrm{E}-06$ & $1.33 \mathrm{E}-06$ & $2.26 \mathrm{E}-11$ & $6.14 \mathrm{E}-12$ & + & 2.17E-13 & $5.69 \mathrm{E}-14$ \\
\hline $\mathrm{f} 11$ & separable & $1.45 \mathrm{E}-02$ & $2.61 \mathrm{E}-02$ & + & 3.29E-04 & $1.80 \mathrm{E}-03$ & $0.00 \mathrm{E}+00$ & $0.00 \mathrm{E}+00$ & $=$ & $0.00 \mathrm{E}+00$ & $0.00 \mathrm{E}+00$ \\
\hline $\mathrm{f} 12$ & non-separable & 4.49E-02 & $1.35 \mathrm{E}-01$ & $=$ & $3.46 \mathrm{E}-03$ & $1.89 \mathrm{E}-02$ & $2.60 \mathrm{E}-21$ & $1.39 \mathrm{E}-21$ & + & 2.95E-25 & 2.33E-25 \\
\hline f13 & non-separable & $8.01 \mathrm{E}-09$ & 2.22E-08 & $=$ & $1.14 \mathrm{E}-11$ & $5.86 \mathrm{E}-12$ & 1.43E-30 & 7.03E-21 & - & 4.99E- 25 & $2.86 \mathrm{E}-25$ \\
\hline $\mathrm{f} 14$ & non-separable & $5.93 \mathrm{E}-01$ & $1.48 \mathrm{E}+00$ & + & 4.61E-01 & $1.36 \mathrm{E}+00$ & $5.97 \mathrm{E}-08$ & $3.99 \mathrm{E}-16$ & $=$ & 5.97E-08 & $4.81 \mathrm{E}-12$ \\
\hline $\mathrm{f} 15$ & non-separable & $1.35 \mathrm{E}-03$ & $3.56 \mathrm{E}-03$ & + & 3.52E-04 & 8.65E-05 & $1.27 \mathrm{E}-05$ & $2.95 \mathrm{E}-05$ & + & $-1.40 \mathrm{E}-08 *$ & 5.16E-20 \\
\hline f16 & non-separable & 2.19E-04 & $1.06 \mathrm{E}-03$ & + & $2.26 \mathrm{E}-15$ & $9.03 \mathrm{E}-17$ & $5.16 \mathrm{E}-11$ & $2.73 \mathrm{E}-10$ & $=$ & 1.64E-12 & $3.98 \mathrm{E}-12$ \\
\hline $\mathrm{f} 17$ & non-separable & $2.10 \mathrm{E}-05$ & $7.01 \mathrm{E}-05$ & + & 3.22E-06 & 1.62E-05 & $9.95 \mathrm{E}-16$ & $1.46 \mathrm{E}-15$ & + & $1.67 \mathrm{E}-16$ & $0.00 \mathrm{E}+00$ \\
\hline $\mathrm{f} 18$ & non-separable & $7.50 \mathrm{E}-03$ & $3.66 \mathrm{E}-02$ & + & 1.91E-15 & 1.27E-15 & $2.30 \mathrm{E}-14$ & $1.63 \mathrm{E}-14$ & + & 7.18E-15 & $7.86 \mathrm{E}-15$ \\
\hline f19 & non-separable & $1.74 \mathrm{E}-06$ & $6.24 \mathrm{E}-06$ & + & 2.55E-07 & 2.93E-09 & $2.54 \mathrm{E}-07$ & $3.88 \mathrm{E}-15$ & $=$ & $2.54 \mathrm{E}-07$ & $4.10 \mathrm{E}-15$ \\
\hline $\mathrm{f} 20$ & non-separable & $4.76 \mathrm{E}-02$ & $5.92 \mathrm{E}-02$ & + & 2.77E-02 & 5.11E-02 & $1.59 \mathrm{E}-02$ & $4.11 \mathrm{E}-02$ & $=$ & $5.03 \mathrm{E}-14$ & $2.04 \mathrm{E}-13$ \\
\hline $\mathrm{f} 21$ & non-separable & $5.09 \mathrm{E}+00$ & $3.43 \mathrm{E}+00$ & + & $2.75 E+00$ & $3.49 \mathrm{E}+00$ & $6.69 \mathrm{E}-01$ & $1.61 \mathrm{E}+00$ & + & 4.96E-07 & $1.39 \mathrm{E}-06$ \\
\hline $\mathrm{f} 22$ & non-separable & $3.34 \mathrm{E}+00$ & $3.43 \mathrm{E}+00$ & + & 4.78E-01 & $1.82 \mathrm{E}+00$ & 3.03E-04 & $1.28 \mathrm{E}-03$ & + & 1.32E-07 & 2.22E-07 \\
\hline $\mathrm{f} 23$ & non-separable & $3.16 \mathrm{E}+00$ & $3.69 \mathrm{E}+00$ & + & 4.04E-01 & $1.55 \mathrm{E}+00$ & $1.89 \mathrm{E}-05$ & $1.43 \mathrm{E}-04$ & + & $-9.68 E-06$ & $1.60 \mathrm{E}-02$ \\
\hline F01 & separable & $6.98 \mathrm{E}-10$ & $1.52 \mathrm{E}-09$ & $=$ & $4.21 \mathrm{E}-12$ & $2.09 \mathrm{E}-12$ & $0.00 \mathrm{E}+00$ & $0.00 \mathrm{E}+00$ & $=$ & $0.00 \mathrm{E}+00$ & $0.00 \mathrm{E}+00$ \\
\hline F02 & non-separable & $4.18 \mathrm{E}+00$ & $2.15 \mathrm{E}+00$ & + & 1.79E-02 & 2.47E-02 & $7.30 \mathrm{E}+02$ & $1.90 \mathrm{E}+02$ & + & 3.19E-12 & 7.10E-12 \\
\hline F03 & non-separable & $2.09 \mathrm{E}+06$ & $8.62 \mathrm{E}+05$ & + & $1.03 E+06$ & $4.65 E+05$ & $1.76 \mathrm{E}+07$ & $4.92 \mathrm{E}+06$ & + & $3.06 \mathrm{E}+05$ & $2.68 \mathrm{E}+05$ \\
\hline F04 & non-separable & $9.48 \mathrm{E}+02$ & $7.58 \mathrm{E}+02$ & + & 3.11E-01 & 2.92E-01 & $2.37 \mathrm{E}+03$ & $6.14 \mathrm{E}+02$ & + & 1.02E-04 & 2.70E-04 \\
\hline F05 & non-separable & $4.63 \mathrm{E}+03$ & $1.00 \mathrm{E}+03$ & + & 4.07E+03 & $6.22 \mathrm{E}+02$ & $5.01 \mathrm{E}+02$ & $2.16 \mathrm{E}+02$ & + & $1.15 E+02$ & $2.83 E+02$ \\
\hline F06 & non-separable & $6.82 \mathrm{E}+02$ & $2.07 \mathrm{E}+03$ & $=$ & $3.30 \mathrm{E}+02$ & $4.62 \mathrm{E}+02$ & $2.37 \mathrm{E}+01$ & $1.03 \mathrm{E}+01$ & + & $8.80 \mathrm{E}+00$ & $2.84 \mathrm{E}+00$ \\
\hline F07 & non-separable & $1.46 \mathrm{E}-02$ & $1.47 \mathrm{E}-02$ & + & $1.30 \mathrm{E}-02$ & 7.95E-03 & 6.57E-04 & $2.50 \mathrm{E}-03$ & + & $0.00 \mathrm{E}+00$ & $0.00 \mathrm{E}+00$ \\
\hline F08 & non-separable & $2.03 E+01$ & 9.33E-02 & - & $2.06 \mathrm{E}+01$ & $9.00 \mathrm{E}-02$ & $2.09 \mathrm{E}+01$ & $4.51 \mathrm{E}-02$ & $=$ & $2.09 \mathrm{E}+01$ & 7.29E-02 \\
\hline F09 & separable & $8.20 \mathrm{E}-07$ & $3.89 \mathrm{E}-06$ & + & 3.55E-10 & $1.77 \mathrm{E}-10$ & $0.00 \mathrm{E}+00$ & $0.00 \mathrm{E}+00$ & - & $5.71 \mathrm{E}-06$ & 2.87E-05 \\
\hline F10 & non-separa & $5.74 \mathrm{E}+01$ & $1.91 \mathrm{E}+01$ & $=$ & $5.35 \mathrm{E}+01$ & $1.60 \mathrm{E}+01$ & $8.09 \mathrm{E}+01$ & $9.61 \mathrm{E}+00$ & + & $6.95 E+01$ & $8.78 \mathrm{E}+00$ \\
\hline F11 & non-separable & $2.86 \mathrm{E}+01$ & $4.22 \mathrm{E}+00$ & + & $1.31 \mathrm{E}+01$ & $3.78 \mathrm{E}+00$ & $3.07 \mathrm{E}+01$ & $1.56 \mathrm{E}+00$ & + & $2.93 E+01$ & $1.69 \mathrm{E}+00$ \\
\hline F12 & non-separable & $1.13 \mathrm{E}+04$ & $8.23 \mathrm{E}+03$ & + & $6.06 E+03$ & $8.52 E+03$ & $2.47 \mathrm{E}+04$ & $1.24 \mathrm{E}+04$ & + & $5.41 E+03$ & $1.01 \mathrm{E}+04$ \\
\hline F13 & non-separab & $1.08 \mathrm{E}+00$ & $2.11 \mathrm{E}-01$ & $=$ & $1.10 \mathrm{E}+00$ & $1.69 \mathrm{E}-01$ & $2.58 \mathrm{E}+00$ & 2.59E-01 & - & $3.37 \mathrm{E}+00$ & 3.44E-01 \\
\hline F14 & non-separable & $1.35 \mathrm{E}+01$ & $2.75 \mathrm{E}-01$ & + & $1.31 \mathrm{E}+01$ & 3.49E-01 & $1.29 \mathrm{E}+01$ & $1.82 \mathrm{E}-01$ & $=$ & $1.28 \mathrm{E}+01$ & $1.88 \mathrm{E}-01$ \\
\hline$+/=/-$ & & & & & & & & & & & \\
\hline
\end{tabular}

"+ ", "-", and "=" symbolize the performance of the CMM-BBO algorithm being better or worse than, and similar to that of the existing BBO variant, respectively, according to the Wilcoxon rank-sum test at the $5 \%$ significance level.

* A negative value means that the achieved value is better than the best result provided in Table 1 . 
Table 4. Comparison of SR values between the existing BBO variants and the corresponding CMM-BBO algorithms

\begin{tabular}{|c|c|c|c|c|c|c|c|c|c|c|c|c|c|}
\hline Algorithm & & $\mathrm{rcBBO}$ & CMM-r & BBO & rcBBOg & CMM-rcBBC & & pBBO & CMM-pBE & & DE/BBO & CMM-DE/B & \\
\hline Functions & & $\mathrm{SR}$ & SR & & SR & SR & & SR & SR & & SR & SR & \\
\hline f01 & separable & 0 & 30 & + & 0 & 30 & + & 23 & 30 & + & 30 & 30 & $=$ \\
\hline f02 & non-separable & 0 & 0 & $=$ & 0 & 0 & $=$ & 6 & 0 & - & 30 & 30 & $=$ \\
\hline f03 & non-separable & 0 & 0 & $=$ & 0 & 0 & $=$ & 0 & 0 & $=$ & 0 & 30 & + \\
\hline f04 & non-separable & 0 & 0 & $=$ & 0 & 0 & $=$ & 0 & 0 & $=$ & 0 & 30 & + \\
\hline f05 & non-separable & 0 & 0 & $=$ & 0 & 0 & $=$ & 0 & 0 & $=$ & 0 & 15 & + \\
\hline f06 & separable & 2 & 30 & + & 30 & 30 & $=$ & 30 & 30 & $=$ & 30 & 30 & $\begin{array}{l}= \\
=\end{array}$ \\
\hline f07 & separable & 28 & 30 & + & 30 & 30 & $=$ & 30 & 20 & - & 30 & 30 & $=$ \\
\hline f08 & separable & 0 & 0 & $=$ & 0 & 0 & $=$ & 0 & 0 & $=$ & 0 & 0 & $=$ \\
\hline f09 & separable & 0 & 30 & + & 0 & 30 & + & 16 & 30 & + & 30 & 6 & - \\
\hline f10 & separable & 0 & 0 & $=$ & 0 & 0 & $=$ & 6 & 0 & - & 30 & 30 & $=$ \\
\hline f11 & separable & 0 & 29 & + & 0 & 24 & + & 18 & 29 & + & 30 & 30 & $=$ \\
\hline f12 & non-separable & 0 & 30 & + & 0 & 30 & + & 24 & 29 & + & 30 & 30 & $=$ \\
\hline f13 & non-separable & 0 & 30 & + & 0 & 30 & + & 26 & 30 & + & 30 & 30 & $=$ \\
\hline f14 & non-separable & 0 & 0 & + & 0 & 0 & $=$ & 0 & 0 & $=$ & 0 & 0 & $=$ \\
\hline f15 & non-separable & 0 & 0 & $=$ & 0 & 0 & $=$ & 0 & 0 & $=$ & 11 & 30 & + \\
\hline f16 & non-separable & 0 & 5 & + & 0 & 15 & + & 19 & 30 & + & 30 & 30 & $=$ \\
\hline f17 & non-separable & 0 & 1 & + & 0 & 1 & + & 10 & 23 & + & 30 & 30 & $=$ \\
\hline f18 & non-separable & 0 & 24 & + & 0 & 17 & + & 21 & 30 & + & 30 & 30 & $=$ \\
\hline f19 & non-separable & 0 & 0 & $=$ & 0 & 0 & $=$ & 0 & 0 & $=$ & 0 & 0 & $=$ \\
\hline $\mathrm{f} 20$ & non-separable & 0 & 21 & + & 0 & 25 & + & 10 & 23 & + & 26 & 30 & + \\
\hline $\mathrm{f} 21$ & non-separable & 0 & 19 & + & 0 & 21 & + & 5 & 18 & + & 0 & 0 & $=$ \\
\hline $\mathrm{f} 22$ & non-separable & 0 & 24 & + & 0 & 24 & + & 7 & 28 & + & 3 & 1 & - \\
\hline $\mathrm{f} 23$ & non-separable & 0 & 26 & + & 0 & 26 & + & 13 & 28 & + & 27 & 30 & + \\
\hline F01 & separable & 0 & 30 & + & 0 & 30 & + & 30 & 30 & $=$ & 30 & 30 & $=$ \\
\hline F02 & non-separable & 0 & 0 & $=$ & 0 & 0 & $=$ & 0 & 0 & $=$ & 0 & 30 & + \\
\hline F03 & non-separable & 0 & 0 & $=$ & 0 & 0 & $=$ & 0 & 0 & $=$ & 0 & 0 & $=$ \\
\hline F04 & non-separable & 0 & 0 & $=$ & 0 & 0 & $=$ & 0 & 0 & $=$ & 0 & 0 & $=$ \\
\hline F05 & non-separable & 0 & 0 & $=$ & 0 & 0 & $=$ & 0 & 0 & $=$ & 0 & 0 & $=$ \\
\hline F06 & non-separable & 0 & 0 & $=$ & 0 & 0 & $=$ & 0 & 0 & $=$ & 0 & 0 & $=$ \\
\hline F07 & non-separable & 0 & 0 & $=$ & 0 & 0 & $=$ & 0 & 0 & $=$ & 14 & 30 & + \\
\hline F08 & non-separable & 0 & 0 & $=$ & 0 & 0 & $=$ & 0 & 0 & $=$ & 0 & 0 & $=$ \\
\hline F09 & separable & 0 & 30 & + & 0 & 30 & + & 17 & 30 & + & 30 & 22 & - \\
\hline F10 & non-separable & 0 & 0 & $=$ & 0 & 0 & $=$ & 0 & 0 & $=$ & 0 & 0 & $=$ \\
\hline F11 & non-separable & 0 & 0 & $=$ & 0 & 0 & $=$ & 0 & 0 & $=$ & 0 & 0 & $=$ \\
\hline F12 & non-separable & 0 & 0 & $=$ & 0 & 0 & $=$ & 0 & 0 & $=$ & 0 & 0 & $=$ \\
\hline F13 & non-separable & 0 & 0 & $=$ & 0 & 0 & $=$ & 0 & 0 & $=$ & 0 & 0 & $=$ \\
\hline F14 & non-separable & 0 & 0 & $=$ & 0 & 0 & $=$ & 0 & 0 & $=$ & 0 & 0 & $=$ \\
\hline$+/=/-$ & & & & $20 / 0$ & & & $23 / 0$ & & & $21 / 3$ & & & $8 / 26 / 3$ \\
\hline
\end{tabular}

In addition, Table 5 summarizes the multiple-problem Wilcoxon signed-rank test for the CMM-BBO algorithms and the existing $\mathrm{BBO}$ variants on all the 37 benchmark functions. The Wilcoxon signed-rank test was conducted in the KEEL software[33]. In Table $6, R+$ is the sum of ranks for the functions in which the first algorithm outperforms the second, and $R$ - the sum of ranks for the opposite. p-vlaue is the smallest level of significance. As shown in Table 6, all the CMM-BBO algorithms attain higher $R+$ values than $R-$ values. Furthermore, the $p$ values in all cases are less than 0.05 , which means that the CMM-BBO algorithms are significantly better than the existing $\mathrm{BBO}$ variants. 
Table 5. Multiple-problem Wilcoxon test for the CMM-BBO algorithms and the existing BBO variants

\begin{tabular}{cccccc}
\hline Algorithm & R + & R- & p-value & $\alpha=0.05$ & $\alpha=0.1$ \\
\hline CMM-rcBBO vs rcBBO & 632 & 34 & $1.08 \mathrm{E}-07$ & Yes & Yes \\
CMM-rcBBOg vs rcBBOg & 634 & 32 & $1.65 \mathrm{E}-07$ & Yes & Yes \\
CMM-pBBO vs pBBO & 579 & 87 & $3.97 \mathrm{E}-05$ & Yes & Yes \\
CMM-DE/BBO vs DE/BBO & 572.5 & 93.5 & $6.90 \mathrm{E}-05$ & Yes & Yes \\
\hline
\end{tabular}

$R+$ is the sum of ranks for the functions in which the first algorithm outperforms the second, and $R-$ the sum of ranks for the opposite. p-vlaue is the smallest level of significance. Yes means significant difference and No means no significant difference at the given significance level.

Table 6. Average ranking of existing BBO variants and the CMM-BBO algorithms according to the Friedman tests

\begin{tabular}{ccc}
\hline Algorithm & Average ranking & Final rank \\
\hline rcBBO & 7.0946 & 8 \\
CMM-rcBBO & 4.0135 & 5 \\
rcBBOg & 6.7297 & 7 \\
CMM-rcBBOg & 3.9324 & 4 \\
pBBO & 4.8243 & 6 \\
CMM-pBBO & 3.3784 & 2 \\
DE/BBO & 3.4865 & 3 \\
CMM-DE/BBO & 2.5405 & 1 \\
\hline
\end{tabular}

To establish the rankings across all the eight $\mathrm{BBO}$ algorithms, i.e., the four existing $\mathrm{BBO}$ variants and their corresponding four CMM-BBO algorithms, on the 37 benchmark functions, the Friedman test is carried out, in which Bonferroni-Dunn's procedure was used as a post hoc procedure. As shown in Table 6, CMM-DE/BBO ranks first, followed in order by CMM-pBBO, $\mathrm{DE} / \mathrm{BBO}$, and $\mathrm{CMM}-\mathrm{rcBBOg}$.

Fig. 3 plots the convergence graphs of the eight $\mathrm{BBO}$ algorithms on 8 selected functions, namely f01, f03, f10, f12, F02, F07, F11, and F14. The convergence graphs depict the mean error curves of all the $\mathrm{BBO}$ algorithms in our numeric simulations over 30 independent runs. Overall, our developed CMM-BBO algorithms converge faster than the existing BBO variants on most of the benchmark functions.

\subsection{Comparison of CMM-DE/BBO with other EAs}

As shown above, across the eight $\mathrm{BBO}$ algorithms, $\mathrm{CMM}-\mathrm{DE} / \mathrm{BBO}$ performs the best. In this sub-section, we will further evaluate CMM-BBO by comparing CMM-DE/BBO with six other state-of-the-art EAs, namely CMAES[2], jDE[3], SaDE[4], JADE[5], CLPSO[6], and DMSPSO[7]. The Matlab source codes of CMAES, jDE, SaDE, JADE, and CLPSO were downloaded from Q. Zhang's website"http://dces.essex.ac.uk/staff/qzhang." The Matlab source code of DMSPSO was provided by P. N. Suganthan.

CMAES, proposed by Hansen and Ostermeier [2], is an evolution strategy (ES) based on completely derandomized self-adaptation. $\mathrm{jDE}, \mathrm{SaDE}$, and JADE are three representative DE algorithms. jDE, proposed by Brest [3], is a $\mathrm{DE}$ with self-adaptive control parameter. SaDE, proposed by Qin et al. [4], gradually self-adapts both mutation strategies and their associated control parameters through learning from the previous experiences in generating promising solutions. JADE, proposed by Zhang and Sanderson [5], employs a new mutation strategy "DE/current-to-pbest" with optional external archive and updates control parameters in an adaptive manner.

CLPSO and DMSPSO are two representative PSO algorithms. CLPSO, proposed by Liang et al. [6], uses all other particles' historical best information to update a particle's velocity. DMSPSO, proposed by Liang and Suganthan [7], uses dynamic multi-swarm topology to balance the 
exploration and exploitation.

The parameter settings of the six EAs are the same as in the literature, respectively, as presented in Table 7.

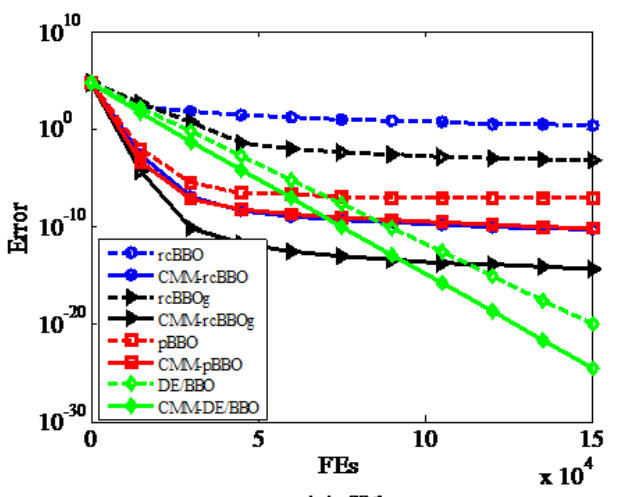

(a) f01

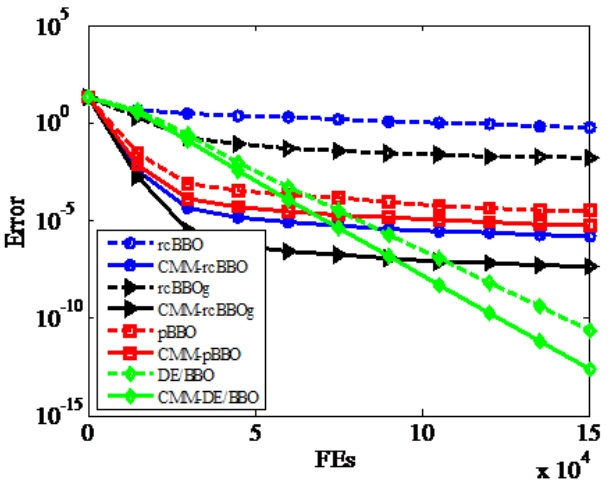

(c) f10

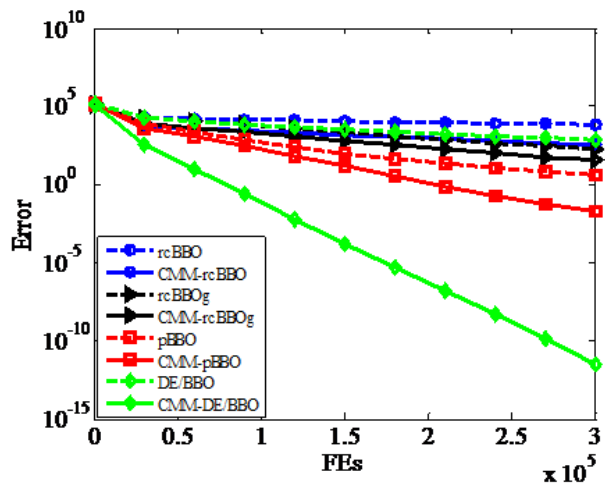

(e) F02

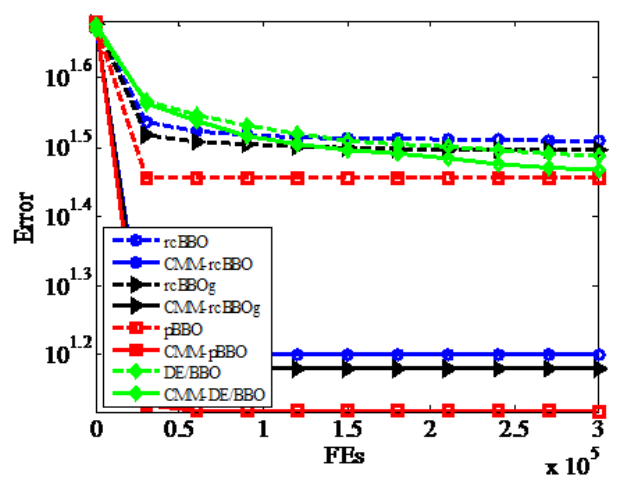

(g) F11

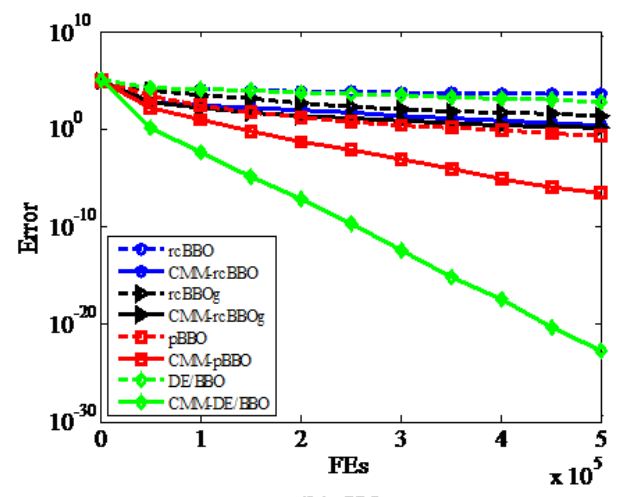

(b) $\mathrm{f} 03$

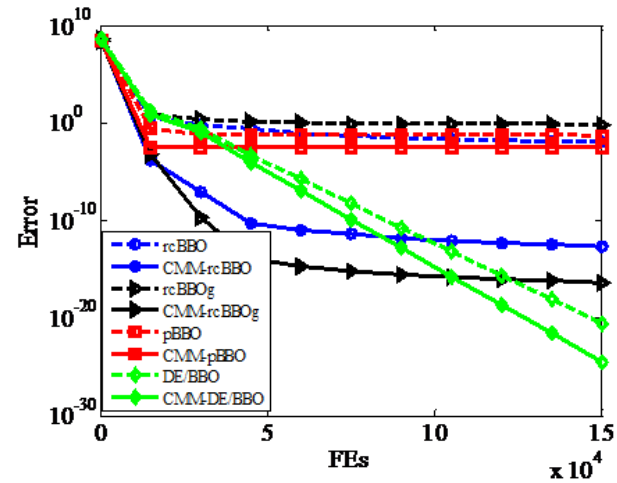

(d) $\mathrm{f12}$

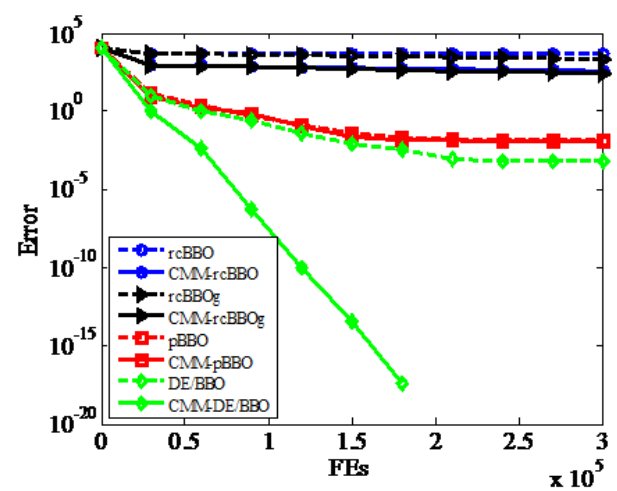

(f) $\mathrm{F07}$

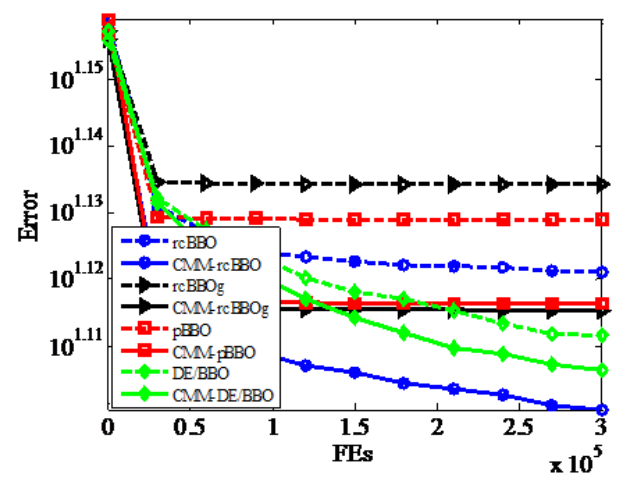

(h) F14

Fig. 3. Convergence graphs (mean curves) of all the eight $\mathrm{BBO}$ algorithms on f01, f03, f10, f12, F02, F07, F11, and F14 
Table 7. Parameter settings of the six EAs

\begin{tabular}{ll}
\hline Algorithm & Parameters \\
\hline CMAES & number of offsprings $\lambda=4+$ floor $(3 \log (D))$, number of parents $\mu=$ floor $(\lambda / 2) ;$ \\
jDE & population size $N P=100, \tau_{1}=\tau_{2}=0.1, F_{l}=0.1, F_{u}=0.9 ;$ \\
SaDE & $N P=50$, learning period $L P=50 ;$ \\
JADE & $N P=100, c=0.1, p=0.05 ;$ \\
CLPSO & population size $p s=40$, inertia weight $w$ linearly decreasing from 0.9 to 0.2 , acceleration coefficients $c=1.494$, \\
& refreshing gap $m=5 ;$ \\
DMSPSO & $p s=40, w=0.729, c_{1}=c_{2}=1.496$, population size of sub-swarm $m=5$, regrouping period $R=5$. \\
\hline
\end{tabular}

All the algorithms are evaluated on the 37 benchmark functions over 30 independent runs. Table 8 compares the errors of all the algorithms. The multiple-problem Wilcoxon signed-rank test is presented in Table 9. In addition, the rankings of the EAs according to the Friedman test are presented in Table 10. As shown in Table 10, performances of CMM-DE/BBO are significantly better than CMAES, jDE, SaDE, JADE, CLPSO, and DMSPSO on 24, 14, 14, 9, 28, and 21 functions, respectively; and similar to those of CMAES, jDE, SaDE, JADE, CLPSO, and DMSPSO on 3, 9, 7, 7, 4 and 6 functions, respectively. However, the performances of CMM-DE/BBO are significantly worse than those of CMAES, jDE, SaDE, JADE, CLPSO, and DMSPSO on 10,14, 16, 21, 5, and 10 functions, respectively. According to the multiple-problem Wilcoxon signed-rank test, CMM-DE/BBO attains higher positive-ranks $\left(R^{+}\right)$than CMAES, CLPSO and DMSPSO, and there are significant differences among these algorithms when $\alpha=0.05$ and $\alpha=0.1$. There are no significant differences among CMM-DE/BBO, jDE, SaDE, and JADE when $\alpha=0.05$ and $\alpha=0.1$. As shown in Table 10, JADE ranks the first, and CMM-DE/BBO the second, followed by jDE, SaDE, DMSPSO, CLPSO, and CMAES. Therefore, it is fair to say that $\mathrm{CMM}-\mathrm{DE} / \mathrm{BBO}$ is an effective $\mathrm{BBO}$ variant, thanks to the $\mathrm{CMM}$ operator.

\section{Discussions, Conclusions and Future Work}

$\mathrm{BBO}$ is a new bio-inspired EA which has proven its quality and versatility on a wide range of optimization problems. However, the single-feature-migration of BBO leaves it with heavy dependence upon the coordinate system, and poor performance when applied to non-separable problems.

To address this drawback of $\mathrm{BBO}$, in this paper we have proposed the covariance matrix based migration (CMM) to relieve BBO's dependence upon the coordinate system so that BBO's rotational invariance is enhanced. By use of our proposed CMM operator, the original coordinate system is rotated into an eigenvector-based one, in which habitants can share their information more efficiently.

By embedding the CMM into BBO, we have put forward a new BBO approach, namely biogeography-based optimization with covariance matrix based migration, called CMM-BBO. Specifically, four CMM-BBO algorithms, namely, CMM-rcBBO, CMM-rcBBOg, CMM-pBBO, and $\mathrm{CMM}-\mathrm{DE} / \mathrm{BBO}$, have been developed by the $\mathrm{CMM}$ operator being randomly combined with the original migration in the four selected existing $\mathrm{BBO}$ variants.

While our proposed CMM operator looks like the eigenvector-based crossover operator in DE [29], [30], there are important differences between the two. Firstly, the eigenvector-based crossover operator is designed for DE, while our CMM operator is for BBO. Secondly, the eigenvector-based crossover operator only utilizes the information of two individuals, i.e., one rotated parent individual and its corresponding child individual; but in our CMM operator, the generated individual can obtain the information from all the rotated habitants based on BBO migration. Thirdly, the core operator in $\mathrm{DE}$ is the mutation, not the crossover; while the core operator in BBO is the migration. Therefore, the CMM operator would impact upon BBO substantially more profoundly than the eigenvector-based crossover operator would upon DE. 
Table 8. Comparison of CMM-DE/BBO with other evolutionary algorithms

\begin{tabular}{|c|c|c|c|c|c|c|c|c|c|c|c|}
\hline \multirow[b]{2}{*}{ Functions } & \multicolumn{2}{|c|}{ CMM-DE/BBO } & \multicolumn{3}{|c|}{ CMAES } & \multicolumn{3}{|c|}{$\mathrm{jDE}$} & \multicolumn{3}{|c|}{$\mathrm{SaDE}$} \\
\hline & mean & SD & mean & SD & & mean & SD & & mean & SD & \\
\hline $\mathrm{f} 01$ & $2.90 \mathrm{E}-25$ & $1.59 \mathrm{E}-25$ & $5.80 \mathrm{E}-29$ & $1.33 \mathrm{E}-29$ & - & $1.10 \mathrm{E}-28$ & $8.81 \mathrm{E}-29$ & - & $1.42 \mathrm{E}-64$ & $3.36 \mathrm{E}-64$ & - \\
\hline $\mathrm{f} 02$ & $7.82 \mathrm{E}-17$ & $2.54 \mathrm{E}-17$ & $9.35 \mathrm{E}-02$ & 4.04E-01 & + & $1.38 \mathrm{E}-23$ & $8.79 \mathrm{E}-24$ & - & 7.22E-53 & $7.46 \mathrm{E}-53$ & - \\
\hline f03 & $1.53 \mathrm{E}-23$ & $4.13 \mathrm{E}-23$ & $1.57 \mathrm{E}-26$ & $2.45 \mathrm{E}-27$ & - & $2.87 \mathrm{E}-14$ & $4.44 \mathrm{E}-14$ & + & $6.34 \mathrm{E}-14$ & $7.89 \mathrm{E}-14$ & + \\
\hline f04 & $2.14 \mathrm{E}-15$ & $1.33 \mathrm{E}-15$ & 4.13E-15 & $6.01 \mathrm{E}-16$ & + & $2.08 \mathrm{E}-01$ & $3.96 \mathrm{E}-01$ & + & $5.48 \mathrm{E}-21$ & $2.89 \mathrm{E}-20$ & - \\
\hline f05 & $2.14 \mathrm{E}-01$ & $5.12 \mathrm{E}-01$ & $1.33 \mathrm{E}-01$ & $7.28 \mathrm{E}-01$ & - & $1.43 \mathrm{E}-01$ & $7.28 \mathrm{E}-01$ & - & $2.46 \mathrm{E}+01$ & $2.14 \mathrm{E}+01$ & + \\
\hline f06 & $0.00 \mathrm{E}+00$ & $0.00 \mathrm{E}+00$ & $0.00 \mathrm{E}+00$ & $0.00 \mathrm{E}+00$ & $=$ & $0.00 \mathrm{E}+00$ & $0.00 \mathrm{E}+00$ & $=$ & $0.00 \mathrm{E}+00$ & $0.00 \mathrm{E}+00$ & $=$ \\
\hline f07 & $2.66 \mathrm{E}-03$ & $7.46 \mathrm{E}-04$ & $1.97 \mathrm{E}-01$ & 7.32E-02 & + & $3.64 \mathrm{E}-03$ & $6.94 \mathrm{E}-04$ & + & $2.33 \mathrm{E}-03$ & $9.15 \mathrm{E}-04$ & $=$ \\
\hline f08 & $1.34 \mathrm{E}-02$ & $0.00 \mathrm{E}+00$ & $5.64 \mathrm{E}+03$ & $5.31 \mathrm{E}+02$ & + & $1.34 \mathrm{E}-02$ & $0.00 \mathrm{E}+00$ & $=$ & $1.34 \mathrm{E}-02$ & $0.00 \mathrm{E}+00$ & $=$ \\
\hline f09 & $2.27 \mathrm{E}-01$ & $6.74 \mathrm{E}-01$ & $2.19 \mathrm{E}+02$ & $6.27 \mathrm{E}+01$ & + & $0.00 \mathrm{E}+00$ & $0.00 \mathrm{E}+00$ & - & $6.63 \mathrm{E}-02$ & $2.52 \mathrm{E}-01$ & - \\
\hline $\mathrm{f} 10$ & $2.17 \mathrm{E}-13$ & $5.69 \mathrm{E}-14$ & $1.95 \mathrm{E}+01$ & $1.73 \mathrm{E}-01$ & + & $7.22 \mathrm{E}-15$ & $6.49 \mathrm{E}-16$ & - & $6.21 \mathrm{E}-02$ & $2.36 \mathrm{E}-01$ & + \\
\hline f11 & $0.00 \mathrm{E}+00$ & $0.00 \mathrm{E}+00$ & $1.31 \mathrm{E}-03$ & $3.02 \mathrm{E}-03$ & + & $0.00 \mathrm{E}+00$ & $0.00 \mathrm{E}+00$ & $=$ & $4.11 \mathrm{E}-03$ & $7.18 \mathrm{E}-03$ & + \\
\hline $\mathrm{f} 12$ & $2.95 \mathrm{E}-25$ & $2.33 \mathrm{E}-25$ & $6.91 \mathrm{E}-03$ & $2.63 \mathrm{E}-02$ & + & $1.00 \mathrm{E}-29$ & $1.36 \mathrm{E}-29$ & - & $6.91 \mathrm{E}-03$ & $2.63 \mathrm{E}-02$ & + \\
\hline $\mathrm{f} 13$ & $4.99 \mathrm{E}-25$ & $2.86 \mathrm{E}-25$ & $3.66 \mathrm{E}-04$ & $2.01 \mathrm{E}-03$ & + & $6.47 \mathrm{E}-29$ & $8.41 \mathrm{E}-29$ & - & $1.35 \mathrm{E}-32$ & $0.00 \mathrm{E}+00$ & - \\
\hline f14 & $5.97 \mathrm{E}-08$ & $4.81 \mathrm{E}-12$ & $1.20 \mathrm{E}+01$ & $6.97 \mathrm{E}+00$ & + & $5.97 \mathrm{E}-08$ & $2.72 \mathrm{E}-16$ & - & $1.11 \mathrm{E}-02$ & $6.06 \mathrm{E}-02$ & + \\
\hline f15 & $-1.40 \mathrm{E}-08$ & $5.16 \mathrm{E}-20$ & $4.22 \mathrm{E}-03$ & $8.06 \mathrm{E}-03$ & + & $-1.40 \mathrm{E}-08$ & $6.42 \mathrm{E}-20$ & + & $-1.40 \mathrm{E}-08$ & 7.27E-13 & $=$ \\
\hline f16 & $1.64 \mathrm{E}-12$ & $3.98 \mathrm{E}-12$ & 3.99E-01 & $8.21 \mathrm{E}-01$ & + & 7.49E-13 & $1.65 \mathrm{E}-12$ & $=$ & $2.24 \mathrm{E}-15$ & $6.78 \mathrm{E}-17$ & - \\
\hline $\mathrm{f} 17$ & $1.67 \mathrm{E}-16$ & $0.00 \mathrm{E}+00$ & $1.67 \mathrm{E}-16$ & $0.00 \mathrm{E}+00$ & $=$ & $1.67 \mathrm{E}-16$ & $0.00 \mathrm{E}+00$ & $=$ & $1.67 \mathrm{E}-16$ & $0.00 \mathrm{E}+00$ & $=$ \\
\hline f18 & $7.18 \mathrm{E}-15$ & $7.86 \mathrm{E}-15$ & $1.89 \mathrm{E}+01$ & $3.48 \mathrm{E}+01$ & + & $4.88 \mathrm{E}-15$ & $2.75 \mathrm{E}-15$ & $=$ & $2.09 \mathrm{E}-15$ & $1.19 \mathrm{E}-15$ & - \\
\hline f19 & $2.54 \mathrm{E}-07$ & $4.10 \mathrm{E}-15$ & $1.91 \mathrm{E}-01$ & $7.26 \mathrm{E}-01$ & + & $2.54 \mathrm{E}-07$ & $1.42 \mathrm{E}-15$ & - & $2.54 \mathrm{E}-07$ & $2.13 \mathrm{E}-16$ & - \\
\hline $\mathrm{f} 20$ & $5.03 \mathrm{E}-14$ & $2.04 \mathrm{E}-13$ & $2.77 \mathrm{E}-02$ & $5.11 \mathrm{E}-02$ & + & $1.59 \mathrm{E}-02$ & $4.11 \mathrm{E}-02$ & + & 7.93E-03 & $3.02 \mathrm{E}-02$ & + \\
\hline $\mathrm{f} 21$ & $4.96 \mathrm{E}-07$ & $1.39 \mathrm{E}-06$ & $4.76 \mathrm{E}+00$ & $3.30 \mathrm{E}+00$ & + & $3.44 \mathrm{E}-04$ & $1.18 \mathrm{E}-03$ & + & $-5.67 \mathrm{E}-11$ & $4.51 \mathrm{E}-12$ & - \\
\hline $\mathrm{f} 22$ & $1.32 \mathrm{E}-07$ & $2.22 \mathrm{E}-07$ & $4.65 \mathrm{E}+00$ & $3.44 \mathrm{E}+00$ & + & $1.26 \mathrm{E}-02$ & $6.73 \mathrm{E}-02$ & + & $-3.12 \mathrm{E}-11$ & $3.11 \mathrm{E}-12$ & - \\
\hline $\mathrm{f} 23$ & $-9.68 \mathrm{E}-06$ & $1.60 \mathrm{E}-02$ & $5.52 \mathrm{E}+00$ & $3.52 \mathrm{E}+00$ & + & $6.38 \mathrm{E}-06$ & $5.90 \mathrm{E}-05$ & + & $-9.82 \mathrm{E}-06$ & $4.69 \mathrm{E}-10$ & - \\
\hline F01 & $0.00 \mathrm{E}+00$ & $0.00 \mathrm{E}+00$ & $1.84 \mathrm{E}-25$ & 4.39E-26 & + & $0.00 \mathrm{E}+00$ & $0.00 \mathrm{E}+00$ & $=$ & $0.00 \mathrm{E}+00$ & $0.00 \mathrm{E}+00$ & $=$ \\
\hline F02 & $3.19 \mathrm{E}-12$ & $7.10 \mathrm{E}-12$ & $6.50 \mathrm{E}-25$ & $1.86 \mathrm{E}-25$ & - & $1.29 \mathrm{E}-06$ & $1.75 \mathrm{E}-06$ & + & $4.47 \mathrm{E}-06$ & $8.77 \mathrm{E}-06$ & + \\
\hline F03 & $3.06 \mathrm{E}+05$ & $2.68 \mathrm{E}+05$ & $5.09 \mathrm{E}-21$ & $1.26 \mathrm{E}-21$ & - & $1.71 \mathrm{E}+05$ & $1.10 \mathrm{E}+05$ & - & $4.36 \mathrm{E}+05$ & $1.73 \mathrm{E}+05$ & + \\
\hline F04 & $1.02 \mathrm{E}-04$ & $2.70 \mathrm{E}-04$ & $3.13 \mathrm{E}+05$ & $9.11 \mathrm{E}+05$ & + & $1.34 \mathrm{E}-02$ & $1.21 \mathrm{E}-02$ & + & $1.02 \mathrm{E}+02$ & $1.47 \mathrm{E}+02$ & + \\
\hline F05 & $1.15 \mathrm{E}+02$ & $2.83 \mathrm{E}+02$ & $3.13 \mathrm{E}-10$ & 8.99E-11 & - & $3.00 \mathrm{E}+02$ & $3.41 \mathrm{E}+02$ & + & $3.18 \mathrm{E}+03$ & $7.95 \mathrm{E}+02$ & - \\
\hline F06 & $8.80 \mathrm{E}+00$ & $2.84 \mathrm{E}+00$ & $6.64 \mathrm{E}-01$ & $1.51 \mathrm{E}+00$ & - & $2.50 \mathrm{E}+01$ & $2.72 \mathrm{E}+01$ & + & $4.78 \mathrm{E}+01$ & $3.91 \mathrm{E}+01$ & + \\
\hline F07 & $0.00 \mathrm{E}+00$ & $0.00 \mathrm{E}+00$ & $3.61 \mathrm{E}-03$ & $6.36 \mathrm{E}-03$ & + & $4.70 \mathrm{E}+03$ & $5.34 \mathrm{E}-13$ & + & $1.51 \mathrm{E}-02$ & $1.06 \mathrm{E}-02$ & - \\
\hline F08 & $2.09 \mathrm{E}+01$ & 7.29E-02 & $2.03 \mathrm{E}+01$ & $5.60 \mathrm{E}-01$ & - & $2.09 \mathrm{E}+01$ & $5.73 \mathrm{E}-02$ & $=$ & $2.10 \mathrm{E}+01$ & $4.10 \mathrm{E}-02$ & + \\
\hline F09 & $5.71 \mathrm{E}-06$ & $2.87 \mathrm{E}-05$ & $3.74 \mathrm{E}+02$ & $1.22 \mathrm{E}+02$ & + & $0.00 \mathrm{E}+00$ & $0.00 \mathrm{E}+00$ & - & $6.63 \mathrm{E}-02$ & $2.52 \mathrm{E}-01$ & + \\
\hline F10 & $6.95 \mathrm{E}+01$ & $8.78 \mathrm{E}+00$ & $4.61 \mathrm{E}+01$ & $1.17 \mathrm{E}+01$ & - & $5.57 \mathrm{E}+01$ & $8.83 \mathrm{E}+00$ & - & $4.79 \mathrm{E}+01$ & $9.64 \mathrm{E}+00$ & - \\
\hline F11 & $2.93 \mathrm{E}+01$ & $1.69 \mathrm{E}+00$ & $6.26 \mathrm{E}+00$ & $2.66 \mathrm{E}+00$ & - & $2.79 \mathrm{E}+01$ & $2.24 \mathrm{E}+00$ & - & $1.73 \mathrm{E}+01$ & $2.76 \mathrm{E}+00$ & - \\
\hline F12 & $5.41 \mathrm{E}+03$ & $1.01 \mathrm{E}+04$ & $1.16 \mathrm{E}+04$ & $1.25 \mathrm{E}+04$ & + & $5.13 \mathrm{E}+03$ & $6.19 \mathrm{E}+03$ & $=$ & $3.23 \mathrm{E}+03$ & $3.15 \mathrm{E}+03$ & $=$ \\
\hline F13 & $3.37 \mathrm{E}+00$ & $3.44 \mathrm{E}-01$ & $3.42 \mathrm{E}+00$ & 8.64E-01 & $=$ & $1.72 \mathrm{E}+00$ & $1.51 \mathrm{E}-01$ & - & $4.03 \mathrm{E}+00$ & $3.71 \mathrm{E}-01$ & + \\
\hline F14 & $1.28 \mathrm{E}+01$ & $1.88 \mathrm{E}-01$ & $1.47 \mathrm{E}+01$ & $2.95 \mathrm{E}-01$ & + & $1.30 \mathrm{E}+01$ & $1.71 \mathrm{E}-01$ & + & $1.27 \mathrm{E}+01$ & $2.35 \mathrm{E}-01$ & - \\
\hline$+=/-$ & & & & & & & $14 / 9$ & & & & \\
\hline
\end{tabular}


Table 8. (Continued)

\begin{tabular}{|c|c|c|c|c|c|c|c|c|}
\hline \multicolumn{3}{|c|}{ JADE } & \multicolumn{3}{|c|}{ CLPSO } & \multicolumn{3}{|c|}{ DMSPSO } \\
\hline mean & SD & & mean & SD & & mean & SD & \\
\hline $1.05 \mathrm{E}-56$ & $5.51 \mathrm{E}-56$ & - & $6.70 \mathrm{E}-11$ & $2.93 \mathrm{E}-11$ & + & $7.87 \mathrm{E}-47$ & $1.05 \mathrm{E}-46$ & - \\
\hline $5.10 \mathrm{E}-25$ & $2.75 \mathrm{E}-24$ & - & $3.75 \mathrm{E}-10$ & $1.15 \mathrm{E}-10$ & + & $3.80 \mathrm{E}-36$ & $8.78 \mathrm{E}-36$ & - \\
\hline $1.06 \mathrm{E}-85$ & $5.50 \mathrm{E}-85$ & - & $6.25 \mathrm{E}+01$ & $2.00 \mathrm{E}+01$ & + & 4.46E-09 & $5.71 \mathrm{E}-09$ & + \\
\hline $5.77 \mathrm{E}-66$ & $1.48 \mathrm{E}-65$ & - & 4.63E-01 & $7.06 \mathrm{E}-02$ & + & $2.86 \mathrm{E}-13$ & $5.21 \mathrm{E}-13$ & + \\
\hline $9.92 \mathrm{E}-31$ & $2.91 \mathrm{E}-30$ & - & $2.36 \mathrm{E}-01$ & $2.25 \mathrm{E}-01$ & + & $4.41 \mathrm{E}+00$ & $2.70 \mathrm{E}+00$ & + \\
\hline $0.00 \mathrm{E}+00$ & $0.00 \mathrm{E}+00$ & $=$ & $0.00 \mathrm{E}+00$ & $0.00 \mathrm{E}+00$ & $=$ & $0.00 \mathrm{E}+00$ & $0.00 \mathrm{E}+00$ & $=$ \\
\hline $6.34 \mathrm{E}-04$ & $2.40 \mathrm{E}-04$ & - & $3.20 \mathrm{E}-03$ & $7.26 \mathrm{E}-04$ & + & $2.79 \mathrm{E}-03$ & 7.36E-04 & $=$ \\
\hline $1.34 \mathrm{E}-02$ & $0.00 \mathrm{E}+00$ & $=$ & $1.34 \mathrm{E}-02$ & $4.61 \mathrm{E}-13$ & $=$ & $3.92 \mathrm{E}+03$ & $7.26 \mathrm{E}+02$ & + \\
\hline $0.00 \mathrm{E}+00$ & $0.00 \mathrm{E}+00$ & - & $0.00 \mathrm{E}+00$ & $0.00 \mathrm{E}+00$ & - & $2.43 \mathrm{E}+01$ & $6.61 \mathrm{E}+00$ & + \\
\hline $3.67 \mathrm{E}-15$ & $6.49 \mathrm{E}-16$ & - & $5.32 \mathrm{E}-06$ & $1.36 \mathrm{E}-06$ & + & $6.28 \mathrm{E}-15$ & $1.53 \mathrm{E}-15$ & - \\
\hline $0.00 \mathrm{E}+00$ & $0.00 \mathrm{E}+00$ & $=$ & $4.53 \mathrm{E}-11$ & $5.47 \mathrm{E}-11$ & + & $5.50 \mathrm{E}-03$ & $8.32 \mathrm{E}-03$ & + \\
\hline $1.57 \mathrm{E}-32$ & $5.57 \mathrm{E}-48$ & - & $2.87 \mathrm{E}-12$ & $1.44 \mathrm{E}-12$ & + & $3.46 \mathrm{E}-03$ & $1.89 \mathrm{E}-02$ & + \\
\hline $1.35 \mathrm{E}-32$ & $5.57 \mathrm{E}-48$ & - & $7.31 \mathrm{E}-11$ & $3.35 \mathrm{E}-11$ & + & $2.20 \mathrm{E}-03$ & 4.47E-03 & + \\
\hline $5.97 \mathrm{E}-08$ & $1.11 \mathrm{E}-14$ & $=$ & $5.97 \mathrm{E}-08$ & $2.22 \mathrm{E}-11$ & + & $1.33 \mathrm{E}-01$ & $1.65 \mathrm{E}-01$ & $=$ \\
\hline$-1.40 \mathrm{E}-08$ & $1.32 \mathrm{E}-19$ & $=$ & $2.07 \mathrm{E}-04$ & $8.23 \mathrm{E}-05$ & + & $-1.40 \mathrm{E}-08$ & $1.77 \mathrm{E}-19$ & + \\
\hline $1.29 \mathrm{E}-09$ & 3.83E-09 & + & $4.86 \mathrm{E}-10$ & $1.11 \mathrm{E}-09$ & + & $4.78 \mathrm{E}-12$ & $2.23 \mathrm{E}-11$ & + \\
\hline 7.59E-16 & $1.26 \mathrm{E}-15$ & + & $9.45 \mathrm{E}-12$ & $3.73 \mathrm{E}-11$ & + & $1.67 \mathrm{E}-16$ & $0.00 \mathrm{E}+00$ & $=$ \\
\hline $3.75 \mathrm{E}-14$ & $1.93 \mathrm{E}-14$ & + & $2.14 \mathrm{E}-11$ & $7.38 \mathrm{E}-11$ & + & $3.89 \mathrm{E}-15$ & $1.62 \mathrm{E}-15$ & $=$ \\
\hline $2.54 \mathrm{E}-07$ & $5.02 \mathrm{E}-14$ & + & $2.54 \mathrm{E}-07$ & 4.67E-11 & + & $2.54 \mathrm{E}-07$ & $0.00 \mathrm{E}+00$ & - \\
\hline $1.98 \mathrm{E}-02$ & $4.51 \mathrm{E}-02$ & + & $2.54 \mathrm{E}-06$ & $4.95 \mathrm{E}-06$ & + & $3.96 \mathrm{E}-03$ & $2.17 \mathrm{E}-02$ & + \\
\hline $1.72 \mathrm{E}-01$ & $9.22 \mathrm{E}-01$ & + & $2.38 \mathrm{E}-01$ & $4.70 \mathrm{E}-01$ & + & $5.00 \mathrm{E}-01$ & $1.90 \mathrm{E}+00$ & + \\
\hline $7.69 \mathrm{E}-04$ & $3.60 \mathrm{E}-03$ & + & $3.46 \mathrm{E}-01$ & 7.73E-01 & + & $-3.07 \mathrm{E}-11$ & $5.41 \mathrm{E}-12$ & - \\
\hline $3.32 \mathrm{E}-04$ & $1.46 \mathrm{E}-03$ & + & 3.69E-01 & $9.99 \mathrm{E}-01$ & + & $-9.82 \mathrm{E}-06$ & 4.30E-14 & - \\
\hline $0.00 \mathrm{E}+00$ & $0.00 \mathrm{E}+00$ & $=$ & $6.02 \mathrm{E}-26$ & $3.94 \mathrm{E}-26$ & + & $1.68 \mathrm{E}-30$ & $9.22 \mathrm{E}-30$ & $=$ \\
\hline $1.06 \mathrm{E}-28$ & $9.88 \mathrm{E}-29$ & - & $1.02 \mathrm{E}+03$ & $2.31 \mathrm{E}+02$ & + & $5.10 \mathrm{E}-04$ & $6.98 \mathrm{E}-04$ & + \\
\hline $8.26 \mathrm{E}+03$ & $7.17 \mathrm{E}+03$ & - & $1.64 \mathrm{E}+07$ & $4.58 \mathrm{E}+06$ & + & $1.09 \mathrm{E}+06$ & $4.51 \mathrm{E}+05$ & + \\
\hline $9.42 \mathrm{E}-15$ & $4.55 \mathrm{E}-14$ & - & $6.63 \mathrm{E}+03$ & $1.69 \mathrm{E}+03$ & + & $7.18 \mathrm{E}+01$ & $5.89 \mathrm{E}+01$ & + \\
\hline 6.71E-08 & $2.59 \mathrm{E}-07$ & - & $4.15 \mathrm{E}+03$ & $3.93 \mathrm{E}+02$ & + & $2.84 \mathrm{E}+03$ & $5.01 \mathrm{E}+02$ & + \\
\hline $5.36 \mathrm{E}+00$ & $1.98 \mathrm{E}+01$ & - & $2.16 \mathrm{E}+00$ & $5.08 \mathrm{E}+00$ & - & $2.04 \mathrm{E}+01$ & $2.46 \mathrm{E}+01$ & + \\
\hline $6.08 \mathrm{E}-03$ & $5.28 \mathrm{E}-03$ & + & $9.83 \mathrm{E}-01$ & $6.04 \mathrm{E}-02$ & + & $4.70 \mathrm{E}+03$ & $1.53 \mathrm{E}-12$ & + \\
\hline $2.09 \mathrm{E}+01$ & $1.84 \mathrm{E}-01$ & $=$ & $2.09 \mathrm{E}+01$ & $5.88 \mathrm{E}-02$ & $=$ & $2.08 \mathrm{E}+01$ & $5.82 \mathrm{E}-02$ & - \\
\hline $0.00 \mathrm{E}+00$ & $0.00 \mathrm{E}+00$ & - & $0.00 \mathrm{E}+00$ & $0.00 \mathrm{E}+00$ & - & $4.22 \mathrm{E}+01$ & $9.79 \mathrm{E}+00$ & + \\
\hline $2.32 \mathrm{E}+01$ & $5.04 \mathrm{E}+00$ & - & $1.14 \mathrm{E}+02$ & $1.34 \mathrm{E}+01$ & + & $5.98 \mathrm{E}+01$ & $1.17 \mathrm{E}+01$ & - \\
\hline $2.54 \mathrm{E}+01$ & $1.76 \mathrm{E}+00$ & - & $2.60 \mathrm{E}+01$ & $1.96 \mathrm{E}+00$ & - & $1.39 \mathrm{E}+01$ & $3.00 \mathrm{E}+00$ & - \\
\hline $5.41 \mathrm{E}+03$ & $4.40 \mathrm{E}+03$ & - & $1.51 \mathrm{E}+04$ & $5.51 \mathrm{E}+03$ & + & $6.90 \mathrm{E}+03$ & $7.20 \mathrm{E}+03$ & + \\
\hline $1.50 \mathrm{E}+00$ & $9.68 \mathrm{E}-02$ & - & $1.93 \mathrm{E}+00$ & $1.83 \mathrm{E}-01$ & - & $4.74 \mathrm{E}+00$ & $1.46 \mathrm{E}+00$ & + \\
\hline $1.23 \mathrm{E}+01$ & $3.15 \mathrm{E}-01$ & - & $1.28 \mathrm{E}+01$ & $1.87 \mathrm{E}-01$ & $=$ & $1.18 \mathrm{E}+01$ & $3.85 \mathrm{E}-01$ & - \\
\hline & & & & & & & & \\
\hline
\end{tabular}

"+ ", "-", and "=" symbolize the performance of the CMM-DE/BBO algorithm being better or worse than, and similar to that of the EA, respectively, according to the Wilcoxon rank-sum test at the $5 \%$ significance level. 
Table 9. Multiple-problem Wilcoxon signed-rank test for CMAES, jDE, SaDE, JADE, CLPSO, DMSPSO, and CMM-DE/BBO

\begin{tabular}{llllll}
\hline Algorithm & $\mathrm{R}+$ & $\mathrm{R}-$ & $\mathrm{p}$-value & $\alpha=0.05$ & $\alpha=0.1$ \\
\hline CMM-DE/BBO vs CMAES & 499.5 & 203.5 & $2.48 \mathrm{E}-2$ & Yes & Yes \\
CMM-DE/BBO vs jDE & 331 & 335 & $\geq 0.2$ & No & No \\
CMM-DE/BBO vs SaDE & 421 & 282 & $\geq 0.2$ & No & No \\
CMM-DE/BBO vs JADE & 190 & 476 & $\geq 0.2$ & No & No \\
CMM-DE/BBO vs CLPSO & 565.5 & 137.5 & $8.54 \mathrm{E}-04$ & Yes & Yes \\
CMM-DE/BBO vs DMSPSO & 540 & 163 & $3.71 \mathrm{E}-03$ & Yes & Yes
\end{tabular}

$R+$ is the sum of ranks for the functions in which the first algorithm outperforms the second, and $R-$ the sum of ranks for the opposite. $\mathrm{p}$-vlaue is the smallest level of significance. Yes means significant difference and No means no significant difference at the given significance level.

Table 10. Average ranking of CMAES, jDE, SaDE, JADE, CLPSO, DMSPSO, and CMM-DE/BBO according to the Friedman tests

\begin{tabular}{ccc}
\hline & Average ranking & Final rank \\
\hline CMM-DE/BBO & 3.3784 & 2 \\
CMAES & 4.9324 & 7 \\
jDE & 3.7432 & 3 \\
SaDE & 4.027 & 4 \\
JADE & 3.0135 & 1 \\
CLPSO & 4.4865 & 6 \\
DMSPSO & 4.4189 & 5 \\
\hline
\end{tabular}

The comprehensive numeric results ${ }^{1}$ we have carried out have shown that our proposed CMM-BBO approach significantly improves the performances of the existing $\mathrm{BBO}$ algorithms on most of the benchmark functions.

By now we can rightly draw the following conclusions.

i. The covariance matrix based migration $(\mathrm{CMM})$ significantly enhances the rotational invariance of BBO.

ii. The CMM operator can be easily applied to existing $\mathrm{BBO}$ variants. It can be embedded into any $\mathrm{BBO}$ variants without the framework of CMM-BBO having necessarily to be changed. Therefore, CMM-BBO approach serves as a unified framework for all kinds of $\mathrm{BBO}$ variants.

iii. The CMM-BBO approach effectively improves the performances of the existing BBO variants on non-separable as well as separable problems. Overall, the CMM-BBO algorithms have better performances than the existing $\mathrm{BBO}$ variants in terms of the performance criteria of error, SR, and convergence.

iv. According to the Friedman test, the CMM-DE/BBO algorithm ranks the first across all the eight $\mathrm{BBO}$ algorithms, i.e., rcBBO, rcBBOg, pBBO, DE/BBO, and CMM-rcBBO, CMM-rcBBOg, CMM-pBBO, CMM-DE/BBO.

v. Compared with six other representative EAs, including CMAES, jDE, SaDE, JADE, CLPSO and DMSPSO, CMM-DE/BBO achieves highly competitive results on the 37 benchmark functions. According to the Friedman test, CMM-DE/BBO ranks the second, only after JADE.

Several aspects may be worth exploring in the future work. Firstly, adaptive or self-adaptive adjusting mechanism may be designed for control parameter $P_{e}$ and its impact on performance may be studied. Secondly, Markov theory may be applied for analysis of CMM-BBO algorithms. Thirdly, computation of the covariance matrix is time-consuming in large-scale problems. How to

\footnotetext{
${ }^{1}$ The source codes of the CMM-BBO algorithms are available from the first author upon request.
} 
increase the computational efficiency of CMM-BBO algorithms would be of significance for large-scale optimization problems. Finally, it is also interesting to apply CMM-BBO to challenging real-world problems.

\section{Acknowledgments}

The first author would like to acknowledge the supports by the Research Talents Startup Foundation of Jiangsu University (grant number 15JDG139).

The authors would like to thank Dr. N. Hansen, Dr. J. Brest, and Dr. J. Zhang for providing the source codes of CMAES, jDE, and JADE, and Dr. P. N. Suganthan for providing the source codes of SaDE, CLPSO, and DMSPSO.

\section{Reference}

[1] T. Park, K.R. Ryu, A dual-population genetic algorithm for adaptive diversity control, Evolutionary Computation, IEEE Transactions on, 14 (2010) 865-884.

[2] N. Hansen, A. Ostermeier, Completely derandomized self-adaptation in evolution strategies, Evolutionary computation, 9 (2001) 159-195.

[3] J. Brest, S. Greiner, B. Bošković, M. Mernik, V. Zumer, Self-adapting control parameters in differential evolution: a comparative study on numerical benchmark problems, Evolutionary Computation, IEEE Transactions on, 10 (2006) 646-657.

[4] A.K. Qin, V.L. Huang, P.N. Suganthan, Differential evolution algorithm with strategy adaptation for global numerical optimization, Evolutionary Computation, IEEE Transactions on, 13 (2009) 398-417.

[5] J. Zhang, A.C. Sanderson, JADE: adaptive differential evolution with optional external archive, Evolutionary Computation, IEEE Transactions on, 13 (2009) 945-958.

[6] J.J. Liang, A.K. Qin, P.N. Suganthan, S. Baskar, Comprehensive learning particle swarm optimizer for global optimization of multimodal functions, Evolutionary Computation, IEEE Transactions on, 10 (2006) 281-295.

[7] J.J. Liang, P.N. Suganthan, Dynamic multi-swarm particle swarm optimizer, in: Swarm Intelligence Symposium, 2005. SIS 2005. Proceedings 2005 IEEE, IEEE, 2005, pp. 124-129.

[8] D. Simon, Biogeography-based optimization, Evolutionary Computation, IEEE Transactions on, 12 (2008) 702-713.

[9] Y.-J. Zheng, H.-F. Ling, H.-H. Shi, H.-S. Chen, S.-Y. Chen, Emergency railway wagon scheduling by hybrid biogeography-based optimization, Computers \& Operations Research, 43 (2014) 1-8.

[10] Y.-J. Zheng, H.-F. Ling, S.-Y. Chen, J.-Y. Xue, A hybrid neuro-fuzzy network based on differential biogeography-based optimization for online population classification in earthquakes, IEEE Transaction on Fuzzy Systems, 23 (2015) 1070-1083.

[11] M. Tamjidy, S. Paslar, B.H.T. Baharudin, T.S. Hong, M. Ariffin, Biogeography based optimization (BBO) algorithm to minimise non-productive time during hole-making process, International Journal of Production Research, 53 (2015) 1880-1894.

[12] Y.-J. Zheng, H.-F. Ling, J.-Y. Xue, Ecogeography-based optimization: enhancing biogeography-based optimization with ecogeographic barriers and differentiations, Computers \& Operations Research, 50 (2014) 115-127.

[13] W. Gong, Z. Cai, C.X. Ling, H. Li, A real-coded biogeography-based optimization with mutation, Applied Mathematics and Computation, 216 (2010) 2749-2758.

[14] X. Li, M. Yin, Multi-operator based biogeography based optimization with mutation for global numerical optimization, Computers \& Mathematics with Applications, 64 (2012) 2833-2844.

[15] G. Xiong, D. Shi, X. Duan, Enhancing the performance of biogeography-based optimization using polyphyletic migration operator and orthogonal learning, Computers \& Operations Research, 41 (2014) 125-139.

[16] X. Li, J. Wang, J. Zhou, M. Yin, A perturb biogeography based optimization with mutation for global numerical optimization, Applied Mathematics and Computation, 218 (2011) 598-609.

[17] H. Ma, D. Simon, Blended biogeography-based optimization for constrained optimization, 
Engineering Applications of Artificial Intelligence, 24 (2011) 517-525.

[18] D. Simon, M.G. Omran, M. Clerc, Linearized biogeography-based optimization with re-initialization and local search, Information Sciences, 267 (2014) 140-157.

[19] D. Du, D. Simon, M. Ergezer, Biogeography-based optimization combined with evolutionary strategy and immigration refusal, in: Systems, Man and Cybernetics, 2009. SMC 2009. IEEE International Conference on, IEEE, 2009, pp. 997-1002.

[20] W. Gong, Z. Cai, C.X. Ling, DE/BBO: a hybrid differential evolution with biogeography-based optimization for global numerical optimization, Soft Computing, 15 (2010) 645-665.

[21] I. Boussaïd, A. Chatterjee, P. Siarry, M. Ahmed-Nacer, Two-stage update biogeography-based optimization using differential evolution algorithm (DBBO), Computers \& Operations Research, 38 (2011) 1188-1198.

[22] H. Kundra, M. Sood, Cross-country path finding using hybrid approach of PSO and BBO, International Journal of Computer Applications, 7 (2010) 15-19.

[23] P. Savsani, R. Jhala, V. Savsani, Effect of hybridizing Biogeography-Based Optimization (BBO) technique with Artificial Immune Algorithm (AIA) and Ant Colony Optimization (ACO), Applied Soft Computing, 21 (2014) 542-553.

[24] Y.-J. Zheng, H.-F. Ling, X.-B. Wu, J.-Y. Xue, Localized biogeography-based optimization, Soft Computing, 18 (2014) 2323-2334.

[25] X.-w. Zheng, D.-j. Lu, X.-g. Wang, H. Liu, A cooperative coevolutionary biogeography-based optimizer, Applied Intelligence, 43 (2015) 95-111.

[26] H. Ma, M. Fei, Z. Ding, J. Jin, Biogeography-based optimization with ensemble of migration models for global numerical optimization, in: Evolutionary Computation (CEC), 2012 IEEE Congress on, IEEE, 2012, pp. 1-8.

[27] M. Ergezer, D. Simon, D. Du, Oppositional biogeography-based optimization, in: Systems, Man and Cybernetics, 2009. SMC 2009. IEEE International Conference on, IEEE, 2009, pp. 1009-1014.

[28] S. Saremi, S. Mirjalili, A. Lewis, Biogeography-based optimisation with chaos, Neural Computing and Applications, 25 (2014) 1077-1097.

[29] Y. Wang, H.-X. Li, T. Huang, L. Li, Differential evolution based on covariance matrix learning and bimodal distribution parameter setting, Applied Soft Computing, 18 (2014) 232-247.

[30] S.-M. Guo, C.-C. Yang, Enhancing differential evolution utilizing eigenvector-based crossover operator, Evolutionary Computation, IEEE Transactions on, 19 (2015) 31-49.

[31] X. Yao, Y. Liu, G. Lin, Evolutionary programming made faster, Evolutionary Computation, IEEE Transactions on, 3 (1999) 82-102.

[32] P.N. Suganthan, N. Hansen, J.J. Liang, K. Deb, Y.-P. Chen, A. Auger, S. Tiwari, Problem definitions and evaluation criteria for the CEC 2005 special session on real-parameter optimization, KanGAL report, 2005005 (2005).

[33] J. Alcala-Fdez, L. Sanchez, S. Garcia, M.J. del Jesus, S. Ventura, J. Garrell, J. Otero, C. Romero, J. Bacardit, V.M. Rivas, KEEL: a software tool to assess evolutionary algorithms for data mining problems, Soft Computing, 13 (2009) 307-318. 


\section{Appendix A: Sensitivity Analysis of Parameter $\boldsymbol{P}_{\mathrm{e}}$}

In order to analyze the sensitivity of parameter $P_{e}$, we have evaluated the four CMM-BBO algorithms with different $P_{e}: 0,0.1,0.2,0.3,0.4,0.5,0.6,0.7,0.8,0.9$, and 1.0. The sensitivity analysis of parameter $P_{e}$ is detailed in Tables A-1 $\sim$ A-4.

Table A-5 presents the rankings of the four CMM-BBO algorithms with different $P_{e}$ according to the Friedman test on all the 37 benchmark functions. As shown in Table A-5, for CMM-rcBBO, the best ranking is achieved when $P_{e}$ is 0.7 , followed by 0.5 and 0.4 . For CMM-rcBBOg, the best ranking is achieved when $P_{e}$ is 0.5 , followed by 0.4 and 0.7 . For CMM-pBBO, the best ranking occurs when $P_{e}=0.5$, followed by $P_{e}=0.7$ and $P_{e}=0.4$. For CMM-DE/BBO, the best ranking occurs when $P_{e}$ is 0.5 , followed by 0.7 and 0.4 .

Considering both the benchmark functions and the four CMM-BBO algorithms, $P_{e}=0.5$ seems to be the common appropriate value. 
Table A-1 Mean errors of CMM-rcBBO with different $P_{e}$ on the 37 benchmark functions

\begin{tabular}{|c|c|c|c|c|c|c|c|c|c|c|c|}
\hline$P_{e}$ & 0.0 & 0.1 & 0.2 & 0.3 & 0.4 & 0.5 & 0.6 & 0.7 & 0.8 & 0.9 & 1.0 \\
\hline f01 & $2.10 \mathrm{E}+00$ & $1.63 \mathrm{E}-08$ & $1.88 \mathrm{E}-19$ & 5.95E-15 & $1.68 \mathrm{E}-12$ & $4.49 \mathrm{E}-11$ & $5.29 \mathrm{E}-10$ & $1.97 \mathrm{E}-09$ & $9.69 \mathrm{E}-09$ & $2.14 \mathrm{E}-08$ & $4.86 \mathrm{E}-08$ \\
\hline f02 & $3.92 \mathrm{E}-01$ & 7.06E-08 & $5.02 \mathrm{E}-12$ & $3.59 \mathrm{E}-09$ & $1.10 \mathrm{E}-07$ & $6.90 \mathrm{E}-07$ & $3.26 \mathrm{E}-06$ & 7.67E-06 & $1.66 \mathrm{E}-05$ & $2.74 \mathrm{E}-05$ & $4.23 \mathrm{E}-05$ \\
\hline f03 & $3.74 \mathrm{E}+03$ & $6.62 \mathrm{E}+02$ & $1.07 \mathrm{E}+02$ & $3.03 \mathrm{E}+01$ & $8.79 \mathrm{E}+00$ & $2.04 \mathrm{E}+00$ & $5.01 \mathrm{E}-01$ & $1.36 \mathrm{E}-01$ & $7.42 \mathrm{E}-03$ & $2.70 \mathrm{E}-04$ & 1.15E-04 \\
\hline f04 & $1.39 \mathrm{E}+00$ & $6.07 \mathrm{E}-01$ & $3.70 \mathrm{E}-01$ & $9.96 \mathrm{E}-02$ & 3.69E-02 & $6.75 \mathrm{E}-03$ & 7.07E-03 & $9.90 \mathrm{E}-05$ & 3.44E-04 & $2.08 \mathrm{E}-04$ & $9.61 \mathrm{E}-04$ \\
\hline f05 & $1.19 \mathrm{E}+02$ & $6.31 \mathrm{E}+01$ & $6.11 \mathrm{E}+01$ & $4.73 \mathrm{E}+01$ & $4.52 \mathrm{E}+01$ & $3.73 \mathrm{E}+01$ & $3.87 \mathrm{E}+01$ & $2.73 \mathrm{E}+01$ & $2.88 \mathrm{E}+01$ & $3.28 \mathrm{E}+01$ & $2.98 \mathrm{E}+01$ \\
\hline f06 & $2.23 \mathrm{E}+00$ & $0.00 \mathrm{E}+00$ & $0.00 \mathrm{E}+00$ & $0.00 \mathrm{E}+00$ & $0.00 \mathrm{E}+00$ & $0.00 \mathrm{E}+00$ & $0.00 \mathrm{E}+00$ & $0.00 \mathrm{E}+00$ & $0.00 \mathrm{E}+00$ & $0.00 \mathrm{E}+00$ & $0.00 \mathrm{E}+00$ \\
\hline f07 & $5.49 \mathrm{E}-03$ & $9.42 \mathrm{E}-04$ & $1.26 \mathrm{E}-03$ & $1.58 \mathrm{E}-03$ & 2.08E- 03 & $2.05 \mathrm{E}-03$ & $2.19 \mathrm{E}-03$ & $2.29 \mathrm{E}-03$ & $2.54 \mathrm{E}-03$ & $2.71 \mathrm{E}-03$ & $2.77 \mathrm{E}-03$ \\
\hline $\mathrm{f} 08$ & $1.52 \mathrm{E}+00$ & $1.34 \mathrm{E}-02$ & $1.34 \mathrm{E}-02$ & $1.34 \mathrm{E}-02$ & $1.34 \mathrm{E}-02$ & $1.34 \mathrm{E}-02$ & $1.34 \mathrm{E}-02$ & $1.34 \mathrm{E}-02$ & $1.34 \mathrm{E}-02$ & $1.34 \mathrm{E}-02$ & $1.34 \mathrm{E}-02$ \\
\hline f09 & $2.41 \mathrm{E}-01$ & $6.44 \mathrm{E}-12$ & $0.00 \mathrm{E}+00$ & $0.00 \mathrm{E}+00$ & $1.15 \mathrm{E}-13$ & $8.41 \mathrm{E}-12$ & $1.32 \mathrm{E}-10$ & $1.12 \mathrm{E}-09$ & $3.83 \mathrm{E}-09$ & $1.91 \mathrm{E}-08$ & $2.52 \mathrm{E}-08$ \\
\hline f10 & $6.05 \mathrm{E}-01$ & 4.68E-05 & $8.26 \mathrm{E}-11$ & $1.52 \mathrm{E}-08$ & 2.24E-07 & $1.49 \mathrm{E}-06$ & 4.79E-06 & $1.06 \mathrm{E}-05$ & $1.92 \mathrm{E}-05$ & $3.17 \mathrm{E}-05$ & 5.43E-05 \\
\hline f11 & $8.12 \mathrm{E}-01$ & $1.16 \mathrm{E}-02$ & $9.04 \mathrm{E}-04$ & $3.29 \mathrm{E}-04$ & $6.57 \mathrm{E}-04$ & $2.47 \mathrm{E}-04$ & $2.47 \mathrm{E}-04$ & $2.70 \mathrm{E}-03$ & $1.15 \mathrm{E}-03$ & $8.76 \mathrm{E}-03$ & 7.47E-03 \\
\hline f12 & $1.06 \mathrm{E}-02$ & $5.36 \mathrm{E}-09$ & $2.51 \mathrm{E}-20$ & $2.78 \mathrm{E}-17$ & $6.62 \mathrm{E}-15$ & $2.11 \mathrm{E}-13$ & 1.61E-12 & $1.65 \mathrm{E}-11$ & $5.43 \mathrm{E}-11$ & $1.23 \mathrm{E}-10$ & 4.82E-10 \\
\hline f13 & $1.09 \mathrm{E}-01$ & $7.56 \mathrm{E}-04$ & $1.01 \mathrm{E}-18$ & $1.10 \mathrm{E}-14$ & $1.31 \mathrm{E}-13$ & $2.80 \mathrm{E}-12$ & $3.66 \mathrm{E}-11$ & $2.16 \mathrm{E}-10$ & $1.24 \mathrm{E}-09$ & $1.96 \mathrm{E}-09$ & $5.43 \mathrm{E}-09$ \\
\hline f14 & $1.43 \mathrm{E}+00$ & $1.84 \mathrm{E}+00$ & $1.22 \mathrm{E}+00$ & $9.66 \mathrm{E}-01$ & $5.96 \mathrm{E}-01$ & $7.08 \mathrm{E}-01$ & $7.36 \mathrm{E}-01$ & $8.51 \mathrm{E}-01$ & $1.19 \mathrm{E}+00$ & $1.34 \mathrm{E}+00$ & $1.97 \mathrm{E}+00$ \\
\hline $\mathrm{f} 15$ & $2.62 \mathrm{E}-03$ & $2.04 \mathrm{E}-03$ & $1.39 \mathrm{E}-03$ & $1.45 \mathrm{E}-03$ & $8.45 \mathrm{E}-04$ & $8.33 \mathrm{E}-04$ & $6.24 \mathrm{E}-04$ & $6.31 \mathrm{E}-04$ & $6.24 \mathrm{E}-04$ & $1.19 \mathrm{E}-03$ & $9.47 \mathrm{E}-04$ \\
\hline f16 & $2.35 \mathrm{E}-02$ & $7.68 \mathrm{E}-03$ & $1.60 \mathrm{E}-03$ & $4.56 \mathrm{E}-04$ & $1.57 \mathrm{E}-04$ & $1.26 \mathrm{E}-04$ & $2.05 \mathrm{E}-04$ & $3.01 \mathrm{E}-05$ & $2.14 \mathrm{E}-05$ & $5.36 \mathrm{E}-05$ & $4.27 \mathrm{E}-04$ \\
\hline f17 & $1.19 \mathrm{E}-02$ & $3.29 \mathrm{E}-03$ & 3.75E-03 & $2.40 \mathrm{E}-03$ & $1.19 \mathrm{E}-03$ & $1.57 \mathrm{E}-03$ & $8.64 \mathrm{E}-04$ & $8.04 \mathrm{E}-04$ & $1.20 \mathrm{E}-03$ & $1.43 \mathrm{E}-03$ & $1.99 \mathrm{E}-03$ \\
\hline f18 & $2.12 \mathrm{E}+00$ & $3.06 \mathrm{E}-01$ & $6.95 \mathrm{E}-02$ & $2.09 \mathrm{E}-02$ & $1.02 \mathrm{E}-01$ & $2.27 \mathrm{E}-03$ & $1.34 \mathrm{E}-03$ & $4.14 \mathrm{E}-05$ & 3.67E-04 & $8.27 \mathrm{E}-05$ & $5.28 \mathrm{E}-02$ \\
\hline f19 & $7.51 \mathrm{E}-03$ & $9.95 \mathrm{E}-04$ & 2.23E-04 & $1.39 \mathrm{E}-04$ & $6.28 \mathrm{E}-05$ & $1.03 \mathrm{E}-04$ & 7.60E-05 & $5.68 \mathrm{E}-05$ & $1.17 \mathrm{E}-04$ & 7.46E-05 & $1.99 \mathrm{E}-04$ \\
\hline $\mathrm{f} 20$ & $4.97 \mathrm{E}-02$ & $2.44 \mathrm{E}-02$ & 3.97E-02 & $2.38 \mathrm{E}-02$ & $5.55 \mathrm{E}-02$ & 3.17E-02 & 3.96E-02 & $2.38 \mathrm{E}-02$ & $2.77 \mathrm{E}-02$ & $1.59 \mathrm{E}-02$ & $2.01 \mathrm{E}-02$ \\
\hline $\mathrm{f} 21$ & $5.36 \mathrm{E}+00$ & $3.93 \mathrm{E}+00$ & $3.09 \mathrm{E}+00$ & $2.76 \mathrm{E}+00$ & $1.75 \mathrm{E}+00$ & $2.26 \mathrm{E}+00$ & $1.58 \mathrm{E}+00$ & $1.50 \mathrm{E}+00$ & $7.58 \mathrm{E}-01$ & 2.62E-01 & $5.44 \mathrm{E}-01$ \\
\hline $\mathrm{f} 22$ & $4.50 \mathrm{E}+00$ & $3.01 \mathrm{E}+00$ & $1.33 \mathrm{E}+00$ & $2.04 \mathrm{E}+00$ & $8.92 \mathrm{E}-01$ & $1.43 \mathrm{E}+00$ & $1.67 \mathrm{E}+00$ & $7.00 \mathrm{E}-01$ & $8.32 \mathrm{E}-01$ & $4.65 \mathrm{E}-01$ & $1.03 \mathrm{E}+00$ \\
\hline $\mathrm{f} 23$ & $4.91 \mathrm{E}+00$ & $2.66 \mathrm{E}+00$ & $2.11 \mathrm{E}+00$ & 4.80E-01 & $9.73 \mathrm{E}-01$ & $1.01 \mathrm{E}+00$ & $1.15 \mathrm{E}+00$ & $2.70 \mathrm{E}-01$ & $6.59 \mathrm{E}-01$ & $2.55 \mathrm{E}-01$ & $1.01 \mathrm{E}+00$ \\
\hline F01 & $5.71 \mathrm{E}-01$ & $2.39 \mathrm{E}-18$ & $8.21 \mathrm{E}-22$ & $1.48 \mathrm{E}-16$ & $8.41 \mathrm{E}-14$ & $3.59 \mathrm{E}-12$ & $4.07 \mathrm{E}-11$ & $2.36 \mathrm{E}-10$ & $8.71 \mathrm{E}-10$ & $2.63 \mathrm{E}-09$ & $6.14 \mathrm{E}-09$ \\
\hline F02 & $6.88 \mathrm{E}+03$ & $2.87 \mathrm{E}+03$ & $1.12 \mathrm{E}+03$ & $8.22 \mathrm{E}+02$ & $5.38 \mathrm{E}+02$ & $3.37 \mathrm{E}+02$ & $2.41 \mathrm{E}+02$ & $1.36 \mathrm{E}+02$ & $1.27 \mathrm{E}+02$ & $7.94 \mathrm{E}+01$ & $7.09 \mathrm{E}+01$ \\
\hline F03 & $1.65 \mathrm{E}+07$ & $6.22 \mathrm{E}+06$ & $4.81 \mathrm{E}+06$ & $3.54 \mathrm{E}+06$ & $3.09 \mathrm{E}+06$ & $2.78 \mathrm{E}+06$ & $2.69 \mathrm{E}+06$ & $2.72 \mathrm{E}+06$ & $2.37 \mathrm{E}+06$ & $2.18 \mathrm{E}+06$ & $3.26 \mathrm{E}+06$ \\
\hline F04 & $1.67 \mathrm{E}+04$ & $7.39 \mathrm{E}+03$ & $4.44 \mathrm{E}+03$ & $3.03 \mathrm{E}+03$ & $2.40 \mathrm{E}+03$ & $1.94 \mathrm{E}+03$ & $1.50 \mathrm{E}+03$ & $9.78 \mathrm{E}+02$ & $9.37 \mathrm{E}+02$ & $8.13 \mathrm{E}+02$ & $7.08 \mathrm{E}+02$ \\
\hline F05 & $6.23 \mathrm{E}+03$ & $4.20 \mathrm{E}+03$ & $3.91 \mathrm{E}+03$ & $4.14 \mathrm{E}+03$ & $4.56 \mathrm{E}+03$ & $4.54 \mathrm{E}+03$ & $4.97 \mathrm{E}+03$ & $5.35 \mathrm{E}+03$ & $6.00 \mathrm{E}+03$ & $7.94 \mathrm{E}+03$ & $1.06 \mathrm{E}+04$ \\
\hline F06 & $8.72 \mathrm{E}+02$ & $1.66 \mathrm{E}+03$ & $5.85 \mathrm{E}+02$ & $6.04 \mathrm{E}+02$ & $4.61 \mathrm{E}+02$ & $5.40 \mathrm{E}+02$ & $3.97 \mathrm{E}+02$ & $3.45 \mathrm{E}+02$ & $2.02 \mathrm{E}+02$ & $3.74 \mathrm{E}+02$ & $3.45 \mathrm{E}+02$ \\
\hline F07 & $5.34 \mathrm{E}+03$ & $8.76 \mathrm{E}-01$ & $3.54 \mathrm{E}-01$ & $4.55 \mathrm{E}+00$ & $7.61 \mathrm{E}+01$ & $3.71 \mathrm{E}+02$ & $6.06 \mathrm{E}+02$ & $9.35 \mathrm{E}+02$ & $1.26 \mathrm{E}+03$ & $1.58 \mathrm{E}+03$ & $2.53 \mathrm{E}+03$ \\
\hline F08 & $2.09 \mathrm{E}+01$ & $2.08 \mathrm{E}+01$ & $2.09 \mathrm{E}+01$ & $2.09 \mathrm{E}+01$ & $2.09 \mathrm{E}+01$ & $2.09 \mathrm{E}+01$ & $2.09 \mathrm{E}+01$ & $2.09 \mathrm{E}+01$ & $2.09 \mathrm{E}+01$ & $2.09 \mathrm{E}+01$ & $2.09 \mathrm{E}+01$ \\
\hline F09 & $2.86 \mathrm{E}-01$ & $5.67 \mathrm{E}-11$ & $0.00 \mathrm{E}+00$ & $0.00 \mathrm{E}+00$ & $1.34 \mathrm{E}-13$ & $1.49 \mathrm{E}-11$ & $9.71 \mathrm{E}-11$ & $1.08 \mathrm{E}-09$ & $3.58 \mathrm{E}-09$ & $1.47 \mathrm{E}-08$ & $1.32 \mathrm{E}-07$ \\
\hline F10 & $5.12 \mathrm{E}+01$ & $4.49 \mathrm{E}+01$ & $4.49 \mathrm{E}+01$ & $4.09 \mathrm{E}+01$ & $4.62 \mathrm{E}+01$ & $4.70 \mathrm{E}+01$ & $5.53 \mathrm{E}+01$ & $5.50 \mathrm{E}+01$ & $6.01 \mathrm{E}+01$ & $7.57 \mathrm{E}+01$ & $1.54 \mathrm{E}+02$ \\
\hline F11 & $3.23 \mathrm{E}+01$ & $2.08 \mathrm{E}+01$ & $1.73 \mathrm{E}+01$ & $1.57 \mathrm{E}+01$ & $1.53 \mathrm{E}+01$ & $1.59 \mathrm{E}+01$ & $1.50 \mathrm{E}+01$ & $1.51 \mathrm{E}+01$ & $1.55 \mathrm{E}+01$ & $1.69 \mathrm{E}+01$ & $1.77 \mathrm{E}+01$ \\
\hline F12 & $1.66 \mathrm{E}+00$ & $1.15 \mathrm{E}+04$ & $1.16 \mathrm{E}+04$ & $9.67 \mathrm{E}+03$ & $7.33 \mathrm{E}+03$ & $7.60 \mathrm{E}+03$ & $8.37 \mathrm{E}+03$ & $7.15 \mathrm{E}+03$ & $9.86 \mathrm{E}+03$ & $8.23 \mathrm{E}+03$ & $1.01 \mathrm{E}+04$ \\
\hline F13 & $1.26 \mathrm{E}+00$ & $1.24 \mathrm{E}+00$ & $1.15 \mathrm{E}+00$ & $1.19 \mathrm{E}+00$ & $1.14 \mathrm{E}+00$ & $1.14 \mathrm{E}+00$ & $1.19 \mathrm{E}+00$ & $1.18 \mathrm{E}+00$ & $1.27 \mathrm{E}+00$ & $1.24 \mathrm{E}+00$ & $1.30 \mathrm{E}+00$ \\
\hline F14 & $1.32 \mathrm{E}+01$ & $1.28 \mathrm{E}+01$ & $1.27 \mathrm{E}+01$ & $1.26 \mathrm{E}+01$ & $1.27 \mathrm{E}+01$ & $1.26 \mathrm{E}+01$ & $1.27 \mathrm{E}+01$ & $1.26 \mathrm{E}+01$ & $1.24 \mathrm{E}+01$ & $1.26 \mathrm{E}+01$ & $1.27 \mathrm{E}+01$ \\
\hline
\end{tabular}

The mean error is recorded based on 30 independent runs, and the best results are highlighted in boldface 
Table A-2 Mean error of CMM-rcBBOg with different $P_{e}$ on the 37 functions

\begin{tabular}{|c|c|c|c|c|c|c|c|c|c|c|c|}
\hline$P_{e}$ & 0.0 & 0.1 & 0.2 & 0.3 & 0.4 & 0.5 & 0.6 & 0.7 & 0.8 & 0.9 & 1.0 \\
\hline f01 & $5.26 \mathrm{E}-04$ & $3.53 \mathrm{E}-11$ & $4.05 \mathrm{E}-23$ & 8.83E-19 & $1.85 \mathrm{E}-16$ & $4.81 \mathrm{E}-15$ & $6.20 \mathrm{E}-14$ & $2.49 \mathrm{E}-13$ & $1.06 \mathrm{E}-12$ & $2.41 \mathrm{E}-12$ & $8.60 \mathrm{E}-12$ \\
\hline f02 & $5.14 \mathrm{E}-02$ & $8.29 \mathrm{E}-08$ & $4.75 \mathrm{E}-13$ & $3.15 \mathrm{E}-10$ & $1.08 \mathrm{E}-08$ & $7.98 \mathrm{E}-08$ & $2.84 \mathrm{E}-07$ & $7.89 \mathrm{E}-07$ & $1.55 \mathrm{E}-06$ & $3.12 \mathrm{E}-06$ & 5.49E-06 \\
\hline f03 & $2.32 \mathrm{E}+01$ & $7.74 \mathrm{E}+00$ & $3.70 \mathrm{E}+00$ & $2.38 \mathrm{E}+00$ & $1.48 \mathrm{E}+00$ & $1.16 \mathrm{E}+00$ & $6.67 \mathrm{E}-01$ & $3.40 \mathrm{E}-01$ & $2.03 \mathrm{E}-01$ & $1.83 \mathrm{E}-01$ & $1.25 \mathrm{E}-01$ \\
\hline f04 & $6.49 \mathrm{E}-02$ & 2.43E-02 & $1.40 \mathrm{E}-02$ & $1.23 \mathrm{E}-02$ & $9.74 \mathrm{E}-03$ & $1.12 \mathrm{E}-02$ & $1.17 \mathrm{E}-02$ & $1.41 \mathrm{E}-02$ & $1.90 \mathrm{E}-02$ & $1.87 \mathrm{E}-02$ & 3.35E-02 \\
\hline f05 & $9.31 \mathrm{E}+01$ & $1.04 \mathrm{E}+02$ & $5.23 \mathrm{E}+01$ & $4.40 \mathrm{E}+01$ & $3.98 \mathrm{E}+01$ & $3.44 \mathrm{E}+01$ & $2.99 \mathrm{E}+01$ & $3.07 \mathrm{E}+01$ & $3.27 \mathrm{E}+01$ & $3.19 \mathrm{E}+01$ & $4.34 \mathrm{E}+01$ \\
\hline f06 & $0.00 \mathrm{E}+00$ & $0.00 \mathrm{E}+00$ & $0.00 \mathrm{E}+00$ & $0.00 \mathrm{E}+00$ & $0.00 \mathrm{E}+00$ & $0.00 \mathrm{E}+00$ & $0.00 \mathrm{E}+00$ & $0.00 \mathrm{E}+00$ & $0.00 \mathrm{E}+00$ & $0.00 \mathrm{E}+00$ & $0.00 \mathrm{E}+00$ \\
\hline f07 & $4.87 \mathrm{E}-03$ & $1.06 \mathrm{E}-03$ & $1.35 \mathrm{E}-03$ & $1.75 \mathrm{E}-03$ & $1.73 \mathrm{E}-03$ & $2.08 \mathrm{E}-03$ & $2.36 \mathrm{E}-03$ & $2.42 \mathrm{E}-03$ & $2.53 \mathrm{E}-03$ & $2.90 \mathrm{E}-03$ & 2.97E-03 \\
\hline $\mathrm{f} 08$ & $4.76 \mathrm{E}+02$ & $4.72 \mathrm{E}+02$ & $6.30 \mathrm{E}+02$ & $1.16 \mathrm{E}+03$ & $2.26 \mathrm{E}+03$ & $3.14 \mathrm{E}+03$ & $4.19 \mathrm{E}+03$ & $4.46 \mathrm{E}+03$ & $4.80 \mathrm{E}+03$ & $4.99 \mathrm{E}+03$ & $4.82 \mathrm{E}+03$ \\
\hline f09 & $1.44 \mathrm{E}-02$ & $4.09 \mathrm{E}-15$ & $0.00 \mathrm{E}+00$ & $0.00 \mathrm{E}+00$ & $2.72 \mathrm{E}-15$ & $1.22 \mathrm{E}-13$ & $1.72 \mathrm{E}-12$ & $1.18 \mathrm{E}-11$ & $4.97 \mathrm{E}-11$ & $1.85 \mathrm{E}-10$ & $4.71 \mathrm{E}-10$ \\
\hline f10 & $1.53 \mathrm{E}-02$ & $2.27 \mathrm{E}-06$ & $2.20 \mathrm{E}-12$ & $4.28 \mathrm{E}-10$ & 7.74E-09 & $4.35 \mathrm{E}-08$ & $1.45 \mathrm{E}-07$ & 3.34E-07 & $6.79 \mathrm{E}-07$ & $1.13 \mathrm{E}-06$ & 2.14E-06 \\
\hline f11 & $2.99 \mathrm{E}-01$ & $6.06 \mathrm{E}-03$ & $1.89 \mathrm{E}-03$ & 5.75E-04 & $2.05 \mathrm{E}-03$ & $2.05 \mathrm{E}-03$ & $1.88 \mathrm{E}-03$ & 7.87E-03 & $1.90 \mathrm{E}-02$ & $2.35 \mathrm{E}-02$ & $9.28 \mathrm{E}-03$ \\
\hline f12 & $6.26 \mathrm{E}-01$ & $5.19 \mathrm{E}-02$ & $9.17 \mathrm{E}-25$ & $3.46 \mathrm{E}-03$ & $6.91 \mathrm{E}-03$ & 4.03E-17 & $1.73 \mathrm{E}-02$ & $6.91 \mathrm{E}-03$ & $1.38 \mathrm{E}-02$ & $1.04 \mathrm{E}-02$ & 7.95E-02 \\
\hline f13 & $1.18 \mathrm{E}-04$ & 1.12E-10 & $9.35 \mathrm{E}-23$ & $1.59 \mathrm{E}-19$ & 4.99E-17 & $1.14 \mathrm{E}-15$ & $1.38 \mathrm{E}-14$ & $6.70 \mathrm{E}-14$ & $2.66 \mathrm{E}-13$ & $1.27 \mathrm{E}-12$ & 3.56E-12 \\
\hline f14 & $3.02 \mathrm{E}+00$ & $2.44 \mathrm{E}+00$ & $1.43 \mathrm{E}+00$ & $1.12 \mathrm{E}+00$ & $1.07 \mathrm{E}+00$ & $1.59 \mathrm{E}+00$ & $1.39 \mathrm{E}+00$ & $1.04 \mathrm{E}+00$ & $1.49 \mathrm{E}+00$ & $1.38 \mathrm{E}+00$ & $2.99 \mathrm{E}+00$ \\
\hline $\mathrm{f} 15$ & $4.11 \mathrm{E}-03$ & $1.21 \mathrm{E}-03$ & 7.82E-04 & 7.96E-04 & $5.21 \mathrm{E}-04$ & $6.88 \mathrm{E}-04$ & $5.85 \mathrm{E}-04$ & $5.64 \mathrm{E}-04$ & $5.43 \mathrm{E}-04$ & $6.04 \mathrm{E}-04$ & 7.03E- 04 \\
\hline f16 & $1.13 \mathrm{E}-02$ & $2.73 \mathrm{E}-03$ & $1.28 \mathrm{E}-03$ & $9.06 \mathrm{E}-04$ & 7.60E-04 & $1.84 \mathrm{E}-04$ & $1.31 \mathrm{E}-04$ & $2.88 \mathrm{E}-05$ & $1.82 E-05$ & $3.54 \mathrm{E}-05$ & $1.17 \mathrm{E}-04$ \\
\hline f17 & $8.76 \mathrm{E}-03$ & $3.56 \mathrm{E}-03$ & $2.58 \mathrm{E}-03$ & $1.39 \mathrm{E}-03$ & $5.75 \mathrm{E}-04$ & $1.08 \mathrm{E}-03$ & $1.62 \mathrm{E}-03$ & $1.71 \mathrm{E}-03$ & $1.15 \mathrm{E}-03$ & $9.94 \mathrm{E}-04$ & $1.67 \mathrm{E}-03$ \\
\hline f18 & $1.34 \mathrm{E}+00$ & $1.72 \mathrm{E}-01$ & $4.74 \mathrm{E}-02$ & $1.47 \mathrm{E}-02$ & $4.05 \mathrm{E}-02$ & 2.13E-02 & $2.83 \mathrm{E}-01$ & $1.64 \mathrm{E}-03$ & $4.27 \mathrm{E}-06$ & $1.15 \mathrm{E}-05$ & 3.16E-02 \\
\hline f19 & $1.21 \mathrm{E}-02$ & $1.28 \mathrm{E}-03$ & $1.99 \mathrm{E}-04$ & $8.37 \mathrm{E}-05$ & 6.88E-05 & $1.17 \mathrm{E}-04$ & $1.08 \mathrm{E}-04$ & $1.35 \mathrm{E}-04$ & $1.07 \mathrm{E}-04$ & $5.68 \mathrm{E}-04$ & $8.30 \mathrm{E}-05$ \\
\hline $\mathrm{f} 20$ & $5.02 \mathrm{E}-02$ & $2.83 \mathrm{E}-02$ & $3.57 \mathrm{E}-02$ & $2.38 \mathrm{E}-02$ & $2.78 \mathrm{E}-02$ & $1.98 \mathrm{E}-02$ & $3.57 \mathrm{E}-02$ & $1.98 \mathrm{E}-02$ & $1.98 \mathrm{E}-02$ & $2.38 \mathrm{E}-02$ & $1.60 \mathrm{E}-02$ \\
\hline $\mathrm{f} 21$ & $3.91 \mathrm{E}+00$ & $3.20 \mathrm{E}+00$ & $4.33 \mathrm{E}+00$ & $2.51 \mathrm{E}+00$ & $3.08 \mathrm{E}+00$ & $1.83 \mathrm{E}+00$ & $1.58 \mathrm{E}+00$ & $4.41 \mathrm{E}-01$ & $1.08 \mathrm{E}+00$ & 7.49E-01 & $5.76 \mathrm{E}-01$ \\
\hline $\mathrm{f} 22$ & $3.07 \mathrm{E}+00$ & $2.93 \mathrm{E}+00$ & $1.46 \mathrm{E}+00$ & $1.91 \mathrm{E}+00$ & $2.07 \mathrm{E}+00$ & $1.32 \mathrm{E}+00$ & $1.81 \mathrm{E}+00$ & $6.21 \mathrm{E}-01$ & $4.78 \mathrm{E}-01$ & 4.64E-01 & $2.84 \mathrm{E}-01$ \\
\hline $\mathrm{f} 23$ & $3.51 \mathrm{E}+00$ & $1.21 \mathrm{E}+00$ & $1.39 \mathrm{E}+00$ & $1.63 \mathrm{E}+00$ & $4.79 \mathrm{E}-01$ & $9.73 \mathrm{E}-01$ & $5.26 \mathrm{E}-01$ & $4.79 \mathrm{E}-01$ & $2.84 \mathrm{E}-01$ & $2.55 \mathrm{E}-01$ & 1.80E-01 \\
\hline F01 & $8.94 \mathrm{E}-05$ & $5.49 \mathrm{E}-22$ & 4.24E-26 & $2.53 \mathrm{E}-20$ & $1.57 \mathrm{E}-17$ & $4.73 \mathrm{E}-16$ & $5.78 \mathrm{E}-15$ & $3.86 \mathrm{E}-14$ & $1.65 \mathrm{E}-13$ & $6.04 \mathrm{E}-13$ & $6.26 \mathrm{E}-12$ \\
\hline F02 & $1.88 \mathrm{E}+02$ & $7.78 \mathrm{E}+01$ & $4.91 \mathrm{E}+01$ & $3.74 \mathrm{E}+01$ & $3.71 \mathrm{E}+01$ & $3.67 \mathrm{E}+01$ & $3.26 \mathrm{E}+01$ & $3.69 \mathrm{E}+01$ & $4.47 \mathrm{E}+01$ & $8.61 \mathrm{E}+01$ & $5.35 \mathrm{E}+02$ \\
\hline F03 & $3.44 \mathrm{E}+06$ & $2.56 \mathrm{E}+06$ & $2.31 \mathrm{E}+00$ & $1.90 \mathrm{E}+06$ & $1.70 \mathrm{E}+06$ & $1.71 \mathrm{E}+06$ & $1.60 \mathrm{E}+06$ & $1.62 \mathrm{E}+06$ & $1.98 \mathrm{E}+06$ & $1.93 \mathrm{E}+06$ & $3.26 \mathrm{E}+06$ \\
\hline F04 & $2.02 \mathrm{E}+04$ & $7.00 \mathrm{E}+03$ & $7.76 \mathrm{E}+03$ & $5.38 \mathrm{E}+03$ & $5.50 \mathrm{E}+03$ & $5.04 E+03$ & $5.89 \mathrm{E}+03$ & $5.41 \mathrm{E}+03$ & $5.54 \mathrm{E}+03$ & $6.41 \mathrm{E}+03$ & $1.16 \mathrm{E}+04$ \\
\hline F05 & $6.42 \mathrm{E}+03$ & $4.37 \mathrm{E}+03$ & $4.49 \mathrm{E}+03$ & $4.61 \mathrm{E}+03$ & $4.77 \mathrm{E}+03$ & $5.02 \mathrm{E}+03$ & $5.70 \mathrm{E}+03$ & $6.58 \mathrm{E}+03$ & $7.50 \mathrm{E}+03$ & $9.75 \mathrm{E}+03$ & $1.52 \mathrm{E}+04$ \\
\hline F06 & $4.11 \mathrm{E}+03$ & $1.30 \mathrm{E}+03$ & $4.47 \mathrm{E}+02$ & $4.59 \mathrm{E}+02$ & $6.27 \mathrm{E}+02$ & $2.82 \mathrm{E}+02$ & $1.03 \mathrm{E}+03$ & $5.99 \mathrm{E}+02$ & $6.30 \mathrm{E}+02$ & $1.03 \mathrm{E}+03$ & $8.43 \mathrm{E}+02$ \\
\hline F07 & $2.24 \mathrm{E}+03$ & $5.98 \mathrm{E}+02$ & $3.04 \mathrm{E}+02$ & $2.19 \mathrm{E}+02$ & $2.18 \mathrm{E}+02$ & $2.50 \mathrm{E}+02$ & $3.01 \mathrm{E}+02$ & $4.23 \mathrm{E}+02$ & $7.28 \mathrm{E}+02$ & $1.52 \mathrm{E}+03$ & $6.60 \mathrm{E}+03$ \\
\hline F08 & $2.07 \mathrm{E}+01$ & $2.06 \mathrm{E}+01$ & $2.06 \mathrm{E}+01$ & $2.06 \mathrm{E}+01$ & $2.06 \mathrm{E}+01$ & $2.06 \mathrm{E}+01$ & $2.07 \mathrm{E}+01$ & $2.06 \mathrm{E}+01$ & $2.07 \mathrm{E}+01$ & $2.06 \mathrm{E}+01$ & $2.07 \mathrm{E}+01$ \\
\hline F09 & $1.76 \mathrm{E}-02$ & $1.15 \mathrm{E}-14$ & $0.00 \mathrm{E}+00$ & $0.00 \mathrm{E}+00$ & $3.02 \mathrm{E}-15$ & $1.08 \mathrm{E}-13$ & $1.91 \mathrm{E}-12$ & $1.27 \mathrm{E}-11$ & $5.37 \mathrm{E}-11$ & $1.65 \mathrm{E}-10$ & $5.42 \mathrm{E}-10$ \\
\hline F10 & $5.92 \mathrm{E}+01$ & $4.85 \mathrm{E}+01$ & $4.69 \mathrm{E}+01$ & $4.69 \mathrm{E}+01$ & $4.52 \mathrm{E}+01$ & $5.05 \mathrm{E}+01$ & $4.99 \mathrm{E}+01$ & $6.71 \mathrm{E}+01$ & $6.85 \mathrm{E}+01$ & $9.32 \mathrm{E}+01$ & $1.83 \mathrm{E}+02$ \\
\hline F11 & $3.13 \mathrm{E}+01$ & $1.93 \mathrm{E}+01$ & $1.68 \mathrm{E}+01$ & $1.57 \mathrm{E}+01$ & $1.53 \mathrm{E}+01$ & $1.51 \mathrm{E}+01$ & $1.52 \mathrm{E}+01$ & $1.46 \mathrm{E}+01$ & $1.49 \mathrm{E}+01$ & $1.64 \mathrm{E}+01$ & $1.89 \mathrm{E}+01$ \\
\hline F12 & $1.95 \mathrm{E}+04$ & $1.57 \mathrm{E}+04$ & $1.08 \mathrm{E}+04$ & $1.29 \mathrm{E}+04$ & $1.09 \mathrm{E}+04$ & $1.03 E+00$ & $7.94 \mathrm{E}+03$ & $9.72 \mathrm{E}+03$ & $1.12 \mathrm{E}+04$ & $1.28 \mathrm{E}+04$ & $1.42 \mathrm{E}+04$ \\
\hline F13 & $1.24 \mathrm{E}+00$ & $1.06 \mathrm{E}+00$ & $1.10 \mathrm{E}+00$ & $1.06 \mathrm{E}+00$ & $1.07 \mathrm{E}+00$ & $1.11 \mathrm{E}+00$ & $1.10 \mathrm{E}+00$ & $1.18 \mathrm{E}+00$ & $1.07 \mathrm{E}+00$ & $1.21 \mathrm{E}+00$ & $1.31 \mathrm{E}+00$ \\
\hline F14 & $1.36 \mathrm{E}+01$ & $1.32 \mathrm{E}+01$ & $1.31 \mathrm{E}+01$ & $1.32 \mathrm{E}+01$ & $1.32 \mathrm{E}+01$ & $1.30 \mathrm{E}+01$ & $1.31 \mathrm{E}+01$ & $1.30 \mathrm{E}+01$ & $1.30 \mathrm{E}+01$ & $1.30 \mathrm{E}+01$ & $1.31 \mathrm{E}+01$ \\
\hline
\end{tabular}

The mean error is recorded based on 30 independent runs, and the best results are highlighted in boldface 
Table A-3 Mean errors of CMM-pBBO with different $P_{e}$ on the 37 functions

\begin{tabular}{|c|c|c|c|c|c|c|c|c|c|c|c|}
\hline$P_{e}$ & 0.0 & 0.1 & 0.2 & 0.3 & 0.4 & 0.5 & 0.6 & 0.7 & 0.8 & 0.9 & 1.0 \\
\hline f01 & $7.74 \mathrm{E}-08$ & $1.34 \mathrm{E}-24$ & $3.95 \mathrm{E}-16$ & $2.31 \mathrm{E}-13$ & $8.04 \mathrm{E}-12$ & $6.31 \mathrm{E}-11$ & $2.84 \mathrm{E}-10$ & $8.87 \mathrm{E}-10$ & $1.93 \mathrm{E}-09$ & $3.94 \mathrm{E}-09$ & $8.19 \mathrm{E}-09$ \\
\hline $\mathrm{f} 02$ & $3.32 \mathrm{E}-05$ & 3.33E-14 & 7.64E-09 & $3.29 \mathrm{E}-07$ & $2.30 \mathrm{E}-06$ & 7.98E-06 & 2.06E-05 & $4.14 \mathrm{E}-05$ & $6.95 \mathrm{E}-05$ & $1.08 \mathrm{E}-04$ & $1.49 \mathrm{E}-04$ \\
\hline f03 & $2.15 \mathrm{E}-01$ & $2.87 \mathrm{E}-03$ & $3.00 \mathrm{E}-04$ & $1.63 \mathrm{E}-05$ & 7.67E-07 & $2.29 \mathrm{E}-07$ & 3.03E-07 & $5.80 \mathrm{E}-07$ & $8.63 \mathrm{E}-07$ & $1.51 \mathrm{E}-06$ & $2.00 \mathrm{E}-06$ \\
\hline f04 & 7.87E-03 & $1.32 \mathrm{E}-06$ & $4.45 \mathrm{E}-08$ & 6.53E-07 & $2.88 \mathrm{E}-06$ & $7.50 \mathrm{E}-06$ & $1.36 \mathrm{E}-05$ & $2.40 \mathrm{E}-05$ & $3.59 \mathrm{E}-05$ & $4.91 \mathrm{E}-05$ & $6.54 \mathrm{E}-05$ \\
\hline f05 & $4.55 \mathrm{E}+01$ & $3.94 \mathrm{E}+01$ & $4.83 \mathrm{E}+01$ & $3.65 \mathrm{E}+01$ & $3.77 \mathrm{E}+01$ & $2.50 \mathrm{E}+01$ & $2.87 \mathrm{E}+01$ & $2.95 \mathrm{E}+01$ & $2.25 \mathrm{E}+01$ & $2.57 \mathrm{E}+01$ & $3.21 \mathrm{E}+01$ \\
\hline f06 & $0.00 \mathrm{E}+00$ & $0.00 \mathrm{E}+00$ & $0.00 \mathrm{E}+00$ & $0.00 \mathrm{E}+00$ & $0.00 \mathrm{E}+00$ & $0.00 \mathrm{E}+00$ & $0.00 \mathrm{E}+00$ & $0.00 \mathrm{E}+00$ & $0.00 \mathrm{E}+00$ & $0.00 \mathrm{E}+00$ & $0.00 \mathrm{E}+00$ \\
\hline f07 & $2.04 \mathrm{E}-03$ & $5.13 \mathrm{E}-03$ & $6.54 \mathrm{E}-03$ & $7.48 \mathrm{E}-03$ & $7.92 \mathrm{E}-03$ & $9.15 \mathrm{E}-03$ & $9.40 \mathrm{E}-03$ & $8.86 \mathrm{E}-03$ & $8.89 \mathrm{E}-03$ & $8.30 \mathrm{E}-03$ & $6.89 \mathrm{E}-03$ \\
\hline $\mathrm{f} 08$ & $3.63 \mathrm{E}+02$ & $4.48 \mathrm{E}+02$ & $6.19 \mathrm{E}+02$ & $8.47 \mathrm{E}+02$ & $1.45 \mathrm{E}+03$ & $1.97 \mathrm{E}+03$ & $2.62 \mathrm{E}+03$ & $3.11 \mathrm{E}+03$ & $3.62 \mathrm{E}+03$ & $4.27 \mathrm{E}+03$ & $4.88 \mathrm{E}+03$ \\
\hline f09 & $4.30 \mathrm{E}-07$ & $0.00 \mathrm{E}+00$ & $4.74 \mathrm{E}-16$ & $7.42 \mathrm{E}-13$ & $3.08 \mathrm{E}-11$ & $4.93 \mathrm{E}-10$ & 3.05E-09 & $1.15 \mathrm{E}-08$ & $2.87 \mathrm{E}-08$ & $6.92 \mathrm{E}-08$ & $1.28 \mathrm{E}-07$ \\
\hline $\mathrm{f} 10$ & 3.32E-05 & $5.90 \mathrm{E}-13$ & $1.21 \mathrm{E}-08$ & $3.27 \mathrm{E}-07$ & $1.90 \mathrm{E}-06$ & $5.45 \mathrm{E}-06$ & $1.14 \mathrm{E}-05$ & $1.95 \mathrm{E}-05$ & $2.84 \mathrm{E}-05$ & 4.04E- 05 & $6.73 \mathrm{E}-05$ \\
\hline f1 1 & $1.45 \mathrm{E}-02$ & $1.56 \mathrm{E}-03$ & $6.57 \mathrm{E}-04$ & $2.60 \mathrm{E}-15$ & 4.93E-04 & $3.29 \mathrm{E}-04$ & $1.40 \mathrm{E}-03$ & $3.37 \mathrm{E}-03$ & $5.50 \mathrm{E}-03$ & $2.35 \mathrm{E}-02$ & $9.60 \mathrm{E}-03$ \\
\hline f12 & 4.49E-02 & $1.04 \mathrm{E}-02$ & $2.07 \mathrm{E}-02$ & $1.81 \mathrm{E}-15$ & $1.04 \mathrm{E}-02$ & $3.46 \mathrm{E}-03$ & $1.73 \mathrm{E}-02$ & $3.46 \mathrm{E}-03$ & $2.07 \mathrm{E}-02$ & $3.11 \mathrm{E}-02$ & $3.80 \mathrm{E}-02$ \\
\hline $\mathrm{f} 13$ & $8.01 \mathrm{E}-09$ & $1.95 \mathrm{E}-25$ & $4.99 \mathrm{E}-17$ & 4.47E-14 & $1.17 \mathrm{E}-12$ & $1.14 \mathrm{E}-11$ & $6.95 \mathrm{E}-11$ & $2.07 \mathrm{E}-10$ & $5.22 \mathrm{E}-10$ & $1.20 \mathrm{E}-09$ & 2.60E-09 \\
\hline f14 & $5.93 \mathrm{E}-01$ & $9.21 \mathrm{E}-01$ & $1.48 \mathrm{E}+00$ & $7.02 \mathrm{E}-01$ & $8.77 \mathrm{E}-01$ & $4.61 \mathrm{E}-01$ & $8.00 \mathrm{E}-01$ & $1.22 \mathrm{E}+00$ & $8.34 \mathrm{E}-01$ & $1.50 \mathrm{E}+00$ & $2.27 \mathrm{E}+00$ \\
\hline f15 & $1.35 \mathrm{E}-03$ & $1.15 \mathrm{E}-03$ & $4.33 \mathrm{E}-04$ & 3.94E-04 & $1.07 \mathrm{E}-03$ & $3.52 \mathrm{E}-04$ & $4.45 \mathrm{E}-04$ & $3.64 \mathrm{E}-04$ & 3.64E-04 & $2.87 \mathrm{E}-04$ & $2.82 \mathrm{E}-04$ \\
\hline $\mathrm{f} 16$ & $2.19 \mathrm{E}-04$ & $2.38 \mathrm{E}-15$ & $1.54 \mathrm{E}-14$ & $2.35 \mathrm{E}-15$ & $2.26 \mathrm{E}-15$ & $2.26 \mathrm{E}-15$ & $7.46 \mathrm{E}-09$ & 2.23E-15 & $2.22 \mathrm{E}-15$ & $2.23 \mathrm{E}-15$ & $6.37 \mathrm{E}-08$ \\
\hline f17 & $2.10 \mathrm{E}-05$ & $1.19 \mathrm{E}-05$ & 3.95E-06 & $6.14 \mathrm{E}-06$ & $4.07 \mathrm{E}-06$ & $3.22 \mathrm{E}-06$ & $3.25 \mathrm{E}-07$ & $1.86 \mathrm{E}-07$ & $1.45 \mathrm{E}-09$ & $7.69 \mathrm{E}-08$ & $5.56 \mathrm{E}-06$ \\
\hline f18 & $7.50 \mathrm{E}-03$ & $7.49 \mathrm{E}-06$ & $3.14 \mathrm{E}-15$ & $2.53 \mathrm{E}-09$ & $7.74 \mathrm{E}-14$ & $1.91 \mathrm{E}-15$ & $1.44 \mathrm{E}-15$ & $2.83 \mathrm{E}-15$ & $1.04 \mathrm{E}-15$ & $1.38 \mathrm{E}-15$ & 3.32E-10 \\
\hline f19 & $1.74 \mathrm{E}-06$ & $1.62 \mathrm{E}-06$ & 3.17E-07 & $2.75 \mathrm{E}-07$ & $2.72 \mathrm{E}-07$ & $2.55 \mathrm{E}-07$ & $2.56 \mathrm{E}-07$ & $7.83 \mathrm{E}-07$ & $2.54 \mathrm{E}-07$ & $2.55 \mathrm{E}-07$ & $2.54 \mathrm{E}-05$ \\
\hline $\mathrm{f} 20$ & $4.76 \mathrm{E}-02$ & $3.57 \mathrm{E}-02$ & $3.17 \mathrm{E}-02$ & $2.77 \mathrm{E}-02$ & $2.38 \mathrm{E}-02$ & $2.77 \mathrm{E}-02$ & $2.77 \mathrm{E}-02$ & $1.19 \mathrm{E}-02$ & $2.77 \mathrm{E}-02$ & $1.98 \mathrm{E}-02$ & $1.59 \mathrm{E}-02$ \\
\hline $\mathrm{f} 21$ & $5.09 \mathrm{E}+00$ & $3.83 \mathrm{E}+00$ & $2.84 \mathrm{E}+00$ & $2.51 \mathrm{E}+00$ & $1.42 \mathrm{E}+00$ & $2.75 \mathrm{E}+00$ & $1.42 \mathrm{E}+00$ & $1.33 \mathrm{E}+00$ & $1.34 \mathrm{E}+00$ & $7.58 \mathrm{E}-01$ & 4.19E-01 \\
\hline $\mathrm{f} 22$ & $3.34 \mathrm{E}+00$ & $2.77 \mathrm{E}+00$ & $1.31 \mathrm{E}+00$ & $2.07 \mathrm{E}+00$ & $7.50 \mathrm{E}-01$ & $4.78 \mathrm{E}-01$ & $4.78 \mathrm{E}-01$ & 4.45E-01 & $-3.18 \mathrm{E}-11^{*}$ & $4.77 \mathrm{E}-01$ & $4.77 \mathrm{E}-01$ \\
\hline $\mathrm{f} 23$ & $3.16 \mathrm{E}+00$ & $1.92 \mathrm{E}+00$ & $2.12 \mathrm{E}+00$ & $1.44 \mathrm{E}+00$ & $1.00 \mathrm{E}+00$ & $4.04 \mathrm{E}-01$ & $1.33 \mathrm{E}+00$ & $8.38 \mathrm{E}-01$ & $4.02 \mathrm{E}-01$ & $-9.82 \mathrm{E}-06$ & $2.23 \mathrm{E}-01$ \\
\hline F01 & $6.98 \mathrm{E}-10$ & $2.82 \mathrm{E}-27$ & $9.06 \mathrm{E}-18$ & $6.73 \mathrm{E}-15$ & $4.22 \mathrm{E}-13$ & $4.21 \mathrm{E}-12$ & $2.23 \mathrm{E}-11$ & $7.87 \mathrm{E}-11$ & $1.99 \mathrm{E}-10$ & $4.36 \mathrm{E}-10$ & $2.88 \mathrm{E}-09$ \\
\hline F02 & $4.18 \mathrm{E}+00$ & $4.07 \mathrm{E}-01$ & $1.55 \mathrm{E}-01$ & $6.93 \mathrm{E}-02$ & $3.25 \mathrm{E}-02$ & $1.79 \mathrm{E}-02$ & $7.89 \mathrm{E}-03$ & $3.67 \mathrm{E}-03$ & $7.47 \mathrm{E}-03$ & $1.73 \mathrm{E}-02$ & $3.73 \mathrm{E}+01$ \\
\hline F03 & $2.09 \mathrm{E}+06$ & $1.61 \mathrm{E}+06$ & $1.24 \mathrm{E}+06$ & $1.15 \mathrm{E}+06$ & $1.18 \mathrm{E}+06$ & $1.03 \mathrm{E}+06$ & $9.92 \mathrm{E}+05$ & $1.09 \mathrm{E}+06$ & $1.09 \mathrm{E}+06$ & $1.16 \mathrm{E}+06$ & $1.35 \mathrm{E}+06$ \\
\hline F04 & $9.48 \mathrm{E}+02$ & $7.37 \mathrm{E}+01$ & $1.35 \mathrm{E}+01$ & $4.19 \mathrm{E}+00$ & $7.84 \mathrm{E}-01$ & $3.11 \mathrm{E}-01$ & $9.07 \mathrm{E}-02$ & $6.08 \mathrm{E}-02$ & $5.00 \mathrm{E}-02$ & $5.76 \mathrm{E}-02$ & $2.17 \mathrm{E}+02$ \\
\hline F05 & $4.63 \mathrm{E}+03$ & $3.65 \mathrm{E}+03$ & $3.82 \mathrm{E}+03$ & $3.74 \mathrm{E}+03$ & $3.75 \mathrm{E}+03$ & $4.07 \mathrm{E}+03$ & $4.15 \mathrm{E}+03$ & $4.70 \mathrm{E}+03$ & $5.49 \mathrm{E}+03$ & $6.41 \mathrm{E}+03$ & $1.01 \mathrm{E}+04$ \\
\hline F06 & $6.82 \mathrm{E}+02$ & $6.72 \mathrm{E}+02$ & $3.04 \mathrm{E}+02$ & $3.06 \mathrm{E}+02$ & $2.30 \mathrm{E}+02$ & $3.30 \mathrm{E}+02$ & $3.65 \mathrm{E}+02$ & $1.63 \mathrm{E}+02$ & $5.52 \mathrm{E}+02$ & $4.18 \mathrm{E}+02$ & $8.26 \mathrm{E}+02$ \\
\hline F07 & $1.46 \mathrm{E}-02$ & $1.28 \mathrm{E}-02$ & $1.17 \mathrm{E}-02$ & $1.47 \mathrm{E}-02$ & $1.20 \mathrm{E}-02$ & $1.30 \mathrm{E}-02$ & $1.26 \mathrm{E}-02$ & $2.23 \mathrm{E}-02$ & $2.76 \mathrm{E}-02$ & $1.50 \mathrm{E}+00$ & $5.15 \mathrm{E}+03$ \\
\hline F08 & $2.03 \mathrm{E}+01$ & $2.02 \mathrm{E}+01$ & $2.06 \mathrm{E}+01$ & $2.07 \mathrm{E}+01$ & $2.07 \mathrm{E}+01$ & $2.06 \mathrm{E}+01$ & $2.07 \mathrm{E}+01$ & $2.07 \mathrm{E}+01$ & $2.06 \mathrm{E}+01$ & $2.06 \mathrm{E}+01$ & $2.06 \mathrm{E}+01$ \\
\hline F09 & $8.20 \mathrm{E}-07$ & $0.00 \mathrm{E}+00$ & $2.37 \mathrm{E}-16$ & $6.12 \mathrm{E}-13$ & $2.74 \mathrm{E}-11$ & $3.55 \mathrm{E}-10$ & $2.77 \mathrm{E}-09$ & $1.09 \mathrm{E}-08$ & $3.43 \mathrm{E}-08$ & $8.39 \mathrm{E}-08$ & $1.85 \mathrm{E}-07$ \\
\hline F10 & $5.74 \mathrm{E}+01$ & $5.52 \mathrm{E}+01$ & $5.14 \mathrm{E}+01$ & $4.82 \mathrm{E}+01$ & $4.59 \mathrm{E}+01$ & $5.35 \mathrm{E}+01$ & $5.28 \mathrm{E}+01$ & $5.17 \mathrm{E}+01$ & $5.93 \mathrm{E}+01$ & $7.51 \mathrm{E}+01$ & $1.39 \mathrm{E}+02$ \\
\hline F11 & $2.86 \mathrm{E}+01$ & $1.80 \mathrm{E}+01$ & $1.86 \mathrm{E}+01$ & $1.60 \mathrm{E}+01$ & $1.36 \mathrm{E}+01$ & $1.31 \mathrm{E}+01$ & $1.34 \mathrm{E}+01$ & $1.30 \mathrm{E}+01$ & $1.23 E+01$ & $1.39 \mathrm{E}+01$ & $1.54 \mathrm{E}+01$ \\
\hline F12 & $1.13 \mathrm{E}+04$ & $7.65 \mathrm{E}+03$ & $9.20 \mathrm{E}+03$ & $6.16 \mathrm{E}+03$ & $5.04 \mathrm{E}+03$ & $6.06 \mathrm{E}+03$ & $4.99 \mathrm{E}+03$ & $5.39 \mathrm{E}+03$ & $6.16 \mathrm{E}+03$ & $1.15 \mathrm{E}+04$ & $8.54 \mathrm{E}+03$ \\
\hline F13 & $1.08 \mathrm{E}+00$ & $1.08 \mathrm{E}+00$ & $1.10 \mathrm{E}+00$ & $1.13 \mathrm{E}+00$ & $1.11 \mathrm{E}+00$ & $1.10 \mathrm{E}+00$ & $1.08 \mathrm{E}+00$ & $1.13 \mathrm{E}+00$ & $1.25 \mathrm{E}+00$ & $1.45 \mathrm{E}+00$ & $1.43 \mathrm{E}+00$ \\
\hline F14 & $1.35 \mathrm{E}+01$ & $1.32 \mathrm{E}+01$ & $1.31 \mathrm{E}+01$ & $1.32 \mathrm{E}+01$ & $1.31 \mathrm{E}+01$ & $1.31 \mathrm{E}+01$ & $1.30 \mathrm{E}+01$ & $1.30 \mathrm{E}+01$ & $1.30 \mathrm{E}+01$ & $1.30 \mathrm{E}+01$ & $1.31 \mathrm{E}+01$ \\
\hline
\end{tabular}

The mean error is recorded based on 30 independent runs, and the best results are highlighted in boldface.

* A negative value means that the achieved value is better than the best result provided in Table 1 . 
Table A-4 Mean errors of CMM-DE/BBO with different $P_{e}$ on the 37 functions

\begin{tabular}{|c|c|c|c|c|c|c|c|c|c|c|c|}
\hline$P_{e}$ & 0.0 & 0.1 & 0.2 & 0.3 & 0.4 & 0.5 & 0.6 & 0.7 & 0.8 & 0.9 & 1.0 \\
\hline f01 & $9.92 \mathrm{E}-21$ & $2.54 \mathrm{E}-21$ & $4.14 \mathrm{E}-22$ & $5.06 \mathrm{E}-23$ & $4.74 \mathrm{E}-24$ & $2.90 \mathrm{E}-25$ & $9.43 \mathrm{E}-27$ & $1.21 \mathrm{E}-28$ & $8.48 \mathrm{E}-31$ & $1.18 \mathrm{E}-32$ & $5.57 \mathrm{E}+01$ \\
\hline f02 & 2.15E-18 & $7.80 \mathrm{E}-18$ & $2.31 \mathrm{E}-17$ & $4.78 \mathrm{E}-17$ & 7.03E-17 & $7.82 \mathrm{E}-17$ & $4.10 \mathrm{E}-17$ & 1.17E-17 & $1.85 \mathrm{E}-18$ & $8.89 \mathrm{E}-20$ & $2.13 \mathrm{E}+00$ \\
\hline f03 & $6.32 \mathrm{E}+02$ & 7.09E-10 & 7.13E-18 & $1.03 \mathrm{E}-22$ & $1.09 \mathrm{E}-24$ & $1.53 \mathrm{E}-23$ & $1.32 \mathrm{E}-20$ & $6.73 \mathrm{E}-16$ & 1.42E-09 & $2.00 \mathrm{E}-06$ & $5.47 \mathrm{E}+01$ \\
\hline f04 & $1.71 \mathrm{E}-07$ & $8.25 \mathrm{E}-09$ & 3.11E-10 & $8.83 \mathrm{E}-12$ & 2.03E-13 & $2.14 \mathrm{E}-15$ & $1.68 \mathrm{E}-17$ & 4.60E-13 & 7.45E-06 & $1.61 \mathrm{E}-02$ & $2.11 \mathrm{E}+00$ \\
\hline f05 & $1.80 \mathrm{E}+01$ & $1.62 \mathrm{E}+01$ & $1.21 \mathrm{E}+01$ & $2.46 \mathrm{E}+00$ & $6.75 \mathrm{E}-01$ & $2.14 \mathrm{E}-01$ & $1.35 \mathrm{E}+00$ & $6.81 \mathrm{E}+00$ & $1.86 \mathrm{E}+01$ & $2.36 \mathrm{E}+01$ & $5.45 \mathrm{E}+02$ \\
\hline f06 & $0.00 \mathrm{E}+00$ & $0.00 \mathrm{E}+00$ & $0.00 \mathrm{E}+00$ & $0.00 \mathrm{E}+00$ & $0.00 \mathrm{E}+00$ & $0.00 \mathrm{E}+00$ & $0.00 \mathrm{E}+00$ & $0.00 \mathrm{E}+00$ & $0.00 \mathrm{E}+00$ & $0.00 \mathrm{E}+00$ & $3.63 \mathrm{E}+01$ \\
\hline f07 & $6.05 \mathrm{E}-03$ & $5.29 \mathrm{E}-03$ & $4.28 \mathrm{E}-03$ & $3.48 \mathrm{E}-03$ & 3.23E- 03 & $2.66 \mathrm{E}-03$ & $2.29 \mathrm{E}-03$ & $2.20 \mathrm{E}-03$ & $1.87 \mathrm{E}-03$ & $1.71 \mathrm{E}-03$ & $2.50 \mathrm{E}-03$ \\
\hline f08 & $1.34 \mathrm{E}-02$ & $1.34 \mathrm{E}-02$ & $1.34 \mathrm{E}-02$ & $1.34 \mathrm{E}-02$ & $1.34 \mathrm{E}-02$ & $1.34 \mathrm{E}-02$ & $1.34 \mathrm{E}-02$ & $1.34 \mathrm{E}-02$ & $1.34 \mathrm{E}-02$ & $1.34 \mathrm{E}-02$ & $3.89 \mathrm{E}+03$ \\
\hline f09 & $0.00 \mathrm{E}+00$ & $0.00 \mathrm{E}+00$ & $0.00 \mathrm{E}+00$ & $5.39 \mathrm{E}-15$ & 2.03E-09 & $2.27 \mathrm{E}-01$ & $4.65 \mathrm{E}+00$ & $1.05 \mathrm{E}+01$ & $1.75 \mathrm{E}+01$ & $3.00 \mathrm{E}+01$ & $4.66 \mathrm{E}+01$ \\
\hline f10 & $2.26 \mathrm{E}-11$ & 1.19E-11 & $5.85 \mathrm{E}-12$ & 2.24E-12 & 7.54E-13 & $2.17 \mathrm{E}-13$ & 4.00E-14 & $1.01 \mathrm{E}-14$ & $6.16 \mathrm{E}-15$ & 5.57E-15 & $1.98 \mathrm{E}+00$ \\
\hline f11 & $0.00 \mathrm{E}+00$ & $0.00 \mathrm{E}+00$ & $0.00 \mathrm{E}+00$ & $0.00 \mathrm{E}+00$ & 2.47E-04 & $0.00 \mathrm{E}+00$ & $0.00 \mathrm{E}+00$ & $0.00 \mathrm{E}+00$ & $0.00 \mathrm{E}+00$ & $0.00 \mathrm{E}+00$ & $1.43 \mathrm{E}+00$ \\
\hline f12 & 2.60E-21 & $6.57 \mathrm{E}-22$ & $1.70 \mathrm{E}-22$ & $2.35 \mathrm{E}-23$ & $3.77 \mathrm{E}-24$ & $2.95 \mathrm{E}-25$ & $8.45 \mathrm{E}-27$ & $1.04 \mathrm{E}-28$ & $2.59 \mathrm{E}-30$ & $9.04 \mathrm{E}-31$ & $1.84 \mathrm{E}-01$ \\
\hline $\mathrm{f} 13$ & $1.43 E-30$ & $3.59 \mathrm{E}-21$ & $6.21 \mathrm{E}-22$ & $9.21 \mathrm{E}-23$ & 7.47E-24 & 4.99E-25 & $2.04 \mathrm{E}-26$ & $4.86 \mathrm{E}-28$ & 2.22E-29 & $1.04 \mathrm{E}-29$ & $1.48 \mathrm{E}+00$ \\
\hline f14 & $5.97 \mathrm{E}-08$ & $5.97 \mathrm{E}-08$ & $5.97 \mathrm{E}-08$ & $5.97 \mathrm{E}-08$ & $1.11 \mathrm{E}-02$ & $5.97 \mathrm{E}-08$ & $5.98 \mathrm{E}-08$ & $1.18 \mathrm{E}-07$ & 4.14E-04 & $1.24 \mathrm{E}-02$ & $1.18 \mathrm{E}-01$ \\
\hline f15 & $1.27 \mathrm{E}-05$ & $-9.20 \mathrm{E}-10$ & $-1.40 \mathrm{E}-08 *$ & $-1.40 \mathrm{E}-08$ & $-1.23 \mathrm{E}-08$ & $-1.40 \mathrm{E}-08$ & $2.39 \mathrm{E}-06$ & $-1.40 \mathrm{E}-08$ & $9.82 \mathrm{E}-07$ & $6.70 \mathrm{E}-06$ & $1.45 \mathrm{E}-05$ \\
\hline f16 & $5.16 \mathrm{E}-11$ & $9.94 \mathrm{E}-12$ & $5.53 \mathrm{E}-12$ & $6.21 \mathrm{E}-12$ & $5.54 \mathrm{E}-12$ & $1.64 \mathrm{E}-12$ & $2.26 \mathrm{E}-12$ & $2.31 \mathrm{E}-13$ & $2.81 \mathrm{E}-13$ & $6.67 \mathrm{E}-13$ & $1.32 \mathrm{E}-13$ \\
\hline f17 & 9.95E-16 & $2.85 \mathrm{E}-16$ & $1.67 \mathrm{E}-16$ & $1.67 \mathrm{E}-16$ & $1.67 \mathrm{E}-16$ & $1.67 \mathrm{E}-16$ & $1.67 \mathrm{E}-16$ & $1.67 \mathrm{E}-16$ & $1.67 \mathrm{E}-16$ & $1.67 \mathrm{E}-16$ & $1.67 \mathrm{E}-16$ \\
\hline f18 & $2.30 \mathrm{E}-14$ & 1.40E-14 & $1.35 \mathrm{E}-14$ & $1.26 \mathrm{E}-14$ & $9.56 \mathrm{E}-15$ & $7.18 \mathrm{E}-15$ & $6.50 \mathrm{E}-15$ & $5.88 \mathrm{E}-15$ & $3.57 \mathrm{E}-15$ & $2.98 \mathrm{E}-15$ & $3.46 \mathrm{E}-15$ \\
\hline f19 & $2.54 \mathrm{E}-07$ & 2.54E-07 & 2.54E-07 & $2.54 \mathrm{E}-07$ & 2.54E-07 & $2.54 \mathrm{E}-07$ & $2.54 \mathrm{E}-07$ & $2.54 \mathrm{E}-07$ & 2.54E-07 & $2.54 \mathrm{E}-07$ & $2.54 \mathrm{E}-07$ \\
\hline $\mathrm{f} 20$ & $1.59 \mathrm{E}-02$ & 3.96E-03 & 3.96E-03 & 7.73E-13 & $1.46 \mathrm{E}-13$ & $5.03 \mathrm{E}-14$ & 3.30E-14 & 3.96E-03 & $3.80 \mathrm{E}-14$ & $3.96 \mathrm{E}-03$ & $3.13 \mathrm{E}-13$ \\
\hline$f 21$ & $6.69 \mathrm{E}-01$ & $1.74 \mathrm{E}-01$ & 3.27E- 05 & 7.01E-06 & $1.27 \mathrm{E}-06$ & 4.96E-07 & $3.39 \mathrm{E}-07$ & $1.56 \mathrm{E}-07$ & 7.87E- 08 & $3.97 \mathrm{E}-08$ & $1.65 \mathrm{E}-08$ \\
\hline $\mathrm{f} 22$ & 3.03E-04 & $1.36 \mathrm{E}-05$ & 1.07E-05 & 4.79E- 07 & 2.73E- 07 & $1.32 \mathrm{E}-07$ & $8.55 \mathrm{E}-08$ & 3.61E-08 & 3.43E-08 & $1.31 \mathrm{E}-08$ & $2.11 \mathrm{E}-08$ \\
\hline $\mathrm{f} 23$ & $1.89 \mathrm{E}-05$ & $8.78 \mathrm{E}-06$ & $-8.50 \mathrm{E}-06$ & $-9.37 \mathrm{E}-06$ & $-9.64 \mathrm{E}-06$ & $-9.68 \mathrm{E}-06$ & $-9.30 \mathrm{E}-06$ & $-9.74 \mathrm{E}-06$ & $-9.75 \mathrm{E}-06$ & $-9.80 \mathrm{E}-06$ & $-9.80 \mathrm{E}-06$ \\
\hline F01 & $0.00 \mathrm{E}+00$ & $0.00 \mathrm{E}+00$ & $0.00 \mathrm{E}+00$ & $0.00 \mathrm{E}+00$ & $0.00 \mathrm{E}+00$ & $0.00 \mathrm{E}+00$ & $0.00 \mathrm{E}+00$ & $0.00 \mathrm{E}+00$ & $0.00 \mathrm{E}+00$ & $9.14 \mathrm{E}-29$ & $7.51 \mathrm{E}+03$ \\
\hline F02 & $7.30 \mathrm{E}+02$ & $3.87 \mathrm{E}-04$ & 1.13E- 08 & $1.06 \mathrm{E}-11$ & $1.84 \mathrm{E}-12$ & $3.19 \mathrm{E}-12$ & $1.58 \mathrm{E}-10$ & $1.22 \mathrm{E}-08$ & $1.37 \mathrm{E}-05$ & $4.63 \mathrm{E}-02$ & $4.68 \mathrm{E}+03$ \\
\hline F03 & $1.76 \mathrm{E}+07$ & $1.56 \mathrm{E}+05$ & $1.53 \mathrm{E}+05$ & $2.14 \mathrm{E}+05$ & $2.55 \mathrm{E}+05$ & $3.06 \mathrm{E}+05$ & $4.19 \mathrm{E}+05$ & $4.44 \mathrm{E}+05$ & $4.45 \mathrm{E}+05$ & $6.48 \mathrm{E}+05$ & $3.61 \mathrm{E}+06$ \\
\hline F04 & $2.37 \mathrm{E}+03$ & 3.37E-01 & $6.39 \mathrm{E}-04$ & 4.61E-05 & $6.58 \mathrm{E}-05$ & $1.02 \mathrm{E}-04$ & $3.71 \mathrm{E}-04$ & $1.84 \mathrm{E}-03$ & $1.24 \mathrm{E}-01$ & $5.71 \mathrm{E}+00$ & $3.57 \mathrm{E}+03$ \\
\hline F05 & $5.01 \mathrm{E}+02$ & $2.64 \mathrm{E}+01$ & $2.43 \mathrm{E}+00$ & $6.64 \mathrm{E}-01$ & $7.68 \mathrm{E}+00$ & $1.15 \mathrm{E}+02$ & $1.44 \mathrm{E}+02$ & $2.16 \mathrm{E}+02$ & $2.99 \mathrm{E}+02$ & $9.36 \mathrm{E}+02$ & $7.55 \mathrm{E}+03$ \\
\hline F06 & $2.37 \mathrm{E}+01$ & $2.08 \mathrm{E}+01$ & $1.82 \mathrm{E}+01$ & $1.37 \mathrm{E}+01$ & $9.18 \mathrm{E}+00$ & $8.80 \mathrm{E}+00$ & $1.05 \mathrm{E}+01$ & $1.80 \mathrm{E}+01$ & $2.30 \mathrm{E}+01$ & $3.56 \mathrm{E}+01$ & $2.11 \mathrm{E}+08$ \\
\hline F07 & $6.57 \mathrm{E}-04$ & $5.22 \mathrm{E}-11$ & $1.48 \mathrm{E}-17$ & $0.00 \mathrm{E}+00$ & $0.00 \mathrm{E}+00$ & $0.00 \mathrm{E}+00$ & $0.00 \mathrm{E}+00$ & 2.47E-04 & $1.73 \mathrm{E}-03$ & $1.05 \mathrm{E}-02$ & $5.76 \mathrm{E}+03$ \\
\hline F08 & $2.09 \mathrm{E}+01$ & $2.09 \mathrm{E}+01$ & $2.09 \mathrm{E}+01$ & $2.09 \mathrm{E}+01$ & $2.09 \mathrm{E}+01$ & $2.09 \mathrm{E}+01$ & $2.09 \mathrm{E}+01$ & $2.09 \mathrm{E}+01$ & $2.09 \mathrm{E}+01$ & $2.09 \mathrm{E}+01$ & $2.09 \mathrm{E}+01$ \\
\hline F09 & $0.00 \mathrm{E}+00$ & $0.00 \mathrm{E}+00$ & $0.00 \mathrm{E}+00$ & $0.00 \mathrm{E}+00$ & $1.42 \mathrm{E}-15$ & $5.71 \mathrm{E}-06$ & $1.76 \mathrm{E}+00$ & $7.74 \mathrm{E}+00$ & $1.51 \mathrm{E}+01$ & $2.74 \mathrm{E}+01$ & $6.79 \mathrm{E}+01$ \\
\hline F10 & $8.09 \mathrm{E}+01$ & $7.63 \mathrm{E}+01$ & $7.66 \mathrm{E}+01$ & $7.11 \mathrm{E}+01$ & $7.26 \mathrm{E}+01$ & $6.95 \mathrm{E}+01$ & $6.89 \mathrm{E}+01$ & $6.42 \mathrm{E}+01$ & $6.09 \mathrm{E}+01$ & $6.24 \mathrm{E}+01$ & $9.70 \mathrm{E}+01$ \\
\hline F11 & $3.07 \mathrm{E}+01$ & $2.97 \mathrm{E}+01$ & $2.98 \mathrm{E}+01$ & $2.97 \mathrm{E}+01$ & $2.95 \mathrm{E}+01$ & $2.93 \mathrm{E}+01$ & $2.84 \mathrm{E}+01$ & $2.91 \mathrm{E}+01$ & $2.86 \mathrm{E}+01$ & $2.87 \mathrm{E}+01$ & $2.88 \mathrm{E}+01$ \\
\hline F12 & $2.47 \mathrm{E}+04$ & $2.58 \mathrm{E}+04$ & $2.11 \mathrm{E}+04$ & $2.26 \mathrm{E}+04$ & $8.80 \mathrm{E}+03$ & $5.41 \mathrm{E}+03$ & $1.92 \mathrm{E}+03$ & $1.68 \mathrm{E}+03$ & $2.31 \mathrm{E}+03$ & $2.33 \mathrm{E}+03$ & $1.48 \mathrm{E}+04$ \\
\hline F13 & $2.58 \mathrm{E}+00$ & $2.70 \mathrm{E}+00$ & $2.83 \mathrm{E}+00$ & $2.98 \mathrm{E}+00$ & $3.19 \mathrm{E}+00$ & $3.37 \mathrm{E}+00$ & $3.52 \mathrm{E}+00$ & $3.87 \mathrm{E}+00$ & $4.58 \mathrm{E}+00$ & $5.55 \mathrm{E}+00$ & $7.32 \mathrm{E}+00$ \\
\hline F14 & $1.29 \mathrm{E}+01$ & $1.29 \mathrm{E}+01$ & $1.29 \mathrm{E}+01$ & $1.29 \mathrm{E}+01$ & $1.28 \mathrm{E}+01$ & $1.28 \mathrm{E}+01$ & $1.27 \mathrm{E}+01$ & $1.27 \mathrm{E}+01$ & $1.27 \mathrm{E}+01$ & $1.26 \mathrm{E}+01$ & $1.25 \mathrm{E}+01$ \\
\hline
\end{tabular}

The mean error is recorded based on 30 independent runs, and the best results are highlighted in boldface.

* A negative value means that the achieved value is better than the best result provided in Table 1 . 
Table A-5. Average ranking of CMM-BBO algorithms with different $P_{e}$ according to the Friedman test

\begin{tabular}{|c|c|c|c|c|c|c|c|c|c|c|c|c|}
\hline & $P_{e}$ & 0.0 & 0.1 & 0.2 & 0.3 & 0.4 & 0.5 & 0.6 & 0.7 & 0.8 & 0.9 & 1.0 \\
\hline \multirow[t]{2}{*}{ CMM-rcBBO } & Average ranking & 10.26 & 7.69 & 6.20 & 5.43 & 4.93 & 4.80 & 4.96 & 4.16 & 5.11 & 5.46 & 7.00 \\
\hline & Final rank & 11 & 10 & 8 & 6 & 3 & 2 & 4 & 1 & 5 & 7 & 9 \\
\hline \multirow[t]{2}{*}{ CMM-rcBBOg } & Average ranking & 10.23 & 7.27 & 5.58 & 4.95 & 4.50 & 4.49 & 5.34 & 4.70 & 5.46 & 6.08 & 7.41 \\
\hline & Final rank & 11 & 9 & 7 & 4 & 2 & 1 & 5 & 3 & 6 & 8 & 10 \\
\hline \multirow[t]{2}{*}{ CMM-pBBO } & Average ranking & 8.92 & 6.14 & 5.61 & 5.34 & 4.81 & 4.46 & 5.11 & 5.12 & 5.66 & 6.62 & 8.22 \\
\hline & Final rank & 11 & 8 & 6 & 5 & 2 & 1 & 3 & 4 & 7 & 9 & 10 \\
\hline \multirow[t]{2}{*}{ CMM-DE/BBO } & Average ranking & 7.54 & 6.15 & 5.72 & 5.19 & 5.22 & 4.96 & 5.01 & 5.34 & 5.82 & 6.45 & 8.61 \\
\hline & Final rank & 10 & 8 & 6 & 3 & 4 & 1 & 2 & 5 & 7 & 9 & 11 \\
\hline \multirow[t]{2}{*}{ Sum } & Average ranking & 36.95 & 27.24 & 23.11 & 20.91 & 19.46 & 18.70 & 20.42 & 19.32 & 22.05 & 24.61 & 31.23 \\
\hline & Final rank & 11 & 9 & 7 & 5 & 3 & 1 & 4 & 2 & 6 & 8 & 10 \\
\hline
\end{tabular}

